\title{
Donor Substituted Sulfonyl Carbenes, 21: Organothio Sulfonyl Carbenes
}

Kurt Schank $^{\mathrm{a}}$, Aboel-Magd A. Abdel Wahab ${ }^{\mathrm{b}}$, Stephan Bügler ${ }^{\mathrm{a}}$, Peter Eigen ${ }^{\mathrm{a}}$, Jürgen Jager ${ }^{\mathrm{a}}$ and Klaus Jost ${ }^{\mathrm{a}}$.

a) Department of Organic Chemistry, University of Saarbrücken, D-66041 Saarbrücken

b) Chemistry Department, Faculty of Science, University of Assiut, Assiut, Egypt

Key Words: Electrophilic organothio sulfonyl carbenes, generation and reactions with suitable nucleophiles/ $\alpha, \alpha$-disulfonyl thioethers, sulfonyl cyclopropanes, sulfonyl pyrazoles, carbene dimers; sulfinate - sulfone Pummerer rearrangement.

\begin{abstract}
Organothio sulfonyl carbenes $\underline{\mathbf{3}}$ have been generated via ylid thermolysis or via $\alpha$-elimination starting from $\alpha$-chloro $\alpha$-organothio sulfones and their derivatives. They have been captured by suitable nucleophilic trapping reagents (diazomethane, enol ethers, and others). Their nucleophilic carbenoid precursors could be trapped by an electrophilic olefin (ketene dithioacetal S,S-dioxides as Michael acceptors). Stable carbene Z-dimers could be obtained under various conditions. Bromine catalyzed isomerization to E-isomers proved to be reversible.
\end{abstract}

\section{INTRODUCTION}

During the sixties the pioneering work of U. Schöllkopf et al. ${ }^{2-5}$ opened the door to the study of the chemistry of monothiocarbenes ${ }^{3}$, chloro thiocarbenes ${ }^{4}$ as well as dithiocarbenes ${ }^{5}$. Following on our studies on $\alpha$-sulfonyl ethers ${ }^{6}$ we investigated a.o. the typical reactions of donor substituted sulfonyl carbenes (donors: methoxy $^{1}$, organothio ${ }^{7}$ ) in order to classify their philicity ${ }^{8}$. Decomposition of ylids $\underline{\mathbf{1}}$ (method A) or $\alpha$-eliminations of $\mathrm{YZ}$ (predominantly $\mathrm{HCl}$ ) (method B) from suitable precursors like $\underline{4}$ were preferentially used for carbene generation (Scheme 1).

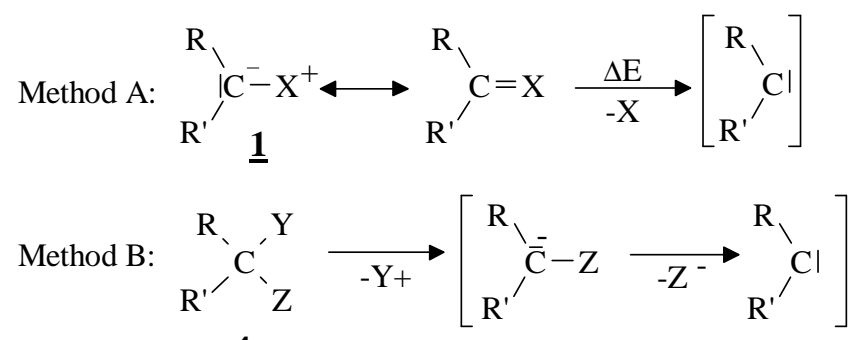

$\underline{4}$

Scheme 1: Usual carbene generation pathways

\section{CARBENES FROM YLID-PRECURSORS, METHOD A.}

Ylids $\underline{1}$ prepared from corresponding active methylene compounds $\underline{\mathbf{2}}$ should give rise to corresponding carbenes $\underline{\mathbf{3}}$ (Formula 1).<smiles>[R]SC([X])S([R])=O</smiles>

\begin{tabular}{cl|ccc} 
Ylids: & $\underline{\mathbf{1}}$ & $\mathrm{X}$ & $\mathrm{R}^{1}$ & $\mathrm{R}^{2}$ \\
\hline & $\underline{\mathbf{a}}^{11}$ & $\mathrm{~N}_{2}$ & $4-\mathrm{Cl}_{-}-\mathrm{C}_{6} \mathrm{H}_{4}$ & $\mathrm{CH}_{3}$ \\
& $\underline{\mathrm{IC}}_{6} \mathrm{H}_{5}$ & $\mathrm{C}_{6} \mathrm{H}_{5}$ & $\mathrm{C}_{6} \mathrm{H}_{5}$ \\
& $\underline{\mathbf{c}}$ & $\mathrm{S}\left(\mathrm{CH}_{3}\right)_{2}$ & $4-\mathrm{CH}_{3}-\mathrm{C}_{6} \mathrm{H}_{4}$ & $\mathrm{CH}_{3}$ \\
& $\underline{\mathbf{d}}$ & $\mathrm{S}\left(\mathrm{CH}_{3}\right)_{2}$ & $4-\mathrm{Cl}^{2} \mathrm{C}_{6} \mathrm{H}_{4}$ & $\mathrm{CH}_{3}$
\end{tabular}




$$
\begin{aligned}
& \mathrm{R}^{1} \mathrm{SO}_{2}^{\mathrm{R}^{2} \mathrm{~S}}{ }_{2}^{\longrightarrow} \mathrm{CH}_{2} \\
& \mathrm{R}^{1} \mathrm{SO}_{2}^{\mathrm{R}^{2} \mathrm{~S}}{ }_{\underline{\mathbf{3}}}^{\mathrm{Cl}} \\
& \mathrm{R}^{1} \mathrm{SO}_{2}^{2} \overbrace{\mathrm{Cl}}^{\mathrm{C}} \\
& 4
\end{aligned}
$$

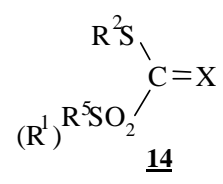

Formula 1: Carbenes $\underline{\mathbf{3}}$, precursors $\underline{\mathbf{1}}, \underline{\mathbf{2}}, \underline{\mathbf{4}}$ and methylenation products $\underline{\mathbf{1 4}}$

Attempts to generate $\underline{\mathbf{1 a}}^{9}$ via diazo transfer from tosyl azide ${ }^{10}$ proved to be unsuccessful and only unchanged starting materials were recovered. $1 \mathbf{b}$ has been reported to decompose in situ at $-10^{\circ} \mathrm{C}$ yielding carbon dioxide and diphenyl disulfide ${ }^{11}$. After a series of unsuccessful attempts sulfonium ylids $\underline{\mathbf{1}} \underline{\mathbf{c}}, \underline{\mathbf{d}}$ could be obtained in poor yields as rather stable compounds by use of dimethyl $\mathrm{N}$-succinimidosulfonium chloride $\underline{\mathbf{5}}^{12}$ (Scheme 2).

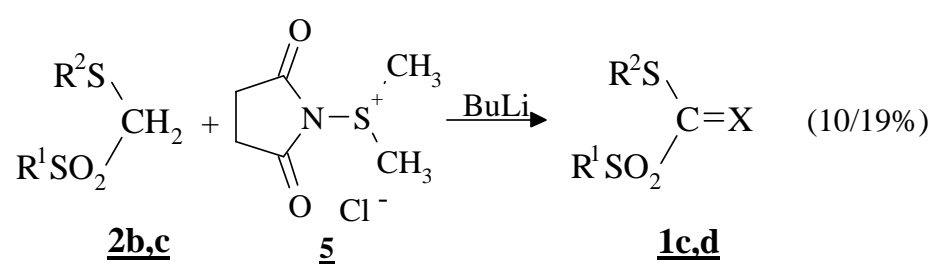

Scheme 2: Synthesis of dimethyl sulfonium arylsulfonyl methylthio methylids $\underline{\mathbf{1 c}}, \underline{\mathbf{d}}$

Thermal decomposition of $\underline{\mathbf{1 d}}$ (method A) in a boiling Z,E mixture (3:1) of 1,2-dimethoxyethylene (우) (excess) gave cyclopropane $\underline{\mathbf{7}}$ and various other compounds determined by ${ }^{1} \mathrm{H}-\mathrm{NMR}$ analysis and comparison with independently prepared material ${ }^{12 a}($ Scheme 3$)$.

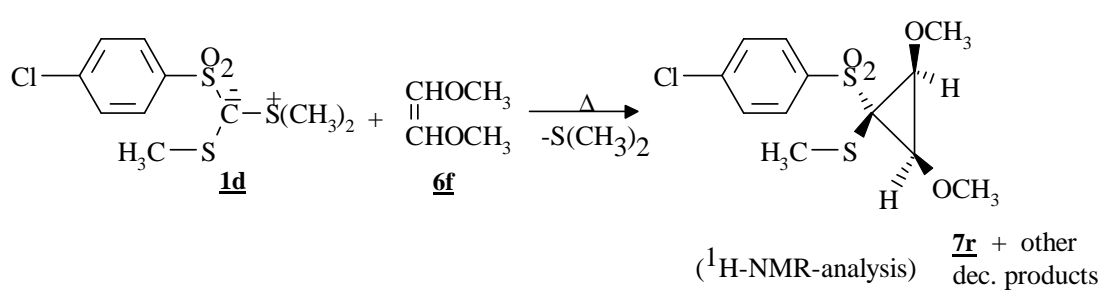

Scheme 3: Trapping of carbene $\underline{\mathbf{3 c}}$ by means of $\underline{\mathbf{6 f}}$

\section{CARBENES FROM CARBANIONIC PRECURSORS, METHOD B}

No further attempts were made using method A because yields of $\underline{7}$ were too low and because parallel experiments according to method B proved to be more successful. Subsequently dithioacetal-S,S-dioxides $\underline{\mathbf{2}}$ 
have been prepared according to known procedures $\left(\underline{\mathbf{2 d}}^{11}\right.$ was not utilized in this paper; other preparative methods ${ }^{13}$ of starting compounds $\underline{2}$ were not applied):

Method I ("Gibson's method") ${ }^{14}: \alpha$-Sulfenylation of $\alpha$-sulfonyl ketones followed by acyl cleavage;

Method II: Selective unsymmetrical bisoxidation of dithioacetals ${ }^{15}$;

Method IIIa: Nucleophilic $\alpha$-substitution of negatively substituted alkyl thioethers by means of sulfinate anion ${ }^{16}$;

Method IIIb: Nucleophilic $\alpha$-substitution of chloride in chloromethyl sulfones by means of thiolate anion ${ }^{17}$;

Method IV: "sulfinate-sulfone Pummerer rearrangement"18 during sulfinylation of methylene activated compounds with sulfinyl chlorides ("Philbin-Wheeler method") ${ }^{19}$ followed by twofold acyl cleavage ${ }^{20}$ (Scheme 4).

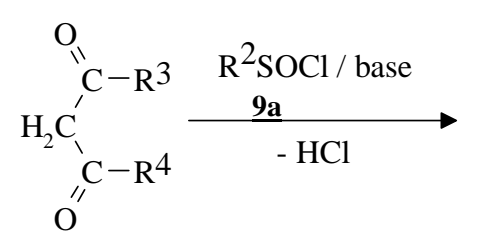

$\underline{\mathbf{8}}$

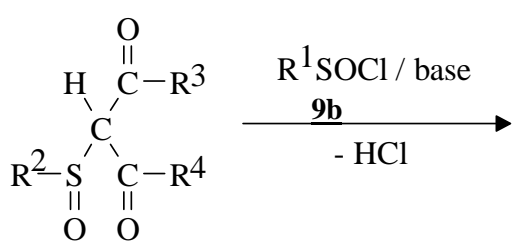

$\underline{10}$

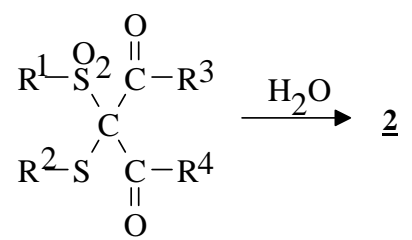

$\underline{11}$

Scheme 4: Synthesis of $\alpha$-sulfonyl thioethers $\underline{\mathbf{2}}$ (organothiomethyl sulfones) via "sulfinate-sulfone Pummerer rearrangement"

Although intermediates $\underline{\mathbf{1 0}}$ and $\underline{\mathbf{1 1}}$ can be isolated, however, the use of $\underline{\mathbf{9 a}}, \underline{\mathbf{9 b}}\left(\mathrm{R}^{1}=\mathrm{R}^{2}\right)$ afforded $\underline{\mathbf{2}}$ from $\underline{\mathbf{8}}$ via a one-pot procedure.

Monochlorination of $\underline{\mathbf{2}}$ to give corresponding $\alpha$-chloro $\alpha$-sulfonyl thioethers $\underline{4}$ by means of sulfuryl chloride ensued easily $^{21}$, although caution was needed with some examples to prevent undesired dichlorination ${ }^{12 a}$ (formation of 12). Stepwise halogenation of $\underline{\mathbf{2}}$ with sulfuryl chloride and finally with bromine-pyridine 1:1 mixture in chloroform gave rise to crystalline $\alpha$-bromo- $\alpha$-chloro- $\alpha$-sulfonyl thioethers $\underline{13}$. Methine proton exchange in $\mathbf{4}$ (or halogen exchange in 12,13 under PTC-conditions) led to reactive carbanions $\underline{4}^{-}$which could be trapped by electrophilic ketene dithioacetal-S,S-dioxide $\underline{14}$ via a Michael-type addition at low temperature and short reaction times to give $\gamma$-disulfones $\underline{15}$ (Scheme 5).

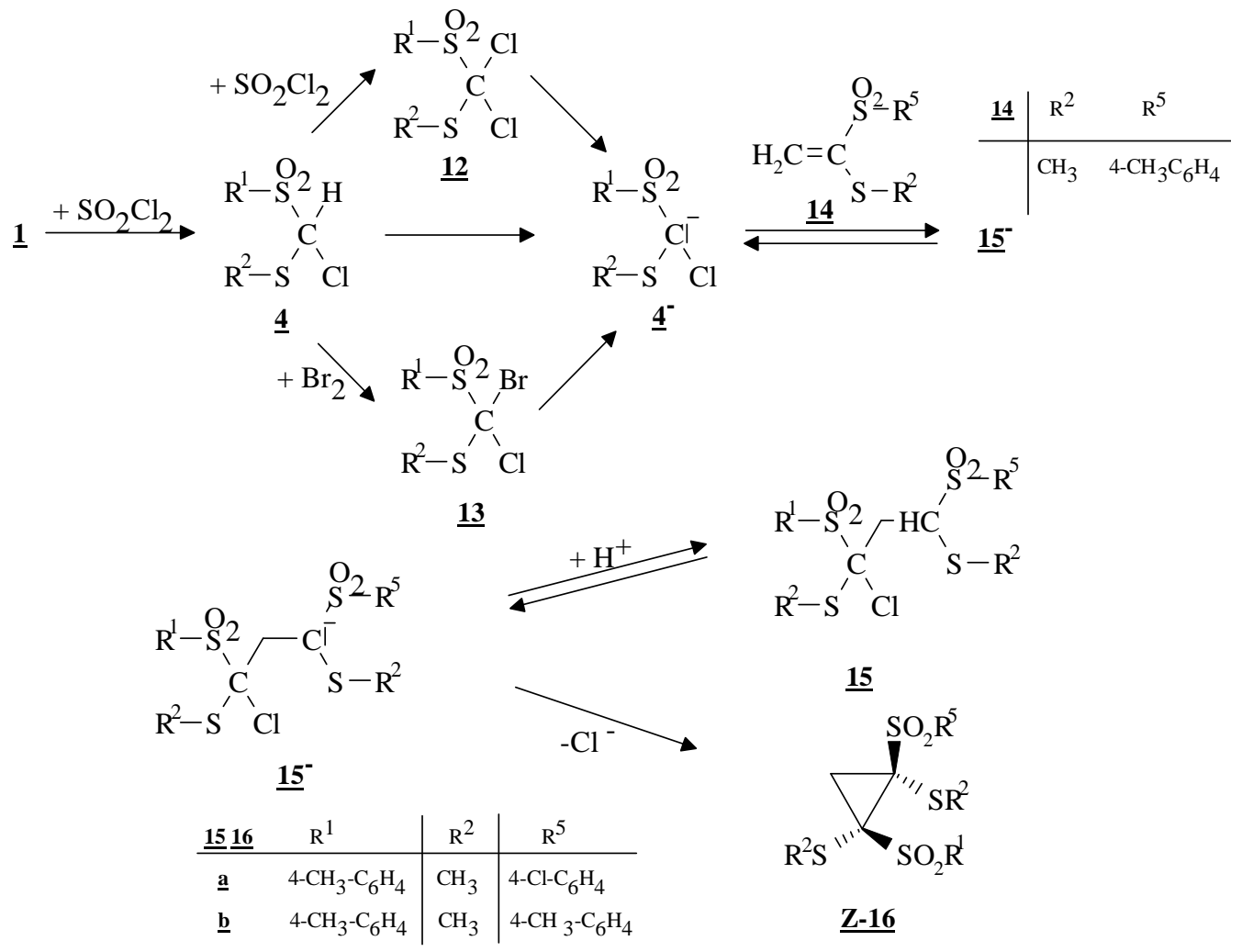


Although $\underline{15}$ was shown by ${ }^{1} \mathrm{H}-\mathrm{NMR}$ spectroscopy to be a mixture of diastereomers (m.d.), the Z-isomer $\underline{\mathbf{1 6}}$ could be obtained exclusively. This surprising result ${ }^{23 c}$ could be due to a fast retro-addition/addition equilibrium connected with steric equilibration. It is known that cyclopropanations of $\gamma$-halogen $\alpha$-sulfonyl carbanions presuppose an appropriate steric arrangement ${ }^{23}$ (Formula 2).

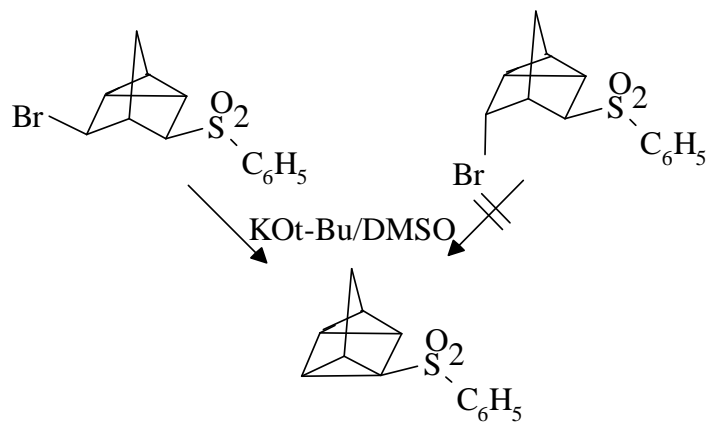

Formula 2: Stereospecific cyclopropanation

Whereas methoxy arylsulfonyl carbenes ${ }^{1}$ were trapped instantly by diazomethane to give rather inert ketene $\mathrm{O}, \mathrm{S}$-acetal S,S-dioxides, the corresponding ketene S,S-acetal S,S-dioxide $\underline{\mathbf{1 4}}$, generated in situ from title carbene $\underline{\mathbf{3 b}}$ and diazomethane, could be detected only by thin layer chromatography (TLC); owing to its high reactivity as a Michael acceptor (according to scheme 5) $\underline{\mathbf{4 b}}$ immediately underwent cyclopropanation yielding Z-16b (Scheme 6).

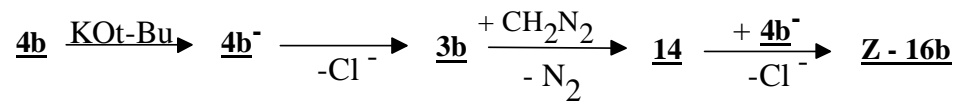

Scheme 6: Diazomethane trapping reaction of carbene $\underline{\mathbf{3 b}}$

The commonly used Michael acceptor ethyl acrylate added to the reaction mixture did not participate in these cyclopropanations, i.e. 14 was a superior Michael acceptor. Furthermore, Scheme 6 showed that diazomethane (excess) was much less nucleophilic toward 14 than was $\underline{\mathbf{4 b}}$. Nevertheless, independently prepared $\underline{\mathbf{1 4}}$ reacted with diazomethane via [3+2] cycloaddition to give pyrazoline $\underline{\mathbf{1 7 a}}$ which eliminated sulfinic acid to 18a after treatment with hydrochloric acid (Scheme 7).

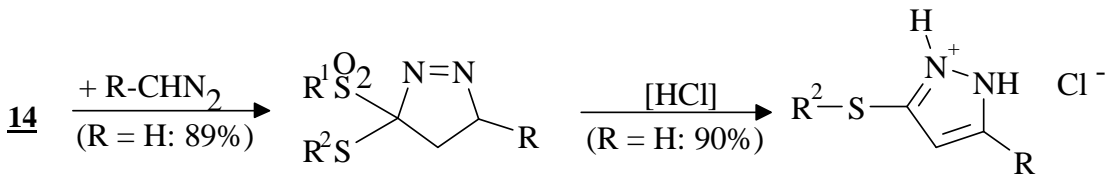

$$
\begin{aligned}
& 17 \\
& \underline{18} \\
& \begin{array}{lll|ccc}
\cline { 2 - 4 }\left(\mathrm{R}^{1}, \mathrm{R}^{2} \text { cf. } \underline{\mathbf{2}}, \underline{\mathbf{3 b}}\right) & \underline{\mathbf{a}} & \mathrm{H} & 4-\mathrm{CH}_{3}-\mathrm{C}_{6} \mathrm{H}_{4} & \mathrm{CH}_{3} \\
& \underline{\mathbf{b}} & \mathrm{CH}_{3} & 4-\mathrm{CH}_{3}-\mathrm{C}_{6} \mathrm{H}_{4} & \mathrm{CH}_{3}
\end{array}
\end{aligned}
$$

Scheme 7: 3-Methylthio pyrazolium salts $\underline{\mathbf{1 8}}$ via cycloaddition and heteroaromatization

A corresponding cycloaddition of diazoethane to 14 led immediately to formation of 5-methyl3-methylthio-pyrazole 18b (isolated as the hydrochloride). In this case the pyrazoline intermediate 17b could not be isolated. Consequently, reaction of carbene $\underline{\mathbf{3 b}}$ with two equivalents of diazoethane gave rise to 4,5-dimethyl-3-methylthio-pyrazole $\mathbf{2 1}$ (isolated as its hydrochloride in 77\% yield); surprisingly, a small amount (4\%) of 4,5-dimethyl-3- (4-tosyl)-pyrazole was formed additionally (Scheme 8). 


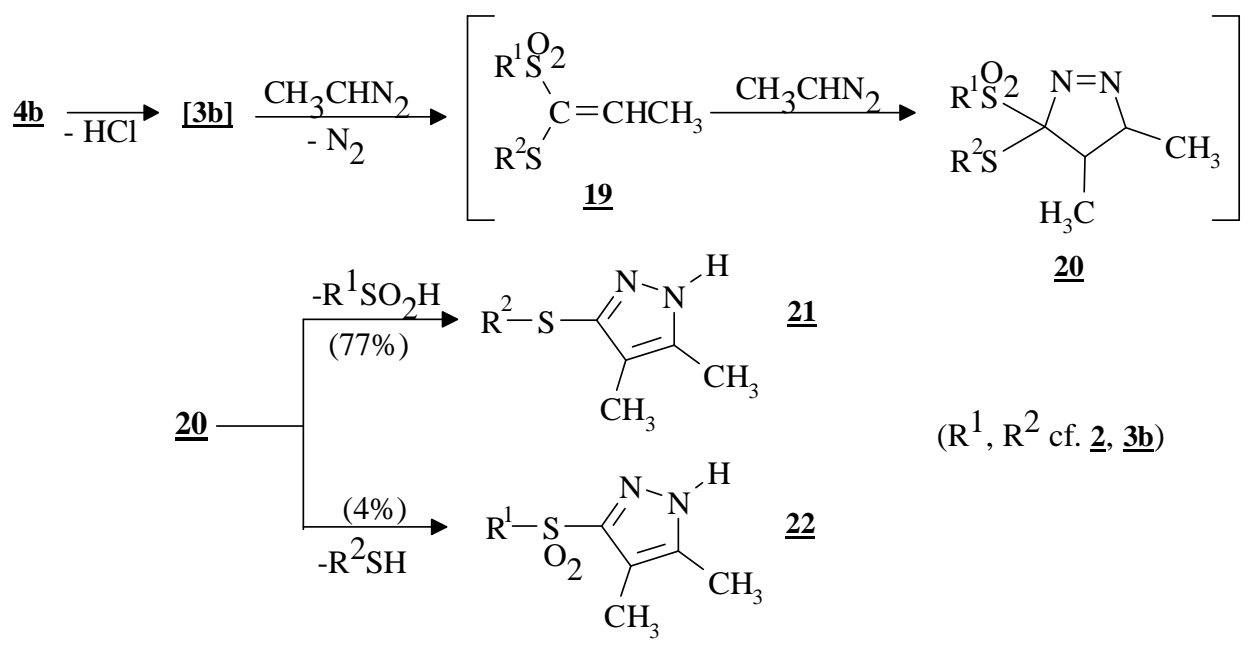

Scheme 8: Reaction of diazoethane (excess) with carbene $\underline{\mathbf{3 b}}$

\section{CYCLOPROPANES VIA TRAPPING REACTIONS OF THE TITLE CARBENES WITH REACTIVE OLEFINS}

Whereas trapping experiments of PTC-generated carbenes $\underline{\mathbf{3}}$ with ordinary olefins (i-butene, cyclohexene, E-stilbene, tetraphenylethylene, phenanthrene) failed, corresponding experiments with enol ethers, endiol ethers, and styrenes were successful. This selectivity classified carbenes $\underline{\mathbf{3}}$ as less reactive electrophilic carbenes. Nevertheless, in the absence of trapping olefins or in the presence of less reactive olefins carbenes $\underline{\mathbf{3}}$ suffered self-decomposition in presence of their carbenoids $\underline{4}^{-}$with formation of corresponding sulfinates $\underline{\mathbf{2 3}}$. As has been shown in earlier papers on arylsulfonyl methoxy carbenes ${ }^{24} \mathrm{~S}$-addition to sulfinate leads to $\beta$-disulfones in good yields. Principally, two pathways leading to formation of sulfinate seam to be possible: a) $\alpha$-elimination from carbenoids 4 ; b) $\beta$-elimination from carbene-carbenoid adducts, this last step according to the Stuffer-Backer mechanism ${ }^{25}$ (Scheme 9).

a) $\alpha$-eliminations:

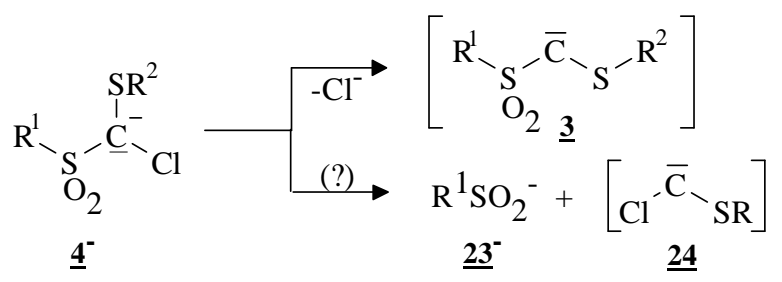

b) carbenoid-carbene C,C-connections with subsequent B-eliminations:

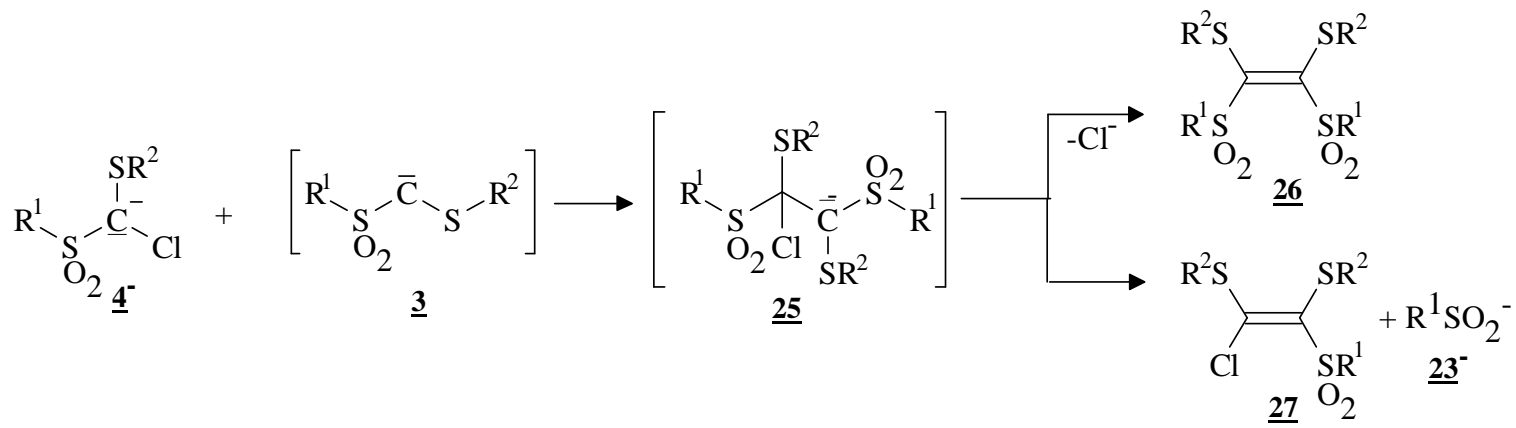


c) Trithioorthoformate $\mathrm{S}, \mathrm{S}, \mathrm{S}^{\prime}, \mathrm{S}^{\prime}$-tetroxides $(\underline{\mathbf{2 8}})$ via sulfinate $\mathrm{S}$-addition to carbenes:

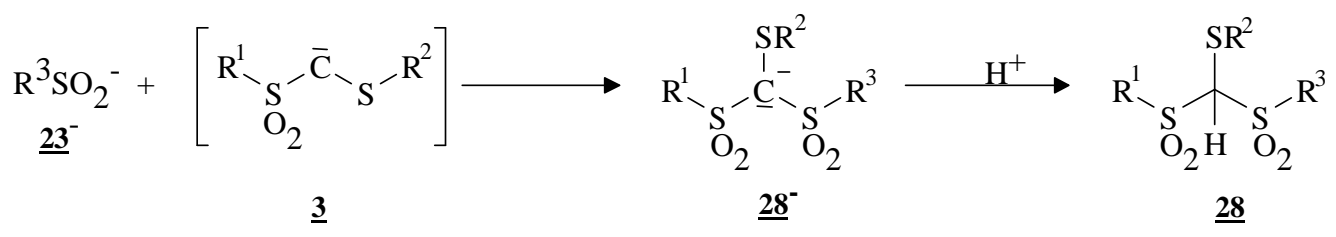

\begin{tabular}{c|ccc}
$\underline{\mathbf{2 8}}$ & $\mathrm{R}^{1}$ & $\mathrm{R}^{2}$ & $\mathrm{R}^{3}$ \\
\hline$\underline{\mathbf{a}}$ & $4-\mathrm{CH}_{3}-\mathrm{C}_{6} \mathrm{H}_{4}$ & $\mathrm{CH}_{3}$ & $4-\mathrm{CH}_{3}-\mathrm{C}_{6} \mathrm{H}_{4}$ \\
$\underline{\mathbf{b}}$ & $4-\mathrm{Cl}^{-\mathrm{C}_{6} \mathrm{H}_{4}}$ & $\mathrm{CH}_{3}$ & $4-\mathrm{CH}_{3}-\mathrm{C}_{6} \mathrm{H}_{4}$ \\
$\underline{\mathbf{c}}$ & $4-\mathrm{CH}_{3}-\mathrm{C}_{6} \mathrm{H}_{4}$ & $4-\mathrm{CH}_{3}-\mathrm{C}_{6} \mathrm{H}_{4}$ & $4-\mathrm{CH}_{3}-\mathrm{C}_{6} \mathrm{H}_{4}$
\end{tabular}

Scheme 9: Possibilities for generation and reaction of sulfinate during base catalyzed decomposition of $\underline{4}$

Sulfinate S-addition occurs only with donor-acceptor substituted carbenes ${ }^{1,26}$ whereas O-addition followed by fragmentation usually predominates ${ }^{27}$. This latter reaction would lead to disulfides $\underline{\mathbf{2 9}}$ and thiosulfonates $\underline{\mathbf{3 0}}$ which were actually identified together with up to ten by-products (TLC-analysis) (Formula 3).

$$
\mathrm{R}-\mathrm{S}-\mathrm{S}-\mathrm{R}^{1(3)} \quad \mathrm{R}-\underset{\mathrm{O}}{\mathrm{O}}-\mathrm{S}-\mathrm{R}^{1(3)}
$$

$\underline{29}$

$\underline{\mathbf{3 0}}$

Formula 3: Disulfides $\underline{\mathbf{2 9}}$ and Thiosulfonates $\underline{\mathbf{3 0}}$

However, the origin of the formation of $\underline{\mathbf{2 9}}, \underline{\mathbf{3 0}}$ has not been investigated because these compounds are usual decomposition products of the corresponding sulfinic acids $\underline{\mathbf{2 3}}$.

Generation of carbenes $\underline{\mathbf{3}}$ was carried out under PTC conditions $\left[\mathrm{KOH} / \mathrm{H}_{2} \mathrm{O} / \mathrm{CH}_{2} \mathrm{Cl}_{2}\right]$ using 18 -crown-6 as the most effective catalyst (found order of efficiency during cyclopropanations of 2,3-dihydrofuran $\underline{\mathbf{6 d}}$ with $\underline{\mathbf{3 b}}$ yielding 7h: 18 -crown-6 $(59 \%)>$ triethylamine $(49 \%)>$ tri-n-butylamine $(47 \%)>$ DBU $(1.8$-diazabicyclo [5.4.0.] undec-7-en) $(21 \%)>$ Hünig base (ethyldi-i-propylamine) $(13 \%)>\mathrm{N}, \mathrm{N}$-diethylaniline $(12 \%))$.

Use of aprotic reaction conditions (solvents: absol. THF or DMF; base: potassium t-butoxide) led to lower yields. The following reactive olefins $\underline{\mathbf{6}}$ have been utilized for cyclopropanations: Alkyl vinyl ethers $\underline{\mathbf{6}}-\underline{\mathbf{c}}$, cyclic enol ethers $\underline{\mathbf{6 d}}, \underline{\mathbf{e}}$, styrenes $\underline{\mathbf{6}}, \mathbf{g}, 1,2$-enediol ethers $\underline{\mathbf{6 h}}, \underline{\mathbf{6}}$ (Z and E) (Formula 4).

Olefins $\underline{6}$ : $\mathrm{CH}_{2}=\mathrm{CH}-\mathrm{O}-\mathrm{R}$

\begin{tabular}{l|l}
$\mathbf{6}$ & \multicolumn{1}{|c}{$\mathrm{R}$} \\
\hline$\underline{\mathbf{a}}$ & $\mathrm{CH}_{3}$ \\
$\underline{\mathbf{b}}$ & $\mathrm{C}_{2} \mathrm{H}_{5}$ \\
$\mathbf{c}$ & $\mathrm{n}-\mathrm{C}_{4} \mathrm{H}_{9}$
\end{tabular}<smiles>C[C@@H]1C=CO1</smiles>

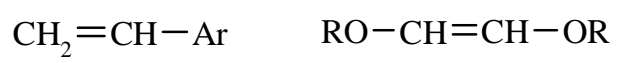

\begin{tabular}{l|l}
$\mathbf{6}$ & $\mathrm{n}$ \\
\hline$\underline{\mathbf{d}}$ & 2 \\
$\mathbf{e}$ & 3
\end{tabular}
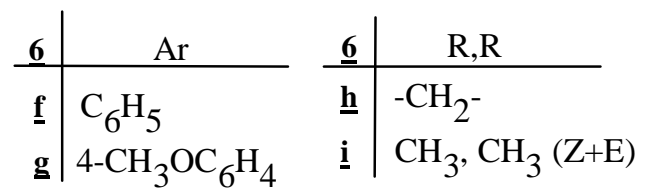
Table I: Cyclopropanes $\mathbf{7}$

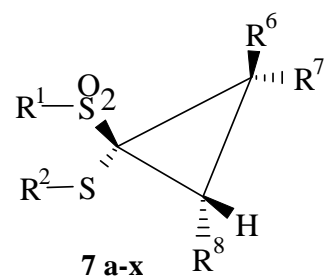

\begin{tabular}{|c|c|c|c|c|c|c|}
\hline$\underline{7}$ & $\mathrm{R}^{1}$ & $\mathrm{R}^{2}$ & $\mathrm{R}^{6}$ & $\mathrm{R}^{7}$ & $\mathrm{R}^{8}$ & yield $(\%)$ \\
\hline$\underline{\mathbf{a}}$ & $4-\mathrm{CH}_{3} \mathrm{C}_{6} \mathrm{H}_{4}$ & $\mathrm{CH}_{3}$ & $\mathrm{H}$ & $\mathrm{OCH}_{3}$ & $\mathrm{H}$ & 76.5 \\
\hline$\underline{\mathbf{b}}$ & 4- $\mathrm{ClC}_{6} \mathrm{H}_{4}$ & $\mathrm{CH}_{3}$ & $\mathrm{H}$ & $\mathrm{OCH}_{3}$ & $\mathrm{H}$ & 31.4 \\
\hline$\underline{\mathbf{c}}$ & $4-\mathrm{CH}_{3} \mathrm{C}_{6} \mathrm{H}_{4}$ & $\mathrm{CH}_{3}$ & $\mathrm{H}$ & $\mathrm{OC}_{2} \mathrm{H}_{5}$ & $\mathrm{H}$ & 86.6 \\
\hline$\underline{\mathbf{d}}$ & 4- $\mathrm{ClC}_{6} \mathrm{H}_{4}$ & $\mathrm{CH}_{3}$ & $\mathrm{H}$ & $\mathrm{OC}_{2} \mathrm{H}_{5}$ & $\mathrm{H}$ & 78.4 \\
\hline$\underline{\mathbf{e}}$ & $4-\mathrm{CH}_{3} \mathrm{C}_{6} \mathrm{H}_{4}$ & $\mathrm{CH}_{3}$ & $\mathrm{H}$ & $\mathrm{OC}_{4} \mathrm{H}_{9}$ & $\mathrm{H}$ & 63.6 \\
\hline$\underline{\mathbf{f}}$ & 4- $\mathrm{ClC}_{6} \mathrm{H}_{4}$ & $\mathrm{CH}_{3}$ & $\mathrm{H}$ & $\mathrm{OC}_{4} \mathrm{H}_{9}$ & $\mathrm{H}$ & 71.7 \\
\hline $\mathbf{g}$ & $\mathrm{C}_{6} \mathrm{H}_{5}$ & $\mathrm{CH}_{3}$ & $\mathrm{H}$ & \multicolumn{2}{|c|}{$-\mathrm{O}\left(\mathrm{CH}_{2}\right)_{2-}$} & $51.8(57.3)^{\mathrm{a}}$ \\
\hline$\underline{\mathbf{h}}$ & 4- $\mathrm{CH}_{3} \mathrm{C}_{6} \mathrm{H}_{4}$ & $\mathrm{CH}_{3}$ & $\mathrm{H}$ & \multicolumn{2}{|c|}{$-\mathrm{O}\left(\mathrm{CH}_{2}\right)_{2^{-}}$} & 59 \\
\hline$\underline{\mathbf{i}}$ & 4- $\mathrm{ClC}_{6} \mathrm{H}_{4}$ & $\mathrm{CH}_{3}$ & $\mathrm{H}$ & \multicolumn{2}{|c|}{$-\mathrm{O}\left(\mathrm{CH}_{2}\right)_{2-}$} & 37 \\
\hline $\mathbf{j}$ & $4-\mathrm{CH}_{3} \mathrm{C}_{6} \mathrm{H}_{4}$ & 4- $\mathrm{CH}_{3} \mathrm{C}_{6} \mathrm{H}_{4}$ & $\mathrm{H}$ & \multicolumn{2}{|c|}{$-\mathrm{O}\left(\mathrm{CH}_{2}\right)_{3^{-}}$} & 37.4 \\
\hline$\underline{\mathbf{k}}$ & $4-\mathrm{CH}_{3} \mathrm{C}_{6} \mathrm{H}_{4}$ & $\mathrm{CH}_{3}$ & $\mathrm{H}$ & $\mathrm{C}_{6} \mathrm{H}_{5}$ & $\mathrm{H}$ & 50 \\
\hline$\underline{\mathbf{1}}$ & $4-\mathrm{CH}_{3} \mathrm{C}_{6} \mathrm{H}_{4}$ & $\mathrm{CH}_{3}$ & $\mathrm{H}$ & $4-\mathrm{CH}_{3} \mathrm{OC}_{6} \mathrm{H}_{4}$ & $\mathrm{H}$ & 46 \\
\hline$\underline{\mathbf{m}}$ & 4- $\mathrm{CH}_{3} \mathrm{C}_{6} \mathrm{H}_{4}$ & 4- $\mathrm{CH}_{3} \mathrm{C}_{6} \mathrm{H}_{4}$ & $\mathrm{H}$ & $\mathrm{C}_{6} \mathrm{H}_{5}$ & $\mathrm{H}$ & 71.5 \\
\hline$\underline{\mathbf{n}}$ & 4- $\mathrm{CH}_{3} \mathrm{C}_{6} \mathrm{H}_{4}$ & 4- $\mathrm{CH}_{3} \mathrm{C}_{6} \mathrm{H}_{4}$ & $\mathrm{H}$ & 4- $\mathrm{CH}_{3} \mathrm{OC}_{6} \mathrm{H}_{4}$ & $\mathrm{H}$ & 66.6 \\
\hline$\underline{\mathbf{0}}$ & 4- $\mathrm{CH}_{3} \mathrm{C}_{6} \mathrm{H}_{4}$ & $\mathrm{CH}_{3}$ & $\mathrm{H}$ & \multicolumn{2}{|c|}{$-\mathrm{O}-\mathrm{CH}_{2}-\mathrm{O}-$} & 61.5 \\
\hline$\underline{\mathbf{p}}$ & $\mathrm{C}_{6} \mathrm{H}_{5}$ & $\mathrm{CH}_{3}$ & $\mathrm{H}$ & $\mathrm{OCH}_{3}$ & $\mathrm{OCH}_{3}$ & 36.2 \\
\hline$\underline{\mathbf{q}}$ & $4-\mathrm{CH}_{3} \mathrm{C}_{6} \mathrm{H}_{4}$ & $\mathrm{CH}_{3}$ & $\mathrm{H}$ & $\mathrm{OCH}_{3}$ & $\mathrm{OCH}_{3}$ & $87(91)$ \\
\hline$\underline{\mathbf{r}}$ & $4-\mathrm{ClC}_{6} \mathrm{H}_{4}$ & $\mathrm{CH}_{3}$ & $\mathrm{H}$ & $\mathrm{OCH}_{3}$ & $\mathrm{OCH}_{3}$ & 90.5 \\
\hline$\underline{\mathbf{s}}$ & 4- $\mathrm{CH}_{3} \mathrm{C}_{6} \mathrm{H}_{4}$ & $4-\mathrm{CH}_{3} \mathrm{C}_{6} \mathrm{H}_{4}$ & $\mathrm{H}$ & $\mathrm{OCH}_{3}$ & $\mathrm{OCH}_{3}$ & 56 \\
\hline$\underline{\mathbf{t}}$ & $4-\mathrm{CH}_{3} \mathrm{C}_{6} \mathrm{H}_{4}$ & $\mathrm{CH}_{3}$ & $\mathrm{OCH}_{3}$ & $\mathrm{H}$ & $\mathrm{OCH}_{3}$ & 10.3 \\
\hline$\underline{\mathbf{u}}$ & $4-\mathrm{CH}_{3} \mathrm{C}_{6} \mathrm{H}_{4}$ & 4- $\mathrm{CH}_{3} \mathrm{C}_{6} \mathrm{H}_{4}$ & $\mathrm{OCH}_{3}$ & $\mathrm{H}$ & $\mathrm{OCH}_{3}$ & 54 \\
\hline$\underline{\mathbf{v}}$ & \multicolumn{2}{|c|}{$-\left(\mathrm{CH}_{2}\right)_{2^{-}}$} & $\mathrm{H}$ & $\mathrm{OCH}_{3}$ & $\mathrm{OCH}_{3}$ & $66.9^{\mathrm{b}}$ \\
\hline$\underline{\mathbf{w}}$ & \multicolumn{2}{|c|}{$-\left(\mathrm{CH}_{2}\right)_{3^{-}}$} & $\mathrm{H}$ & $\mathrm{OCH}_{3}$ & $\mathrm{OCH}_{3}$ & $35.7^{\mathrm{b}}$ \\
\hline$\underline{\mathbf{x}}$ & \multicolumn{2}{|c|}{$\langle>$} & $\mathrm{H}$ & $\mathrm{OCH}_{3}$ & $\mathrm{OCH}_{3}$ & 51.6 \\
\hline
\end{tabular}

${ }^{\mathrm{a}}$ in the presence of $\mathrm{Me}_{3} \mathrm{SiCl} ;{ }^{\mathrm{b}} \mathrm{BuLi}$ in hexane

Preparation of cyclopropane $\underline{\mathbf{7 v}}$ needed the chloro compound $\underline{\mathbf{4 f}}$ derived from cyclic sulfone $\underline{\mathbf{2 f}}$. Since these simple heterocyclic compounds hitherto were unknown the method of preparation is given. 1,3-Dithiolane ${ }^{28}(\underline{\mathbf{3 1}})$ could be dioxidized selectively at one sulfur via a one-step or via a two step method (Scheme 10).

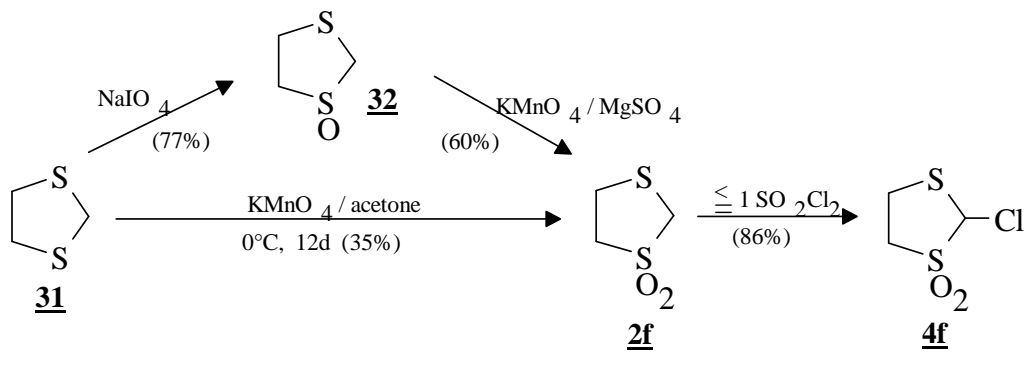

Scheme 10: Synthesis of $\underline{\mathbf{2 f}}$ 
The two 1,3-dithiolane dioxides described in literature ${ }^{29,30}$ were different from $\underline{\mathbf{2 f}}$ which was easily mono-(or bis-) chlorinated at C-2.

In order to determine the relative position of substituents in cyclopropanes $\underline{\mathbf{7}}$ and $\underline{\mathbf{1 6}}$ unambiguously, $\underline{\mathbf{7 a}}, \underline{\mathbf{7 q}}$ and $\underline{\mathbf{1 6 b}}$ were subjected to ${ }^{1} \mathrm{H}-\mathrm{NMR}$ shift measurements with $\mathrm{Eu}(\mathrm{FOD})_{3}$ as earlier described ${ }^{1,30 a}$. In addition, the absolute structure of $\underline{\mathbf{1 6 b}}$ has been independently confirmed via X-ray analysis ${ }^{21 a}$ (Formula 5, Fig. I, Fig. II, Fig. III, Fig. IV).
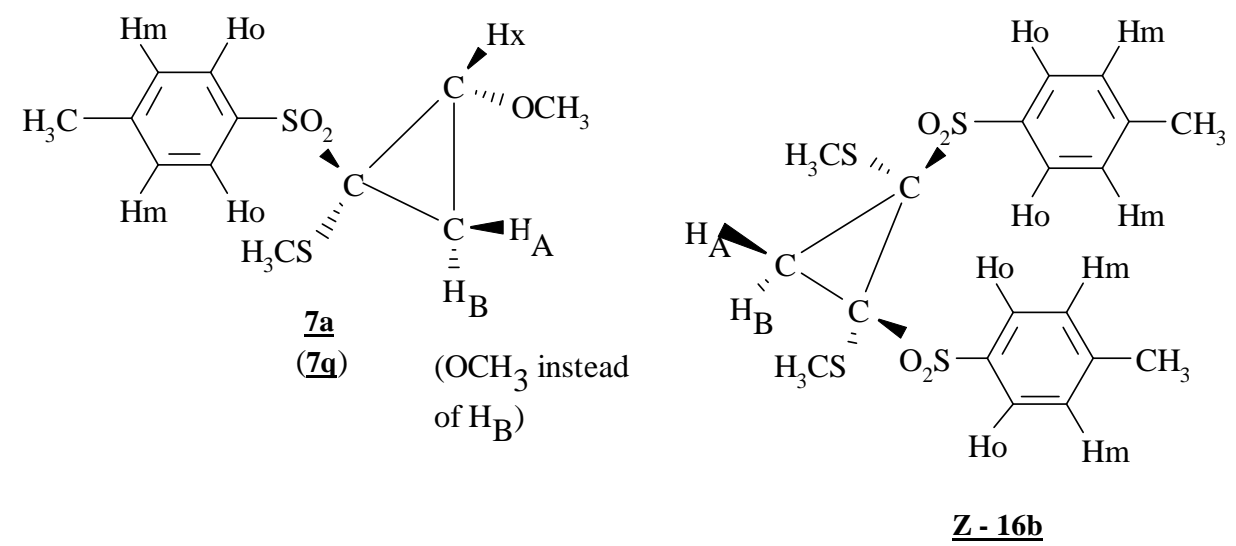

Formula 5: Stereochemistry of trapped cyclopropanes $\underline{\mathbf{7 a}}, \underline{\mathbf{7 g}}$ and $\underline{\mathbf{Z}-\mathbf{1 6} \mathbf{b}}$

In 7a $\mathrm{H}_{\mathrm{X}}$ (7q: $\mathrm{H}_{\text {cyclopropane }}$ ) exhibited the strongest shift, in $\underline{\mathbf{Z}-\mathbf{1 6} \mathbf{b}} \mathrm{H}_{\mathrm{A}}$ exhibited the strongest shift after gradual addition of $\mathrm{Eu}(\mathrm{FOD})_{3}$. All other slopes of the shift diagrams were calculated relative to these basis slopes (Table II).

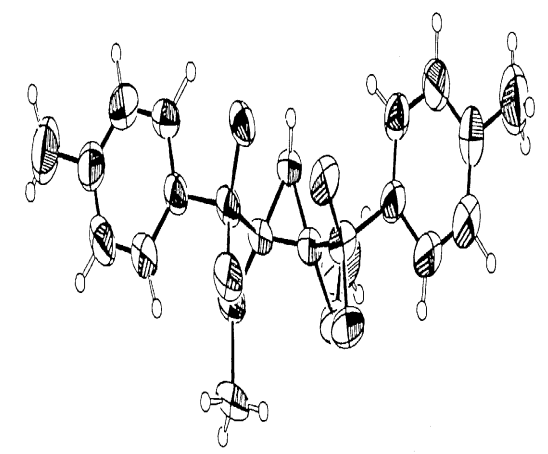

Fig. I: Contour plot of cis-1,2-bis(4-methylphenylsulfonyl)-trans-1,2-dimethylthio-cyclopropane $(\underline{\mathbf{Z}-16 \mathbf{b}})^{*}$

Table II: $\mathrm{Eu}(\mathrm{FOD})_{3}$-shift experiments with $\underline{\mathbf{7 a}, \mathbf{7 q}}$ and $\underline{\mathbf{Z}-\mathbf{1 6} \mathbf{b}}$

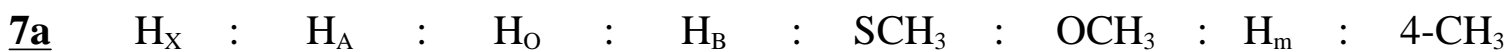
(4.112)
(2.119)
(7.816) (1.469)
(2.186)
(7.346) (7.346)
$(2.447) \quad \delta(\mathrm{ppm})$

$1: 1: 0.82: 0.48: 0.4: 0.23: 0.13: 0.03$

$\underline{7 q}$

\begin{tabular}{|c|c|c|c|c|c|}
\hline $\begin{array}{l}\mathrm{H}_{\text {cycloprop }} \\
(4.11)\end{array}$ & $\begin{array}{l}\mathrm{OCH}_{3} \\
(3.34)\end{array}$ & $\begin{array}{c}\mathrm{H}_{\mathrm{o}} \\
(7.95)\end{array}$ & $\begin{array}{l}\mathrm{SCH}_{3} \\
(2.31)\end{array}$ & $\begin{array}{ll}\mathrm{H}_{\mathrm{m}} \\
(7.46)\end{array}$ & $\begin{array}{r}: \mathrm{ArCH}_{3} \\
(2.48)\end{array}$ \\
\hline 1 & 0.38 & 0.35 & 0.29 & 0.11 & 0.05 \\
\hline
\end{tabular}

$\underline{\text { Z-16b }}$

$$
\begin{aligned}
& \mathrm{H}_{\mathrm{A}}: \mathrm{H}_{\mathrm{B}}: \mathrm{SCH}_{3}: \mathrm{H}_{\mathrm{O}}: \mathrm{H}_{\mathrm{m}}:{ }^{2} \mathrm{CH}_{3} \\
& \begin{array}{llllllll}
(3.274) & (1.901) & (2.135) & (7.979) & (7.348) & (2.461) & \mathrm{d}(\mathrm{ppm})
\end{array} \\
& 1: 0.34: 0.22: 0.19: 2 \times 10^{-4}: \quad 1 \times 10^{-4}
\end{aligned}
$$

On basis of these values structures of all other cyclopropanes $\underline{\mathbf{7}}$ were assigned. 


\section{GENERATION OF CARBENE DIMERS.}

Usually, carbene generation is accompanied by carbene dimerization, however, in the aforementioned carbene reactions no traces of dimerization products could be observed in the presence of strong bases. Therefore, $\alpha$-trimethylsilyl sulfones $\underline{\mathbf{3 3}}$ of higher $\mathrm{CH}$-acidity have been synthesized ${ }^{31}$ which should be chlorinated to the corresponding $\alpha$-chloro $\alpha$-trimethylsilyl sulfones $\underline{\mathbf{3 4}}$ under aprotic conditions (Scheme 11).

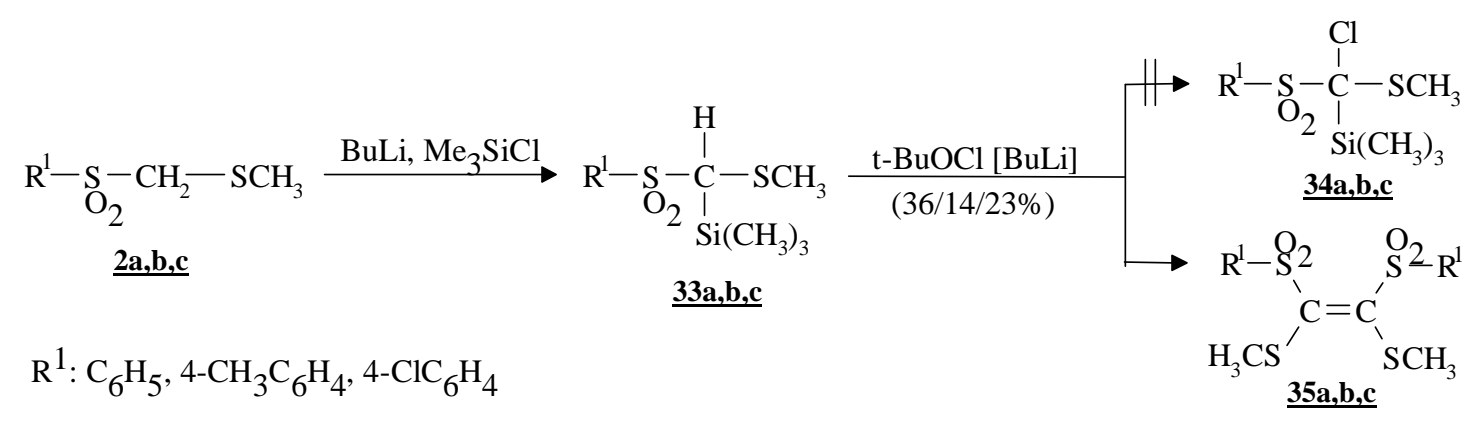

Scheme 11: Ostensible silylative dimerization of title carbenes, method I

No traces of chloro silyl sulfones $\underline{\mathbf{3 4}}$ could be isolated, instead the high melting, well crystallizing dimers $\underline{\mathbf{3 5}}$ were formed $(\mathrm{Z} / \mathrm{E}$ ratios $>95 / 5)$. By-products were $: \underline{\mathbf{2}}, \underline{\mathbf{3 3}}$, monochlorinated $\underline{\mathbf{4}}$ as well as bischlorinated $\underline{\mathbf{1 2}}$ besides traces of $\beta$-disulfones $\underline{\mathbf{2 8}}$.

If the chlorinating agent tert. butylhypochlorite was replaced by hexachloroethane carbene dimer $\mathbf{3 5}$ to B-disulfone $\underline{\mathbf{2 8}} \sim 1: 1$ mixtures were obtained, replacement of hexachloroethane by carbon tetrachloride resulted in a predominating formation of $\beta$-disulfones $\mathbf{2 8}$ besides trace amounts of carbene dimers $\mathbf{3 5}$.

When the above chlorination of $\mathbf{3 3 a}$ with tert. butylhypochlorite was carried out in the presence of dimethoxyethylene ( $\underline{\mathbf{6 h}}: \mathrm{E} / \mathrm{Z}=1 / 3$ mixture, excess), no carbene dimer $\underline{\mathbf{3 5} \mathbf{a}}$ was formed, instead cyclopropane $\underline{\mathbf{7 p}}$ was main product in $36 \%$ yield (cf. table I).

Initial silylation was the preferred reaction of Scheme 11, then chlorination of the anions of $\underline{\mathbf{3 3}}$ was joining. In order to study the significance of the silyl group, Scheme 12 describes inversion of Scheme 11, i.e. $\alpha$-chloro sulfones $\underline{\mathbf{4}}$, their dichlorinated analogs $\underline{\mathbf{1 2}}$ or their brominated products $\underline{\mathbf{1 3}}$ served as carbanion precursors. As shown in Scheme 11 carbanionization with BuLi in the presence of trimethylsilyl chloride led to immediate formation of carbene dimers $\underline{\mathbf{3 5}}$ in somewhat lower yields (Scheme 12). Correspondingly, tetraarylthiosubstituted dimer $\underline{\mathbf{3 5} \mathbf{e}}$ was accessible in low yields ( $2 \%$ from $\underline{\mathbf{4 e}}$ respectively $11 \%$ from $\underline{\mathbf{1 2} \mathbf{e})}$.
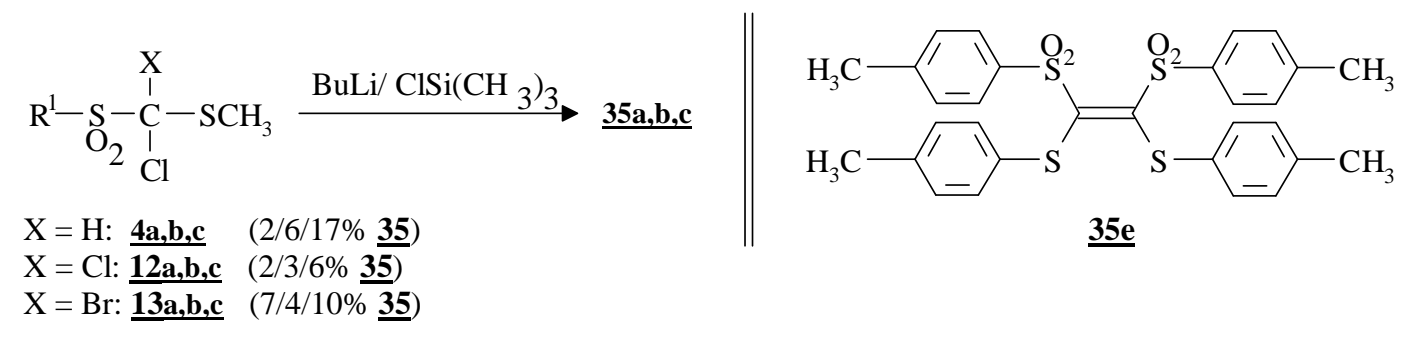

Scheme 12: Ostensible silylative dimerization of the title carbenes, method II

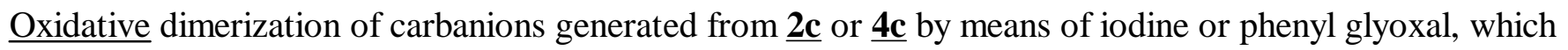
usually prefer one-electron-oxidations, also gave $\mathbf{3 5 c}$ but in poor yields (Scheme 13).

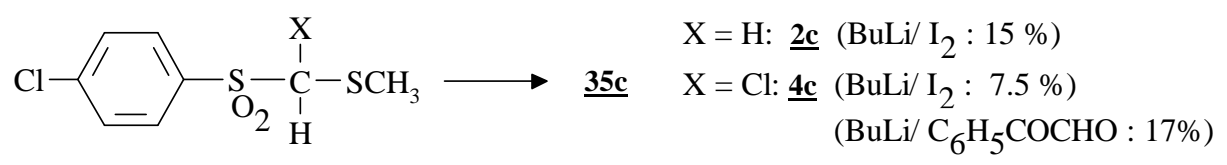

Scheme 13: Oxidative C,C-connections yielding $\underline{\mathbf{3 5}}$, method III

Attempted reductive C,C-connections starting from dichloro sulfonyl thioether 12c, from bromochloro sulfonyl thioether $\underline{\mathbf{1 3 c}}$ or from its dibromo analogue (not described here in detail) by means of copper, zinc, or 
magnesium were unsuccessful. Neither formation of dimer $\underline{\mathbf{3 5 c}}$ nor of carbene trapping product $\underline{\mathbf{7 r}}$ with 1,2-dimethoxyethylene were observed.

Methods I-III yielded nearly pure $\underline{\mathbf{Z}}$-isomers $\underline{\mathbf{3 5}}$ as was observed in other cases (cf. the cis-effect ${ }^{32,33}$ ). Isomerizations $>90 \%$ yielding the corresponding E-isomers $\mathbf{3 5}$ resulted from addition of molar amounts of bromine in chloroform solution; bromine could be replaced neither by iodine (no reaction) nor by chlorine (decomposition).

E-35 isomers proved to be rather unstable under normal conditions, they reisomerized as in the solid state as in solution to form their Z-isomers $\mathbf{3 5}$ (Scheme 14).

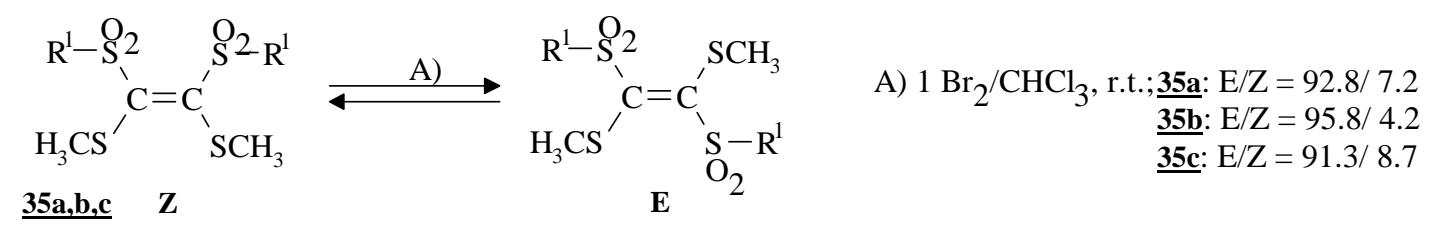

Scheme 14: Reversible isomerizations of carbene dimers $\underline{\mathbf{3 5}}$

As outlined in table I (cyclopropanes $\underline{\mathbf{7 v}}, \underline{\mathbf{w}}, \underline{\mathbf{x}}$ ) cyclic carbenes $\underline{\mathbf{3 f}}, \mathbf{g}, \underline{\mathbf{h}}$ could be generated from the corresponding $\alpha$-chloro $\alpha$-sulfonyl thioethers $\underline{\mathbf{4 f}}, \mathbf{g}, \underline{\mathbf{h}}$ as described for open chain analogs. However, their formal dimers $\underline{\mathbf{3 5 f}}, \mathbf{g}, \underline{\mathbf{h}}$ were not accessible as described before; likewise, attempts starting from the corresponding trimethylsilyl sulfonyl thioethers $\underline{\mathbf{3 3}}, \mathbf{g}, \underline{\mathbf{h}}(\underline{\mathbf{3 3 f}}$ not accessible via described preparation procedure in consequence of carbanion instablility of $\underline{\mathbf{2 f}}$ ) failed (Scheme 15).

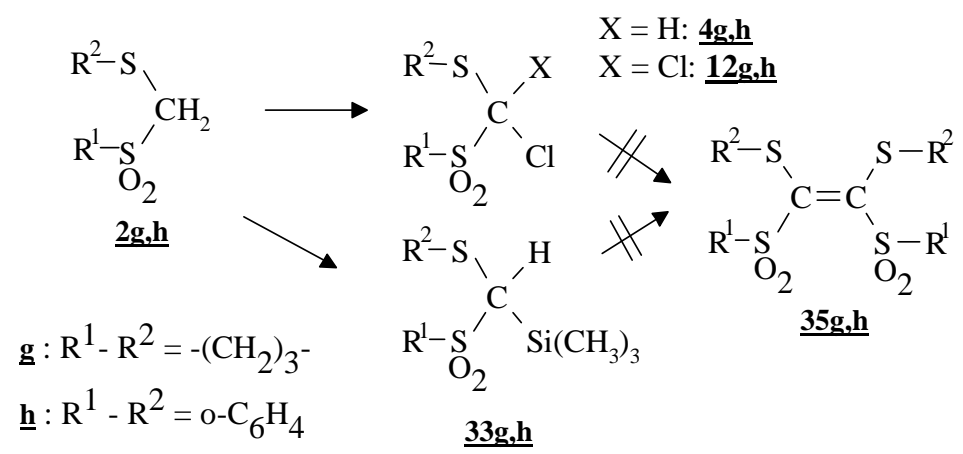

Scheme 15: Unsuccessful attempts to synthesize cyclic carbene dimers $\underline{\mathbf{3 5 g}}, \underline{\mathbf{h}}$

In the case of $\underline{\mathbf{3 5 h}}$ this failure was surprising since corresponding open chain tetraaryl sulfur substituted ethylene $\underline{35 \mathrm{e}}\left(\mathrm{R}^{1}, \mathrm{R}^{2}=4-\mathrm{CH}_{3} \mathrm{C}_{6} \mathrm{H}_{4}\right)$ could be obtained as in the above mentioned cases.

\section{CONCLUSION}

Organothio sulfonyl carbenes $\underline{\mathbf{3}}$ proved to be electrophilic carbenes similar to their methoxy analogs ${ }^{1}$. Prepared in situ via ylid decomposition (method A) or via $\alpha$-eliminations (method B) they could be trapped by sufficiently nucleophilic reagents (i.e. sulfinate anions, diazo alkanes, enolethers, styrenes).

The usual carbene dimerizations were prevented by traces of sulfinate anions. After silylative removal of sulfinate impurifications ${ }^{34}$ trapping of the Z-dimers was observed for the first time. Z/E isomerizations proved to be easily reversible.

A series of sulfur substituted cyclopropanes as well as several types of heterocyclic compounds were easily accessible via the title carbenes. Structure elucidations were afforded by ${ }^{1} \mathrm{H}-\mathrm{NMR}$ shift measurements and X-ray analysis. 


\section{EXPERIMENTAL PART}

Melting points were determined using a Kofler apparatus and/or a Fus-O-mat ${ }^{35}$ of Heraeus. Elemental analyses ${ }^{36}$ were in agreement with the calculated values. Infrared spectra were recorded on Beckman IR4230 or IR33 spectrometers. ${ }^{1} \mathrm{H}$ and ${ }^{13} \mathrm{C}$ NMR spectra were recorded predominantly on Bruker WH90 and AM400 spectrometers of $\mathrm{CDCl}_{3}$ solutions (TMS as internal standard). TLC used Alugram $^{\oplus}$ SIL G/UV ${ }_{254}$ foils from Macherey and Nagel. A 1-2:3 E/Z mixture of 1,2-dimethoxyethylene isomers ${ }^{1}$ was separated by use of a micro spinning band column from NORMAG (100 cm length, $1200 \mathrm{cpm})$, fractions were monitored by GC using a Hewlett-Packard 5750 G.

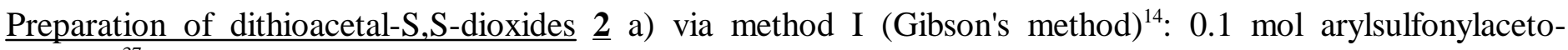
phenone $^{37}, 10.6 \mathrm{~g},(0.1 \mathrm{~mol})$ sodium carbonate, and $0.1 \mathrm{~mol}$ methylthio methanesulfonate or methylthio 4-chlorobenzenesulfonate were heated with stirring in $200 \mathrm{ml}$ ethanol until the evolution of carbon dioxide stopped (1-2 h). The reaction mixture was added to $500 \mathrm{ml}$ ice water and the immediately formed arylsulfonyl thioethers $\underline{\mathbf{2}}$ were extracted with dichloromethane; occasionally, acyl cleavages did not proceed completely. In these cases the crystalline and insoluble precursors were cleaved by an additional heating with dilute aqueous-alcoholic sodium hydroxide solution. In the case of $\mathbf{2 c}$ thiolation must be carried out by means of methylthio 4-chlorobenzenesulfonate, otherwise the 4-chlorobenzenesulfonyl group was easily substituted as the corresponding sulfinate by more nucleophilic methanesulfinate formed during conversion. In accordance with literature data, the following arylsulfonyl methylthiomethylsulfones were obtained: $\underline{\mathbf{2 a}}\left(\mathrm{R}^{1}=\mathrm{C}_{6} \mathrm{H}_{5}\right)$, 80\%, m.p. $84^{\circ} \mathrm{C}$ (methanol) ${ }^{14 \mathrm{~b}} ; \underline{\mathbf{2 b}}\left(\mathrm{R}^{1}=4-\mathrm{CH}_{3}-\mathrm{C}_{6} \mathrm{H}_{4}\right), 85 \%$, m.p. $80^{\circ} \mathrm{C}$ (methanol) ${ }^{14 \mathrm{a}} ; \underline{\mathbf{2 c}}\left(\mathrm{R}^{1}=4-\mathrm{Cl}-\mathrm{C}_{6} \mathrm{H}_{4}\right), 85 \%$, m.p. $92^{\circ} \mathrm{C}$ (methanol) $)^{14 \mathrm{~b}}$.

b) via method II (Selective unsymmetrical bisoxidation of 1,3-dithiolane $\left.(\underline{\mathbf{3 1}})^{28}\right)$; One-step method: A solution of $4.25 \mathrm{~g}$ ( $40 \mathrm{mmol}$ ) 1,3-dithiolane in $200 \mathrm{ml}$ acetone was stirred at $0^{\circ} \mathrm{C}$ for $12 \mathrm{~d}$ during gradual addition of 12.64 $\mathrm{g}(80 \mathrm{mmol})$ of potassium permanganate. The inorganic precipitate was removed by filtration, the solvent was distilled off, finally i. vac., and a few $\mathrm{ml}$ of absolute ether were added. The resulting colorless crystals of 1,3-dithiolane-1,1-dioxide $(\underline{\mathbf{2 f}})$ were recrystallized from ethanol, $1.93 \mathrm{~g}(35 \%)$, m.p. $73.5^{\circ} \mathrm{C}$. This substance was not identical with the two 1,3-dithiolane dioxides mentioned already in the literature (m.p. $134{ }^{\circ} \mathrm{C}^{29}$; m.p. 157-158.5 $\left.{ }^{\circ} \mathrm{C}^{30}\right) . \mathrm{C}_{3} \mathrm{H}_{6} \mathrm{O}_{2} \mathrm{~S}_{2}$ (138.21); $\mathrm{M}^{+}=138$; calc. C $26.07 \mathrm{H}$ 4.38. found C $26.1 \mathrm{H} 4.29$; IR (KBr): 1305, 1125, $1110\left(\mathrm{SO}_{2}\right) \mathrm{cm}^{-1}$; ${ }^{1} \mathrm{H}-\mathrm{NMR}\left(\mathrm{CDCl}_{3} / \mathrm{TMS}\right): \delta=3.203-3.237\left(\mathrm{~m}, 2 \mathrm{H}, \mathrm{SCH}_{2}\right), 3.312-3.346(\mathrm{~m}, 2 \mathrm{H}$, $\mathrm{SO}_{2} \mathrm{CH}_{2}$ ), 3.865 (s, 2H, $\left.\mathrm{SCH}_{2} \mathrm{SO}_{2}\right)$ ppm; ${ }^{13} \mathrm{C}-\mathrm{NMR}\left(\mathrm{CDCl}_{3} / \mathrm{TMS}\right): \delta=52.04(\mathrm{C}-2), 25.27(\mathrm{C}-4), 48.37(\mathrm{C}-5)$ ppm.

Two-step-method: A molar amount of sodium periodate in water $(0.1 \mathrm{~mol}$ in $300 \mathrm{ml})$ was added dropwise under stirring at $0{ }^{\circ} \mathrm{C}$ to solutions of the cyclic dithioacetals 1,3 -dithiolane ${ }^{28}, 1,3$-dithiane ${ }^{39}$ or 1,3 -benzodithiol ${ }^{40}$ in methanol $(0.1 \mathrm{~mol}$ in $1000 \mathrm{ml})$. Stirring was continued overnight. Precipitated sodium iodate was removed by filtration and the solvent was evaporated i. vac. at room temperature. The residue was dissolved in chloroform, dried with magnesium sulfate, and the solvent was removed for a second time. 1,3-Dithiolane-1-oxide resulted as an oil (77\% yield), 1,3-dithiane-S-oxide crystallized after addition of a few ml of dry ether, m.p. $89{ }^{\circ} \mathrm{C}^{41}(95 \%$ yield), 1,3-benzodithiol-1-oxide (94\% yield) afforded colorless crystals after recrystallization from methanol, m.p. 93-94 ${ }^{\circ} \mathrm{C}$; structural confirmations of these sulfoxides were carried out via further oxidation to their corresponding sulfones: A solution of $10.6 \mathrm{~g}(0.067 \mathrm{~mol})$ potassium permanganate in $470 \mathrm{ml}$ water was dropped under stirring at room temperature to a combined solution of the corresponding dithioacetaloxide $(0.067 \mathrm{~mol}$ in 11 dioxane) and of $33.6 \mathrm{~g}(0.28 \mathrm{~mol})$ magnesium sulfate in 11 water. Stirring was continued for $12 \mathrm{~h}$ and the solvent was removed i. vac. after filtration. The residue was extracted three times with $200 \mathrm{ml}$ dichloromethane, the combined extracts were dried over magnesium sulfate and the solvent was removed. The sulfones $\underline{\mathbf{2 f}}, \underline{\mathbf{2 g}} \underline{\mathbf{2 h}}$ crystallized after addition of a few $\mathrm{ml}$ of dry ether, $\mathbf{2 f}$ (60\% yield) and $\mathbf{2} \mathbf{g}$ ( $100 \%$ yield) were recrystallized from ethanol, $\underline{\mathbf{2 h}}$ (73\% yield) was recrystallized from methanol. The m.p. $73.5^{\circ} \mathrm{C}$ of $\underline{\mathbf{2 f}}$ was identical with that of the one-pot procedure, the m.p. $138.5^{\circ} \mathrm{C}$ of $\mathbf{2 g}$ corresponded to the literature ${ }^{42}, 1,3$-benzodithiol-1,1-dioxide (2h): m.p. $55^{\circ} \mathrm{C} ; \mathrm{C}_{7} \mathrm{H}_{6} \mathrm{O}_{2} \mathrm{~S}_{2}$ (186.3); $\mathrm{M}^{+}=186$; calc. $\mathrm{C} 45.14 \mathrm{H} \mathrm{3.25}$, found $\mathrm{C} 45.1 \mathrm{H} 3.24$; IR (KBr): 1320, 1185, $1150\left(\mathrm{SO}_{2}\right) \mathrm{cm}^{-1} ;{ }^{1} \mathrm{H}-\mathrm{NMR}\left(\mathrm{CDCl}_{3} / \mathrm{TMS}\right): \delta=4.368\left(\mathrm{~s}, 2 \mathrm{H}, \mathrm{CH}_{2}\right), 7.348-7.386\left(\mathrm{~m}, 2 \mathrm{H}_{\mathrm{ar}}\right), 7.536-7.575(\mathrm{~m}, 1$ $\left.\mathrm{H}_{\mathrm{ar}}\right), 7.707-7.730\left(\mathrm{~m}, 1 \mathrm{H}_{\mathrm{ar}}\right) \mathrm{ppm} ;{ }^{13} \mathrm{C}\left(\mathrm{CDCl}_{3} / \mathrm{TMS}\right): \delta=49.78\left(\mathrm{CH}_{2}\right), 123.17,125.57,126.59,133.98,134.06$, $134.24\left(6 \mathrm{C}_{\mathrm{ar}}\right) \mathrm{ppm}$.

c) via method IIIa ("Ogura's method") ${ }^{16}$ : According to the described procedure starting from DMSO and acetic anhydride, in situ formed methylthiomethyl acetate was converted with sodium arenesulfinates $\underline{\mathbf{2 3 a}}, \underline{\mathbf{2 3 b}}$ to afford $\underline{\mathbf{2 a}}, \underline{\mathbf{2 b}}$ in larger amounts and in a high purity. 
d) via method IIIb: Aryl chloromethyl sulfones were easily accessible by a procedure described by Bordwell and Cooper $^{43}$. Further conversions with excess sodium methylthiolate in ethanol have been described frequently ${ }^{44 a-c}$ sulfones $\underline{\mathbf{2} \mathbf{a}}(87 \%), \underline{\mathbf{2 b}}(85 \%), \underline{\mathbf{2 c}}(81 \%)$ could be obtained by this method in high yields. Conversions of aryl bromomethyl sulfones ${ }^{45)}$ with arenethiolates gave lower yields.

e) via method IV (sulfinate-sulfone Pummerer rearrangement $\left.{ }^{18}\right)$ : $10 \mathrm{~g}(0.1 \mathrm{~mol})$ acetylacetone $\underline{\mathbf{8}}\left(\mathrm{R}^{3}, \mathrm{R}^{4}=\mathrm{CH}_{3}\right)$ and $15.8 \mathrm{~g}(0.2 \mathrm{~mol})$ of dry pyridine were dissolved in $200 \mathrm{ml}$ of absol. THF. After stirring and cooling with an ice salt mixture, $34.8 \mathrm{~g}(0.2 \mathrm{~mol})$ pure p-toluenesulfinylchloride $(\mathbf{9 b})$ (purified by short path distillation i. Vac. ${ }^{46}$ ) in $30 \mathrm{ml}$ absol. THF were added dropwise below $-5^{\circ} \mathrm{C}$ and stirring was continued for $20 \mathrm{~h}$ at room temperature. Precipitated pyridine hydrochloride was removed by filtration, and the solvent was removed i. vac. by a rotary evaporator. $11\left(\mathrm{R}^{3}, \mathrm{R}^{4}=\mathrm{CH}_{3}, \mathrm{R}^{1}, \mathrm{R}^{2}=4-\mathrm{CH}_{3} \mathrm{C}_{6} \mathrm{H}_{4}\right)$ was obtained in a crude yield of $92 \%$. After recrystallization from methanol $29.3 \mathrm{~g}$ (78\%), m.p. $125{ }^{\circ} \mathrm{C}$, were obtained; $\mathrm{C}_{19} \mathrm{H}_{20} \mathrm{O}_{4} \mathrm{~S}_{2}$ (376.5), calc $\mathrm{C} 60.61 \mathrm{H} 5.35$ found $\mathrm{C}$ $60.83 \mathrm{H}$ 5.38; IR (KBr): 1710, $1695(\mathrm{C}=\mathrm{O}), 1315,1180,1140\left(\mathrm{SO}_{2}\right) \mathrm{cm}^{-1},{ }^{1} \mathrm{H}-\mathrm{NMR}\left(\mathrm{CDCl}_{3} / \mathrm{TMS}\right): \delta=2.32(6$ $\mathrm{H}, \mathrm{CH}_{3} \mathrm{CO}$ and $\left.4-\mathrm{CH}_{3} \mathrm{C}_{6} \mathrm{H}_{4} \mathrm{~S}\right), 2.44\left(3 \mathrm{H}, 4-\mathrm{CH}_{3} \mathrm{C}_{6} \mathrm{H}_{4} \mathrm{SO}_{2}\right), 7.0-8.1\left(\mathrm{~m}, 8 \mathrm{H}_{\mathrm{ar}}\right) \mathrm{ppm}$.

$25.3 \mathrm{~g}(67 \mathrm{mmol})$ sulfone 11 were refluxed with a mixture of $10 \mathrm{~g}(175 \mathrm{mmol})$ potassium hydroxide in $200 \mathrm{ml}$ ethanol and $40 \mathrm{ml}$ water for $90 \mathrm{~min}$. The solvent was removed i. vac. and the residue was recrystallized from methanol, m.p. $76{ }^{\circ} \mathrm{C}$ according to literature ${ }^{47)}$; yield $15 \mathrm{~g}(77 \%)$.

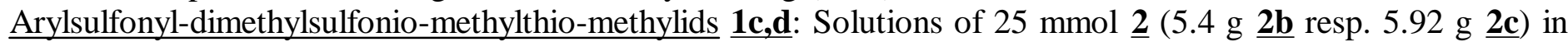
$60 \mathrm{ml}$ absol. THF were metalated at $-78^{\circ} \mathrm{C}$ under nitrogen by slow addition of $53 \mathrm{mmol}$-BuLi in hexane $(21 \mathrm{ml}$ of a 2.5-molar solution) and stirring for $90 \mathrm{~min}$. A suspension of $\underline{\mathbf{5}} \mathrm{in} 25 \mathrm{ml}$ absol. THF (prepared from $3.94 \mathrm{~g}$ (29.4 mmol) N-chlorosuccinimide and $2 \mathrm{~g}(29.4 \mathrm{mmol})$ dimethyl sulfide in $75 \mathrm{ml}$ absol. dichloromethane) was slowly added and stirring was continued overnight at room temperature. The precipitated ylids $\underline{\mathbf{1 c}, \mathbf{d}}$ were separated by filtration: Dimethyl sulfonio-4-methylphenylsulfonyl-methylthio-methylid (1c), $0.7 \mathrm{~g}$ (10\%), colorless crystals, m.p. $143{ }^{\circ} \mathrm{C} ; \mathrm{C}_{11} \mathrm{H}_{16} \mathrm{O}_{2} \mathrm{~S}_{3}$ (276.4), calc. C $47.79 \mathrm{H} 5.83$ found $\mathrm{C} 47.68 \mathrm{H} \mathrm{5.78;} \mathrm{IR} \mathrm{(KBr):} \mathrm{1255,}$ $1130\left(\mathrm{SO}_{2}\right) \mathrm{cm}^{-1} ;{ }^{1} \mathrm{H}-\mathrm{NMR}\left(\mathrm{CDCl}_{3} / \mathrm{TMS}\right): \delta=2.152\left(\mathrm{~s}, 3 \mathrm{H}, \mathrm{SCH}_{3}\right), 2.373\left(\mathrm{~s}, 3 \mathrm{H}, \mathrm{ArCH}_{3}\right), 2.561(\mathrm{~s}, 6 \mathrm{H}$, $\left.\mathrm{S}^{+}\left(\mathrm{CH}_{3}\right)_{2}\right), 7.208\left(\mathrm{~d}, \mathrm{~J}=8 \mathrm{~Hz}, 2 \mathrm{H}_{\mathrm{ar}}\right), 7.747\left(\mathrm{~d}, \mathrm{~J}=8.2 \mathrm{~Hz}, 2 \mathrm{H}_{\mathrm{ar}}\right)$ ppm. 4-Chlorophenylsulfonyldimethylsulfonio-methylthio-methylid (1d $), 1.4 \mathrm{~g}$ (19\%), colorless crystals, m.p. $130{ }^{\circ} \mathrm{C} ; \mathrm{C}_{10} \mathrm{H}_{13} \mathrm{ClO}_{2} \mathrm{~S}_{3}(296,9)$, calc. C $40.46 \mathrm{H} 4.41$ found $\mathrm{C} 40.56 \mathrm{H} 4.40$; IR $(\mathrm{KBr}): 1270,1260,1150,1140\left(\mathrm{SO}_{2}\right) \mathrm{cm}^{-1}$; ${ }^{1} \mathrm{H}-\mathrm{NMR}$ $\left(\mathrm{CDCl}_{3} / \mathrm{TMS}\right): \delta=2.179\left(\mathrm{~s}, 3 \mathrm{H}, \mathrm{SCH}_{3}\right), 2.562\left(\mathrm{~s}, 6 \mathrm{H}, \mathrm{S}^{+}\left(\mathrm{CH}_{3}\right)_{2}\right), 7.383\left(\mathrm{~d}, \mathrm{~J}=8.2 \mathrm{~Hz}, 2 \mathrm{H}_{\mathrm{ar}}\right), 7.804(\mathrm{~d}, \mathrm{~J}=8.3$ $\left.\mathrm{Hz}, 2 \mathrm{H}_{\mathrm{ar}}\right) \mathrm{ppm} ;{ }^{13} \mathrm{C}-\mathrm{NMR}\left(\mathrm{CDCl}_{3} / \mathrm{TMS}\right): \delta=25.02\left(\mathrm{SCH}_{3}\right), 32.27\left(\mathrm{~S}^{+}\left(\mathrm{CH}_{3}\right)_{2}\right), 51.66$ (ylid-C), 127.32, 128.70, $136.82,145.20\left(\mathrm{C}_{\mathrm{ar}}\right) \mathrm{ppm}$.

Thermal decomposition of 1d and trapping of carbene intermediate $\mathbf{3 d}$ in boiling Z,E-dimethoxyethylene (6f): $250 \mathrm{mg}(0.84 \mathrm{mmol}) \underline{1 c}$ and $5.28 \mathrm{~g}(60 \mathrm{mmol}) \underline{6 f}$ were refluxed for $2 \mathrm{~d}$. Aqueous work-up, extraction with ether, and usual removal of volatile components afforded a crude residue which exhibited the characteristic ${ }^{1} \mathrm{H}-\mathrm{NMR}$ signals of cyclopropane $\mathbf{7 r}$ (isolation and characterization of $\mathbf{7 r}$ see later).

Preparation of halogenated sulfonyl thioethers $\underline{\mathbf{4}}, \underline{\mathbf{1 2}}, \underline{\mathbf{1 3}}$. Arylsulfonylmonochloromethyl thioethers $\underline{\text { 4a-h }}$ (except d), general procedure: $5.4 \mathrm{~g}$ (40 mmol) sulfuryl chloride in $50 \mathrm{ml}$ absol. dichloromethane were dropped very slowly to a boiling solution of dithioacetal-S,S-dioxide $\mathbf{4 a - g}$ (except $\underline{\mathbf{d}}$ ) (40-44 mmol) in $50 \mathrm{ml}$ absol. dichloromethane. Boiling was continued for $1 \mathrm{~h}$ after addition, then the solvent was removed i. vac. and the residue was crystallized from methanol. Only in the case of $\underline{\mathbf{4 h}}$ Ogura's method ${ }^{21 \mathrm{c}}$ in chloroform at $0{ }^{\circ} \mathrm{C}$ had been applied, using a ratio of $21 \mathrm{mmol} \underline{\mathbf{4 h}}$ to $50 \mathrm{mmol}(4 \mathrm{ml})$ sulfuryl chloride; no dichlorination occurs in this case.

[Chloro-(methylthio)-methyl] phenyl sulfone (4a): $78 \%$ colorless oil, b.p. $134{ }^{\circ} \mathrm{C} / 0.02$ Torr; $\mathrm{C}_{14} \mathrm{H}_{12} \mathrm{Cl}_{2} \mathrm{O}_{2} \mathrm{~S}_{2}$ (347.3), calc. C $48.42 \mathrm{H} 3.38$ found C $48.26 \mathrm{H} 3.40$; IR (neat) 1340, 1315, 1170, $1155\left(\mathrm{SO}_{2}\right) \mathrm{cm}^{-1}$; ${ }^{1} \mathrm{H}-\mathrm{NMR}$ $\left(\mathrm{CDCl}_{3} / \mathrm{TMS}\right): \delta=2.451\left(\mathrm{~s}, 3 \mathrm{H}, \mathrm{SCH}_{3}\right), 5.578(\mathrm{~S}, 1 \mathrm{H}, \mathrm{CH}), 7.599\left(\mathrm{t}, \mathrm{J}=6.7 \mathrm{~Hz}, 2 \mathrm{H}_{\mathrm{ar}}\right), 7.664-7.775(\mathrm{~m}, 1$ $\left.\mathrm{H}_{\mathrm{ar}}\right), 7.985\left(\mathrm{~d}, \mathrm{~J}=7.5 \mathrm{~Hz}, 2 \mathrm{H}_{\mathrm{ar}}\right) \mathrm{ppm} ;{ }^{13} \mathrm{C}-\mathrm{NMR}\left(\mathrm{CDCl}_{3} / \mathrm{TMS}\right): \delta=13.39\left(\mathrm{SCH}_{3}\right), 77.69(\mathrm{CH}), 128.81,129.06$, 129.97, 132.29, 133.93, $134.63\left(\mathrm{C}_{\mathrm{ar}}\right) \mathrm{ppm}$. [Chloro-(methylthio)-methyl] 4-methylphenyl sulfone (4b): 94\%, colorless crystals, m.p. $102.5{ }^{\circ} \mathrm{C}$ (ref. 21c: $100.5-101{ }^{\circ} \mathrm{C}$, 73\%). [Chloro-(methylthio)-methyl] 4-chlorophenyl sulfone (4c): 82\%, colorless crystals, m.p. $70-71{ }^{\circ} \mathrm{C} ; \mathrm{C}_{8} \mathrm{H}_{8} \mathrm{Cl}_{2} \mathrm{O}_{2} \mathrm{~S}_{2}$ (271.2) calc. C 35.43, $\mathrm{H} 2.97$ found C 34.94 $\mathrm{H}$ 2.90; IR (KBr): 1330, 1153, $1145\left(\mathrm{SO}_{2}\right) \mathrm{cm}^{-1} ;{ }^{1} \mathrm{H}-\mathrm{NMR}\left(\mathrm{CDCl}_{3} / \mathrm{TMS}\right)^{48}: \delta=2.50\left(\mathrm{~s}, 3 \mathrm{H}, \mathrm{SCH}_{3}\right), 5.60(\mathrm{~s}, 1 \mathrm{H}$,

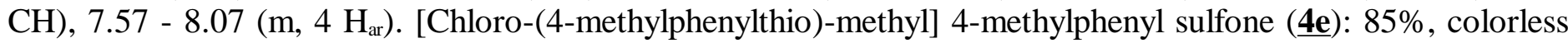
crystals, m.p. $101{ }^{\circ} \mathrm{C}$, (ref. $21 \mathrm{~d}$ : $105-107{ }^{\circ} \mathrm{C}, 83 \%$; This literature method yields absolutely pure substances whereas the chlorination method affords products which contain small amounts of unreacted starting materials $\underline{\mathbf{2}}$ or of dichlorination products $\underline{\mathbf{1 2}}$ or of both $\underline{\mathbf{2}}$ and $\underline{\mathbf{1 2}}$; thus, m.p. given here represent lower border values). 2-Chloro-1.3-dithiolane-1,1-dioxide (4f): 86\%, colorless crystals, m.p. 71-72.5 ${ }^{\circ} \mathrm{C} ; \mathrm{C}_{3} \mathrm{H}_{5} \mathrm{ClO}_{2} \mathrm{~S}_{2}(172.7)$ calc. C $20.87 \mathrm{H} 2.92$ found C $20.9 \mathrm{H} 2.84$; IR $(\mathrm{KBr}): 1330,1320,1160,1150\left(\mathrm{SO}_{2}\right) \mathrm{cm}^{-1} ;{ }^{1} \mathrm{H}-\mathrm{NMR}\left(\mathrm{CDCl}_{3} / \mathrm{TMS}\right): \delta=$ $3.225-3.302$ (m, $1 \mathrm{H}$ of a $\mathrm{CH}_{2}$-group), $3.399-3.547$ (m, $3 \mathrm{H}, 1 \mathrm{H}$ of the aforementioned $\mathrm{CH}_{2}$ and $2 \mathrm{H}$ of the 
second $\mathrm{CH}_{2}$ ), 5.838 (s, $\left.1 \mathrm{H}, \mathrm{CH}\right)$ ppm. 2-Chloro-1,3-dithiane-1,1-dioxide (4g): 86\%, colorless crystals, m.p. 152 ${ }^{\circ} \mathrm{C} ; \mathrm{C}_{4} \mathrm{H}_{7} \mathrm{ClO}_{2} \mathrm{~S}_{2}$ (186.7) calc. C $25.74 \mathrm{H} 3.78$ found C $26.0 \mathrm{H} \mathrm{3.72;} \mathrm{IR} \mathrm{(KBr):} \mathrm{1320,} \mathrm{1305,} 1175\left(\mathrm{SO}_{2}\right) \mathrm{cm}^{-1}$; ${ }^{1} \mathrm{H}-\mathrm{NMR}\left(\mathrm{CDCl}_{3} / \mathrm{TMS}\right): \delta=2.509-2.618\left(\mathrm{~m}, 2 \mathrm{H}, \mathrm{CH}_{2}\right), 2.699-2.768\left(\mathrm{~m}, 1 \mathrm{H}\right.$ of a $\left.\mathrm{CH}_{2}\right), 3.042-3.087(\mathrm{~m}, 1$ $\mathrm{H}$ of a $\left.\mathrm{CH}_{2}\right), 3.192-3.264\left(\mathrm{~m}, 1 \mathrm{H}\right.$ of a $\left.\mathrm{CH}_{2}\right), 3.637-3.715\left(\mathrm{~m}, 1 \mathrm{H}\right.$ of a $\left.\mathrm{CH}_{2}\right), 5.593(\mathrm{~d}, \mathrm{~J}=2.4 \mathrm{~Hz}, 1 \mathrm{H}, \mathrm{CH})$. 2-Chloro-1,3-benzodithiol-1,1-dioxide (4h): $95 \%$, colorless crystals, m.p. $111-112{ }^{\circ} \mathrm{C} ; \mathrm{C}_{7} \mathrm{H}_{5} \mathrm{ClO}_{2} \mathrm{~S}_{2}(219.8)$, no elemental analysis available; IR (KBr): $1325,1175,1165\left(\mathrm{SO}_{2}\right) \mathrm{cm}^{-1} ;{ }^{1} \mathrm{H}-\mathrm{NMR}\left(\mathrm{CDCl}_{3} / \mathrm{TMS}\right): \delta=6.057(\mathrm{~s}, 1 \mathrm{H}$, $\mathrm{CH}), 7.403\left(\mathrm{~d}, \mathrm{~J}=8.0 \mathrm{~Hz}, 1 \mathrm{H}_{\mathrm{ar}}\right), 7.449-7.485\left(\mathrm{~m}, 1 \mathrm{H}_{\mathrm{ar}}\right), 7.628-7.669\left(\mathrm{~m}, 1 \mathrm{H}_{\mathrm{ar}}\right) ; 7.805-7.827\left(\mathrm{~m}, 1 \mathrm{H}_{\mathrm{ar}}\right)$ ppm; ${ }^{13} \mathrm{C}-\mathrm{NMR}\left(\mathrm{CDCl}_{3} / \mathrm{TMS}\right): \delta=73.62(\mathrm{CH}), 124.87,126.28,127.81,131.13,134.95\left(\mathrm{C}_{\mathrm{ar}}\right) \mathrm{ppm}$.

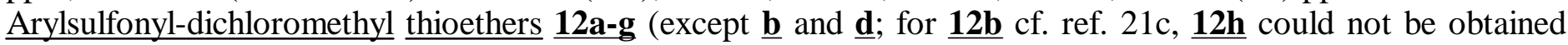
even by use of a larger excess of sulfuryl chloride); general procedure: $225 \mathrm{mmol}$ sulfuryl chloride were dropped slowly to a solution of $100 \mathrm{mmol}$ dithioacetal- S,S-dioxide $\underline{4}$ in $300 \mathrm{ml}$ chloroform with stirring at $0{ }^{\circ} \mathrm{C}$. Stirring was continued for $6 \mathrm{~h}$ at $0{ }^{\circ} \mathrm{C}$, then $12 \mathrm{~h}$ at room temperature. The chloroform solution was washed with $400 \mathrm{ml}$ water, 11 ether was added, and the combined organic phases were washed with 11 of water, then dried over magnesium sulfate. The solvent was evaporated and the residue was recrystallized from methanol. [Dichloro-(methylthio)-methyl] phenyl sulfone (12a): 86\%, colorless crystals, m.p. $51{ }^{\circ} \mathrm{C} ; \mathrm{C}_{8} \mathrm{H}_{8} \mathrm{Cl}_{2} \mathrm{O}_{2} \mathrm{~S}_{2}(271.2)$ calc. C $35.43 \mathrm{H} 2.97$ found C $35.5 \mathrm{H}$ 2.95; IR $(\mathrm{KBr}): 1335,1160\left(\mathrm{SO}_{2}\right) \mathrm{cm}^{-1} ;{ }^{1} \mathrm{H}-\mathrm{NMR}\left(\mathrm{CDCl}_{3} / \mathrm{TMS}\right): \delta=2.647$ $\left(\mathrm{s}, 3 \mathrm{H}, \mathrm{SCH}_{3}\right), 7.605\left(\mathrm{t}, \mathrm{J}=7.9 \mathrm{~Hz}, 2 \mathrm{H}_{\mathrm{ar}}\right), 7.759$ (t, J = 7.5Hz, $\left.1 \mathrm{H}_{\mathrm{ar}}\right),\left(8.093\left(\mathrm{~d}, \mathrm{~J}=7.9 \mathrm{~Hz}, 2 \mathrm{H}_{\mathrm{ar}}\right) \mathrm{ppm}\right.$; ${ }^{13} \mathrm{C}-\mathrm{NMR}\left(\mathrm{CDCl}_{3} / \mathrm{TMS}\right): \delta=18.88\left(\mathrm{SCH}_{3}\right), 102.52$ (quart. C), 131.80, 132.32, $135.18\left(\mathrm{C}_{\mathrm{ar}}\right)$ ppm. [Dichloro(methylthio)-methyl] 4-chlorophenyl sulfone (12c): $100 \%$, colorless crystals, m.p. 74-74.5 ${ }^{\circ} \mathrm{C}, \mathrm{C}_{8} \mathrm{H}_{7} \mathrm{Cl}_{3} \mathrm{O}_{2} \mathrm{~S}_{2}$ (305.7), calc. C $31.44 \mathrm{H} 2.31$ found C $31.32 \mathrm{H}$ 2.47; IR (KBr): 1340, $1160\left(\mathrm{SO}_{2}\right) \mathrm{cm}^{-1} ;{ }^{1} \mathrm{H}-\mathrm{NMR}\left(\mathrm{CDCl}_{3} / \mathrm{TMS}\right)$ : $\delta=2.650\left(\mathrm{~s}, 3 \mathrm{H}, \mathrm{SCH}_{3}\right), 7.580\left(\mathrm{~d}, \mathrm{~J}=8.9 \mathrm{~Hz}, 2 \mathrm{H}_{\mathrm{ar}}\right), 8.021\left(\mathrm{~d}, \mathrm{~J}=8.6 \mathrm{~Hz}, 2 \mathrm{H}_{\mathrm{ar}}\right) \mathrm{ppm}$; ${ }^{13} \mathrm{C}-\mathrm{NMR}$ $\left(\mathrm{CDCl}_{3} / \mathrm{TMS}\right): \delta=18.94\left(\mathrm{SCH}_{3}\right), 102.53$ (quart. C), 128.91, 129.11, 129.25, 130.24, 133.65, $142.38\left(\mathrm{C}_{\mathrm{ar}}\right) \mathrm{ppm}$. [Dichloro-(4-methylphenylthio)-methyl] 4-methylphenyl sulfone $(\underline{\mathbf{1 2 e}}): 26 \%$, colorless crystals, m.p. $122{ }^{\circ} \mathrm{C}$, $\mathrm{C}_{15} \mathrm{H}_{14} \mathrm{Cl}_{2} \mathrm{O}_{2} \mathrm{~S}_{2}$ (361.3) $\mathrm{M}^{+}: \mathrm{m} / \mathrm{e}=361$; calc. C $49.86 \mathrm{H} 3.91$ found $\mathrm{C} 49.85 \mathrm{H} 3.87$; IR $(\mathrm{KBr}): 1340,1160\left(\mathrm{SO}_{2}\right)$ $\mathrm{cm}^{-1}$; ${ }^{1} \mathrm{H}-\mathrm{NMR}\left(\mathrm{CDCl}_{3} / \mathrm{TMS}\right): \delta=2.397\left(\mathrm{~s}, 3 \mathrm{H}, \mathrm{S}-\mathrm{ArCH}_{3}\right), 2.482\left(\mathrm{~s}, 3 \mathrm{H}, \mathrm{SO}_{2}-\mathrm{ArCH}_{3}\right), 7.233(\mathrm{~d}, \mathrm{~J}=8.1 \mathrm{~Hz}, 2$ $\mathrm{H}_{\mathrm{ar}}$ ), $7.383\left(\mathrm{~d}, \mathrm{~J}=8.2 \mathrm{~Hz}, 2 \mathrm{H}_{\mathrm{ar}}\right), 7.657\left(\mathrm{~d}, \mathrm{~J}=8.1 \mathrm{~Hz}, 2 \mathrm{H}_{\mathrm{ar}}\right), 7.982\left(\mathrm{~d}, \mathrm{~J}=8.3 \mathrm{~Hz}, 2 \mathrm{H}_{\mathrm{ar}}\right) \mathrm{ppm}$; ${ }^{13} \mathrm{C}-\mathrm{NMR}$ $\left(\mathrm{CDCl}_{3} / \mathrm{TMS}\right): \delta=21.47\left(\mathrm{~S}-\mathrm{ArCH}_{3}\right), 21.80\left(\mathrm{SO}_{2}-\mathrm{ArCH}_{3}\right), 103.26$ (quart. C), 125.06, 129.23, 129.40, 129.77, 132.46, 138.07, 142.11, $146.58\left(\mathrm{C}_{\mathrm{ar}}\right)$ ppm. 2,2-Dichloro-1,3-dithiolane-1,1-dioxide $(\mathbf{1 2 f}):$ 78\%, colorless crystals, m.p. $185^{\circ} \mathrm{C}, \mathrm{C}_{3} \mathrm{H}_{4} \mathrm{Cl}_{2} \mathrm{O}_{2} \mathrm{~S}_{2}(207.2)$, no elemental analysis available; IR (KBr): 1335, 1325, 1155,1140 $\left(\mathrm{SO}_{2}\right) \mathrm{cm}^{-1} ;{ }^{1} \mathrm{H}-\mathrm{NMR}\left(\mathrm{CDCl}_{3} / \mathrm{TMS}\right): \delta=3.377$ - $3.412\left(\mathrm{t}, \mathrm{J}=7 \mathrm{~Hz}, 2 \mathrm{H}, \mathrm{SCH}_{2}\right), 3.607-3.662(\mathrm{t}, \mathrm{J}=7 \mathrm{hz}, 2 \mathrm{H}$, $\left.\mathrm{SO}_{2} \mathrm{CH}_{2}\right)$ ppm; ${ }^{13} \mathrm{C}-\mathrm{NMR}\left(\mathrm{CDCl}_{3} / \mathrm{TMS}\right): \delta=97.55$ (C-2), 23.38 (C-4), 45.40 (C-5) ppm. 2,2-Dichloro1,3-dithiane-1,1-dioxide (12g): $96 \%$, colorless crystals, m.p. $140{ }^{\circ} \mathrm{C}_{2} \mathrm{C}_{4} \mathrm{H}_{6} \mathrm{Cl}_{2} \mathrm{O}_{2} \mathrm{~S}_{2}$ (221.1), no elemental analysis available; IR (KBr): 1335, 1155, $1145\left(\mathrm{SO}_{2}\right) \mathrm{cm}^{-1} ;{ }^{1} \mathrm{H}-\mathrm{NMR}\left(\mathrm{CDCl}_{3} / \mathrm{TMS}\right): \delta=2.670-2.728\left(\mathrm{~m}, 2 \mathrm{H}, \mathrm{CH}_{2}\right)$, $3.033\left(\mathrm{~s}, 2 \mathrm{H}, \mathrm{CH}_{2}\right), 3.580\left(\mathrm{~d}, \mathrm{~J}=5.2 \mathrm{~Hz}, 2 \mathrm{H}, \mathrm{CH}_{2}\right) \mathrm{ppm} ;{ }^{13} \mathrm{C}-\mathrm{NMR}\left(\mathrm{CDCl}_{3} / \mathrm{TMS}\right): \delta=98.69(\mathrm{C}-2), 31.18$ (C-4), 29.20 (C-5), 48.34 (C-6) ppm.

Arylsulfonyl-bromochloromethyl thioethers 13 (except 13d,e,h); general procedure: $5.6 \mathrm{~g}$ (35.3 mmol) bromine were added slowly to a mixture of $20 \mathrm{mmol}$ chlorosulfone $\underline{4}$ and $2 \mathrm{~g}(25.7 \mathrm{mmol})$ pyridine in $50 \mathrm{ml}$ chloroform with stirring at $0{ }^{\circ} \mathrm{C}$. Stirring was continued for $6 \mathrm{~h}$ at room temperature. $150 \mathrm{ml}$ water were added and the mixture was extracted three times with $50 \mathrm{ml}$ dichloromethane. The combined organic extracts were washed

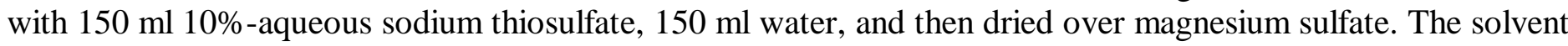
was removed i. vac. and the oily residue was crystallized from methanol. [Bromo-chloro-(methylthio)-methyl] phenyl sulfone (13a): $67 \%$, colorless crystals, m.p. $68^{\circ} \mathrm{C}, \mathrm{C}_{8} \mathrm{H}_{8} \mathrm{BrClO}_{2} \mathrm{~S}_{2}(314.7)$, no elemental analysis available; IR (KBr): $1325,1155\left(\mathrm{SO}_{2}\right) \mathrm{cm}^{-1} ;{ }^{1} \mathrm{H}-\mathrm{NMR}\left(\mathrm{CDCl}_{3} / \mathrm{TMS}\right): \delta=2.625\left(\mathrm{~s}, 3 \mathrm{H}, \mathrm{SCH}_{3}\right), 7.602\left(\mathrm{t}, \mathrm{J}=7.8 \mathrm{~Hz}, 2 \mathrm{H}_{\mathrm{ar}}\right)$, $7.757\left(\mathrm{t}, \mathrm{J}=6.2 \mathrm{~Hz}, 1 \mathrm{H}_{\mathrm{ar}}\right), 8.107\left(\mathrm{~d}, \mathrm{~J}=8.5 \mathrm{~Hz}, 2 \mathrm{H}_{\mathrm{ar}}\right)$ ppm; ${ }^{13} \mathrm{C}-\mathrm{NMR}\left(\mathrm{CDCl}_{3} / \mathrm{TMS}\right): \delta=20.53\left(\mathrm{SCH}_{3}\right), 90.72$ (quart. C), 128.48, 128.58, 131.37, 132.53, 132.78, $135.13\left(\mathrm{C}_{\mathrm{ar}}\right) \mathrm{ppm}$. [Bromo-chloro-(methylthio)-methyl] 4-methylphenyl sulfone (13b): $35 \%$, colorless crystals, m.p. $90{ }^{\circ} \mathrm{C}, \mathrm{C}_{9} \mathrm{H}_{10} \mathrm{BrClO}_{2} \mathrm{~S}_{2}$ (328.8), no elemental analysis available; IR (KBr) 1330, $1150\left(\mathrm{SO}_{2}\right) \mathrm{cm}^{-1} ;{ }^{1} \mathrm{H}-\mathrm{NMR}\left(\mathrm{CDCl}_{3} / \mathrm{TMS}\right): \delta=2.493\left(\mathrm{~s}, 3 \mathrm{H}, \mathrm{ArCH}_{3}\right), 2.622(\mathrm{~s}, 3 \mathrm{H}$, $\left.\mathrm{SCH}_{3}\right), 7.388\left(\mathrm{~d}, \mathrm{~J}=8.1 \mathrm{~Hz}, 2 \mathrm{H}_{\mathrm{ar}}\right), 7.984\left(\mathrm{~d}, \mathrm{~J}=8.4 \mathrm{~Hz}, 2 \mathrm{H}_{\mathrm{ar}}\right) \mathrm{ppm} ;{ }^{13} \mathrm{C}-\mathrm{NMR}\left(\mathrm{CDCl}_{3} / \mathrm{TMS}\right): \delta=21.82$ $\left(\mathrm{ArCH}_{3}\right), 20.54\left(\mathrm{SCH}_{3}\right), 90.98$ (quart. C), 128.27, 129.08, 129.32, 132.61, 132.84, $146.65\left(\mathrm{C}_{\mathrm{ar}}\right) \mathrm{ppm}$. [Bromo-chloro-(methylthio)-methyl] 4-chlorophenyl sulfone $(\underline{\mathbf{1 3 c}}): 52 \%$, colorless crystals, m.p. $71{ }^{\circ} \mathrm{C}$, $\mathrm{C}_{8} \mathrm{H}_{7} \mathrm{BrCl}_{2} \mathrm{O}_{2} \mathrm{~S}_{2}$ (350.1) calc. C $27.45 \mathrm{H} 2.02$ found $\mathrm{C} 27.40 \mathrm{H} 1.97$; IR (KBr): $1335,1150\left(\mathrm{SO}_{2}\right) \mathrm{cm}^{-1} ;{ }^{1} \mathrm{H}-\mathrm{NMR}$ $\left(\mathrm{CDCl}_{3} / \mathrm{TMS}\right): \delta=2.633\left(\mathrm{~s}, 3 \mathrm{H}, \mathrm{SCH}_{3}\right), 7.576\left(\mathrm{~d}, \mathrm{~J}=8.6 \mathrm{~Hz}, 2 \mathrm{H}_{\mathrm{ar}}\right), 8.041\left(\mathrm{~d}, \mathrm{~J}=8.6 \mathrm{~Hz}, 2 \mathrm{H}_{\mathrm{ar}}\right) \mathrm{ppm}$; ${ }^{13} \mathrm{C}-\mathrm{NMR}\left(\mathrm{CDCl}_{3} / \mathrm{TMS}\right): \delta=20.62\left(\mathrm{SCH}_{3}\right), 90.46$ (quart. C), 129.02, 129.11, 129.76, 133.65, 133.85, 142.34 $\left(\mathrm{C}_{\mathrm{ar}}\right)$ ppm. 2-Bromo-2-chloro-1,3-dithiolane-1,1-dioxide (13f): 76\%, colorless crystals, m.p. $204{ }^{\circ} \mathrm{C}$, $\mathrm{C}_{3} \mathrm{H}_{4} \mathrm{BrClO}_{2} \mathrm{~S}_{2}$ (250.7), no elemental analysis available; IR (KBr): 1350, 1155, $1145\left(\mathrm{SO}_{2}\right) \mathrm{cm}^{-1}$, ${ }^{1} \mathrm{H}-\mathrm{NMR}$ 
$\left(\mathrm{CDCl}_{3} / \mathrm{TMS}\right): \delta=3.344-3.458\left(\mathrm{~m}, 2 \mathrm{H}, \mathrm{SCH}_{2}\right), 3.581-3.658\left(\mathrm{~m}, 2 \mathrm{H}, \mathrm{SO}_{2} \mathrm{CH}_{2}\right)$ ppm; ${ }^{13} \mathrm{C}-\mathrm{NMR}$ $\left(\mathrm{CDCl}_{3} / \mathrm{TMS}\right): \delta=81.97$ (C-2), 24.57 (C-4), 44.09 (C-5) ppm. 2-Bromo-2-chloro-1,3-dithiane-1,1-dioxide (13g): $45 \%$, colorless crystals, m.p. $144{ }^{\circ} \mathrm{C}, \mathrm{C}_{4} \mathrm{H}_{6} \mathrm{BrClO}_{2} \mathrm{~S}_{2}$ (264.7), no elemental analysis available; IR (KBr): 1320, $1145\left(\mathrm{SO}_{2}\right) \mathrm{cm}^{-1} ;{ }^{1} \mathrm{H}-\mathrm{NMR}\left(\mathrm{CDCl}_{3} / \mathrm{TMS}\right): \delta=2.688-2.824\left(\mathrm{~m}, 3 \mathrm{H}, \mathrm{CH}_{2}\right), 3.163-3.222\left(\mathrm{~m}, 1 \mathrm{H}, \mathrm{CH}_{2}\right)$, 3.470 - $3.548\left(\mathrm{~m}, 1 \mathrm{H}, \mathrm{CH}_{2}\right), 3.684-3.740\left(\mathrm{~m}, 1 \mathrm{H}, \mathrm{CH}_{2}\right) \mathrm{ppm} ;{ }^{13} \mathrm{C}-\mathrm{NMR}\left(\mathrm{CDCl}_{3} / \mathrm{TMS}\right): \delta=85.93(\mathrm{C}-2), 31.78$ (C-4), 29.28 (C-5), 47.47 (C-6) ppm.

1-Methylthiovinyl 4-methylphenyl sulfone $(\underline{\mathbf{1 4}})^{49}$ via a one-pot procedure: A solution of $27.4 \mathrm{~g}(0.1 \mathrm{~mol})$ $\alpha$-[4-methylphenylsulfonyl] acetophenone ${ }^{38}$ in $100 \mathrm{ml}$ absol. acetonitrile was added dropwise under nitrogen to a stirred suspension of $6.6 \mathrm{~g}(0.22 \mathrm{~mol})$ 80-per cent sodium hydride in $11 \mathrm{labsol}$. acetonitrile at room temperature. After stirring for 2-3 h the evolution of hydrogen had ceased, $12.6 \mathrm{~g}(0.1 \mathrm{~mol}) \mathrm{S}$-methyl methanethiosulfonate in $20 \mathrm{ml}$ absol. acetonitrile were added and stirring was continued for additional $2 \mathrm{~h}$ at room temperature. During this period further evolution of hydrogen occurred, and sodium methanesulfinate precipitated at the same time. In a separate flask $20 \mathrm{~g}$ trioxane were monomerized to formaldehyde by heating with of $20 \mathrm{~g}$ of diphosphorus pentoxide under nitrogen to $180-200{ }^{\circ} \mathrm{C}$, and the formaldehyde-nitrogen gas stream was introduced into the reaction mixture causing additional precipitation of sodium benzoate ${ }^{50}$. After complete introduction of formaldehyde stirring was continued for $15 \mathrm{~min}$ at room temperature, the precipitated salts were removed by suction and washed with absol. acetonitrile. Most of the solvent was distilled off and the residue was poured into ice water. The resulting crystalline mixture of 1-methylthiovinyl 4-methylphenyl sulfone (14) and a small amount of paraformaldehyde which remained undissolved was recrystallized from ether, yielding $17.8 \mathrm{~g}(78 \%)$ colorless crystals, m.p. $74{ }^{\circ} \mathrm{C} ; \mathrm{C}_{10} \mathrm{H}_{12} \mathrm{O}_{2} \mathrm{~S}_{2}(228.3)$ calc. C $52.60 \mathrm{H} 5.30$ found C $52.4 \mathrm{H} 5.20 ; \mathrm{IR}(\mathrm{KBr}): 1310,1160\left(\mathrm{SO}_{2}\right)$ $\mathrm{cm}^{-1}$, no characteristical $\mathrm{C}=\mathrm{C}$ double bond absorption; ${ }^{1} \mathrm{H}-\mathrm{NMR}\left(\mathrm{CDCl}_{3} / \mathrm{TMS}\right): \delta=2.32\left(\mathrm{~s}, 3 \mathrm{H}, \mathrm{SCH}_{3}\right), 2.47(\mathrm{~s}$, $\left.3 \mathrm{H}, \mathrm{ArCH}_{3}\right), 5.70,6.58\left(2 \mathrm{~d}, \mathrm{~J}_{\mathrm{AB}}=2 \mathrm{~Hz}, 2 \mathrm{H}, \mathrm{CH}_{2}\right) \mathrm{ppm}^{51}$.

1.2-Bis(arylsulfonyl)-1.2-dimethylthio-cyclopropanes $\underline{\mathbf{Z}-16}$ : a) via Michael-addition and 1.3-S $\mathrm{S}_{\mathrm{N}} \mathrm{i}$-substitution (cyclopropanation). 1-Chloro-1-(4-chlorophenylsulfonyl)-1.3-dimethyl-thio-3-(4-methylphenylsulfonyl)propane (15a), mixture of diastereomers (m.d.): A solution of $650 \mathrm{mg}(2.8 \mathrm{mmol})$ chloro sulfonyl thioether $\mathbf{4 b}$ in $10 \mathrm{ml}$ THF and $30 \mathrm{ml}$ ether was dropped slowly to a vigorously stirred mixture of $770 \mathrm{mg}(2.8 \mathrm{mmol})$ vinyl sulfone $\underline{\mathbf{1 4}}$ and $10 \mathrm{mg}$ 18-crown-6 (as PTC-catalyst) in $10 \mathrm{ml}$ ether and $2 \mathrm{ml} \mathrm{50-per} \mathrm{cent} \mathrm{aqueous} \mathrm{potassium} \mathrm{hydroxide.}$ Stirring was continued for $12 \mathrm{~h}, 10 \mathrm{ml}$ water were added, and the phases were separated. After extraction of the aqueous phase twice with $10 \mathrm{ml}$ ether the combined organic phases were dried over magnesium sulfate and the solvent was removed i. vac.. The residue was taken up in $4 \mathrm{ml}$ methanol and crystallized at $-25{ }^{\circ} \mathrm{C}$. The resulting colorless crystals $(420 \mathrm{mg}$ ) consisted of a 4:1 mixture of 15a (m.d.) and cyclopropane $\underline{\mathbf{Z}-16 \mathbf{a}}$. Separation by column chromatography (column $70 \mathrm{~cm} \times 2.5 \mathrm{~cm}$, silica gel as stationary phase, eluent toluene-ethyl acetate 4:1, $\mathrm{R}_{\mathrm{F}}$ (15a) ca. $0.84, \mathrm{R}_{\mathrm{F}}(\underline{\mathbf{Z}-16 \mathbf{a})}$ ca. 0.52$)$ afforded $340 \mathrm{mg}(21 \%) \underline{15 a}$ and $80 \mathrm{mg}$ (4\%) 1-(4-chlorophenylsulfonyl)-1,2-dimethylthio-2-(4-methylphenylsulfonyl) cyclopropane (Z-16a); 15a (m.d.), m.p. $145{ }^{\circ} \mathrm{C}$, colorless crystals from i-propanol; $\mathrm{C}_{18} \mathrm{H}_{20} \mathrm{Cl}_{2} \mathrm{O}_{4} \mathrm{~S}_{4}$ (499.5) calc. C $43.28 \mathrm{H} 4.04$ found C $43.18 \mathrm{H} 3.91$; IR (KBr): 1329, 1314, 1304, 1290, 1283, $1148\left(\mathrm{SO}_{2}\right) \mathrm{cm}^{-1} ;{ }^{1} \mathrm{H}-\mathrm{NMR}\left(\mathrm{CDCl}_{3} / \mathrm{TMS}\right): \delta=2.34\left(\mathrm{~s}, 3 \mathrm{H}, \mathrm{SCH}_{3}\right), 2.47(\mathrm{~s}, 3 \mathrm{H}$, $\left.\mathrm{ArCH}_{3}\right), 2.52\left(\mathrm{~s}, 3 \mathrm{H}, \mathrm{SCH}_{3}\right), 3.06-3.20\left(\mathrm{~m}, 2 \mathrm{H}, \mathrm{CH}_{\mathrm{A}} \mathrm{H}_{\mathrm{B}}\right), 3.97\left(\mathrm{q}, \mathrm{J}_{\mathrm{AX}}+\mathrm{BX}=9.5 \mathrm{~Hz}, 1 \mathrm{H}, \mathrm{CH}_{\mathrm{X}}\right), 7.26-8.02(\mathrm{~m}$, $\left.8 \mathrm{H}, \mathrm{H}_{\mathrm{ar}}\right) \mathrm{ppm}^{48)}$; Z-16a, m.p. $197.5^{\circ} \mathrm{C}$, colorless crystals from methanol; $\mathrm{C}_{18} \mathrm{H}_{19} \mathrm{ClO}_{4} \mathrm{~S}_{4}(463.1)$, calc. C $46.69 \mathrm{H}$ 4.14 found C 46.52 H 3.93; IR (KBr): 3095, $3075\left(\mathrm{CH}_{\text {cyclopropyl }}\right), 1330,1320,1305,1158\left(\mathrm{SO}_{2}\right) \mathrm{cm}^{-1} ;{ }^{1} \mathrm{H}-\mathrm{NMR}$ $\left(\mathrm{CDCl}_{3} / \mathrm{TMS}\right): \delta=1.92\left(\mathrm{~d}, \mathrm{~J}=7.3 \mathrm{~Hz}, 1 \mathrm{H}_{\text {cyclopropyl }}\right), 2.12\left(\mathrm{~s}, 3 \mathrm{H}, \mathrm{SCH}_{3}\right), 2.18\left(\mathrm{~s}, 3 \mathrm{H}, \mathrm{SCH}_{3}\right), 2.46(\mathrm{~s}, 3 \mathrm{H}$, $\left.\mathrm{ArCH}_{3}\right), 3.27\left(\mathrm{~d}, \mathrm{~J}=7.3 \mathrm{~Hz}, 1 \mathrm{H}_{\text {cyclopropyl }}\right), 7.30-8.14\left(\mathrm{~m}, 8 \mathrm{H}, \mathrm{H}_{\mathrm{ar}}\right) \mathrm{ppm}^{48}$.

b) via one-pot carbene $\underline{\mathbf{3 b}}$ trapping with diazomethane: A solution of diazomethane (large excess) in ether had been prepared from $10 \mathrm{~g}(0.11 \mathrm{~mol}) \mathrm{N}$-nitrosomethyl urea according to usual conditions ${ }^{52}$ (Caution! Working with diazomethane and nitrosomethyl urea must ensue under suitable safety conditions!). $10 \mathrm{ml} 50 \%$ aqueous potassium hydroxide solution and $80 \mathrm{mg}(0.3 \mathrm{mmol}) 18$-crown- 6 were added under stirring. A solution of $2 \mathrm{~g}$ ( 8 $\mathrm{mmol}$ ) chloro sulfonyl thioether $\underline{\mathbf{4 b}}$ in $20 \mathrm{ml}$ THF and $60 \mathrm{ml}$ ether was dropped slowly into the stirred mixture at room temperature and stirring was continued for $12 \mathrm{~h}$ after addition. After this time excess diazomethane was destroyed by dropwise addition of hydrogen chloride solution in ether (decolorization). Ether was stripped off $\mathrm{i}$. vac., and the remaining solid-liquid mixture was extracted with chloroform. During concentration of the chloroform phase $350 \mathrm{mg}$ (20\%) 1,2-Bis(4-methylphenylsulfonyl)-1,2-dimethylthiocyclopropane ( crystallized and were collected by filtration. The remaining oil was a mixture of at least 8 products (TLC-analysis) from which the main component 1-chloro-1,3-bis(4-methylphenylsulfonyl)-1,3-dimethylthio-

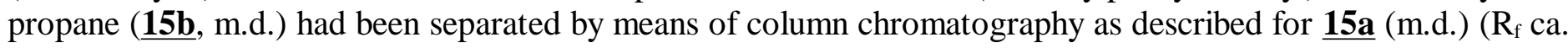
0.58), mollifying $>90^{\circ} \mathrm{C} ; \mathrm{C}_{19} \mathrm{H}_{23} \mathrm{ClO}_{4} \mathrm{~S}_{4}$ (479.1) calc. $\mathrm{C} 47.63 \mathrm{H} 4.84$ found $\mathrm{C} 47.82 \mathrm{H} \mathrm{4.70;} \mathrm{IR} \mathrm{(KBr):} \mathrm{1331,}$ 1328, 1302, 1292, $1146\left(\mathrm{SO}_{2}\right) \mathrm{cm}^{-1} ;{ }^{1} \mathrm{H}-\mathrm{NMR}\left(\mathrm{CDCl}_{3} / \mathrm{TMS}\right) \delta=2.33\left(\mathrm{~s}, 3 \mathrm{H}, \mathrm{SCH}_{3}\right), 2.47\left(\mathrm{~s}, 3 \mathrm{H}, \mathrm{ArCH}_{3}\right), 2.51$ 
(s, $\left.3 \mathrm{H}, \mathrm{SCH}_{3}\right), 2.54$ (s, $\left.3 \mathrm{H}, \mathrm{ArCH}_{3}\right), 3.15-3.19\left(\mathrm{~m}, 2 \mathrm{H}, \mathrm{CH}_{\mathrm{A}} \mathrm{H}_{\mathrm{B}}\right), 3.97$ (q, J = 9.5 Hz, $1 \mathrm{H}, \mathrm{CH}_{\mathrm{X}}$ ), $7.25-7.96$ $\left(\mathrm{m}, 8 \mathrm{H}, \mathrm{H}_{\mathrm{ar}}\right) \mathrm{ppm}^{48}$; Z-16b, m.p. $190-191{ }^{\circ} \mathrm{C}$, colorless crystals from methanol, $\mathrm{C}_{19} \mathrm{H}_{22} \mathrm{O}_{4} \mathrm{~S}_{4}(442.6)$ calc. $\mathrm{C}$ $51.55 \mathrm{H} 5.01 \mathrm{~S} 28.98$ found C $51.50 \mathrm{H} 4.97 \mathrm{~S} 29.43$; IR $(\mathrm{KBr}): 3020\left(\mathrm{CH}_{\text {cyclopropyl }}\right), 1325,1155\left(\mathrm{SO}_{2}\right) \mathrm{cm}^{-1}$; ${ }^{1} \mathrm{H}-\mathrm{NMR}\left(\mathrm{CDCl}_{3} / \mathrm{TMS}\right): \delta=1.901\left(1 \mathrm{H}, \mathrm{J}_{\mathrm{AB}}=7 \mathrm{~Hz}, \mathrm{CH}_{\text {B-cyclopropyl }}\right), 2.135\left(\mathrm{~s}, 6 \mathrm{H}, \mathrm{SCH}_{3}\right), 2.461\left(\mathrm{~s}, 6 \mathrm{H}, \mathrm{ArCH}_{3}\right)$, $3.274\left(1 \mathrm{H}, \mathrm{J}_{\mathrm{AB}}=7 \mathrm{~Hz}, \mathrm{CH}_{\text {A-cyclopropyl }}\right), 7.348$ (d, J = $\left.8 \mathrm{~Hz}, 4 \mathrm{H}_{\mathrm{ar} \text { meta }}\right), 7.979$ (d, J = $8 \mathrm{~Hz}, 4 \mathrm{H}_{\mathrm{ar} \text { ortho }}$ ) ppm; cf. computer plot from X-ray analysis of $\underline{\mathbf{Z}-\mathbf{1 6} \mathbf{b}}$, Fig. I.

3-Methylthio- and 5-methyl-3-methylthio pyrazolium hydrochlorides 18a, 18b. a) 3-(4-Methylphenylsulfonyl)3-methylthio-1-pyrazoline (17a): Usually prepared ethereal diazomethane solution was added in portions to a stirred solution of $2.28 \mathrm{~g}(10 \mathrm{mmol})$ methylthiovinyl sulfone $\mathbf{1 4}$ in $50 \mathrm{ml}$ absol. THF until the yellow color remained persistent and stirring was continued for $30 \mathrm{~min}$. Excess diazomethane was destroyed by addition of sufficient acetic acid and the solvent was removed below $40{ }^{\circ} \mathrm{C}$ i. vac.. The residue was recrystallized from dry diethylether yielding $2.4 \mathrm{~g}(89 \%) \underline{\mathbf{1 7 a}}$, colorless crystals, m.p. $101{ }^{\circ} \mathrm{C} ; \mathrm{C}_{11} \mathrm{H}_{14} \mathrm{~N}_{2} \mathrm{O}_{2} \mathrm{~S}_{2}$ calc. C $48.86 \mathrm{H} 5.22 \mathrm{~N}$ 10.36 found $\mathrm{C} 48.81 \mathrm{H} 5.19 \mathrm{~N}$ 10.39; IR $(\mathrm{KBr}): 1590(\mathrm{~N}=\mathrm{N}), 1300,1150\left(\mathrm{SO}_{2}\right) \mathrm{cm}^{-1},{ }^{1} \mathrm{H}-\mathrm{NMR}\left(\mathrm{CDCl}_{3} / \mathrm{TMS}\right): \delta$ $=1.85-2.85\left(\mathrm{~m}, 2 \mathrm{H}, \mathrm{CH}_{2}\right), 2.30\left(\mathrm{~s}, 3 \mathrm{H}, \mathrm{SCH}_{3}\right), 2.53\left(\mathrm{~s}, 3 \mathrm{H}, \mathrm{ArCH}_{3}\right), 4.76\left(\mathrm{t}, \mathrm{J}=7.5 \mathrm{~Hz}, 2 \mathrm{H}, \mathrm{NCH}_{2}\right), 7.7(\mathrm{q}$, $\mathrm{J}=8 \mathrm{~Hz}, 4 \mathrm{H}_{\mathrm{ar}}$ ) ppm. b) 3-Methylthio-pyrazolium hydrochloride (18a) via acid catalyzed aromatization of 17a: 1 $\mathrm{g}(3.7 \mathrm{mmol})$ pyrazoline $\mathbf{1 7 a}$ in $30 \mathrm{ml}$ ethanol and $1 \mathrm{ml}(10 \mathrm{mmol})$ conc. hydrochloric acid were refluxed for 30 min. The solvent was removed i. vac. and the resulting residue recrystallized from ethylacetate; $0.5 \mathrm{~g}(90 \%)$ light yellow crystals of 18a, m.p. 66-67 ${ }^{\circ} \mathrm{C}^{53} ; \mathrm{C}_{4} \mathrm{H}_{7} \mathrm{ClN}_{2} \mathrm{~S}$ (150.6) calc. C $31.89 \mathrm{H} 4.68 \mathrm{~N} 18.6$ found $\mathrm{C} 31.76 \mathrm{H} 4.69$ N 18.4; IR (KBr): 3520 - $3220(\mathrm{NH}), 1550(\mathrm{C}=\mathrm{N}) \mathrm{cm}^{-1} ;{ }^{1} \mathrm{H}-\mathrm{NMR}\left(\mathrm{CDCl}_{3} / \mathrm{TMS}\right): \delta=2.60\left(\mathrm{~s}, 3 \mathrm{H}, \mathrm{CH}_{3} \mathrm{~S}\right), 6.51$ $(\mathrm{s}, 1 \mathrm{H}, \mathrm{CH}), 8.01(\mathrm{~s}, 1 \mathrm{H}, \mathrm{CH}), 11.8-13.2(\mathrm{~m}, 2 \mathrm{H}, 2 \mathrm{NH})$ ppm. c) 5-Methyl-3-methylthio pyrazolium hydrochloride (18b): $2.28 \mathrm{~g}(10 \mathrm{mmol})$ methylthiovinyl sulfone $\underline{\mathbf{1 4}}$ were treated with ethereal diazoethane solution as described before with diazomethane. After the corresponding work-up pyrazoline 17b had already split off 4-toluene sulfinic acid in part, therefore, the residue was treated with $1 \mathrm{ml}(10 \mathrm{mmol})$ conc. hydrochloric acid as described before. The residue was crystallized firstly from ether/ethanol mixture and then from ethyl acetate/petroleum ether, $1.2 \mathrm{~g}$ (72\%) pale yellow crystals, m.p. 162-164 ${ }^{\circ} \mathrm{C}$ (dec.) (known as picrate $\left.{ }^{54}\right), \mathrm{C}_{5} \mathrm{H}_{9} \mathrm{ClN}_{2} \mathrm{~S}$ (164.7) calc. C 36.47, H 5.51 N 17.02 found C $36.79 \mathrm{H} 5.48 \mathrm{~N}$ 16.32; IR (KBr): 3160, $3000-2010\left(\mathrm{~N}^{+} \mathrm{H}\right), 1590(\mathrm{C}=\mathrm{N}) \mathrm{cm}^{-1} ;{ }^{1} \mathrm{H}-\mathrm{NMR}\left(\mathrm{CDCl}_{3} / \mathrm{TMS}\right): \delta=2.41\left(\mathrm{~s}, 3 \mathrm{H}, \mathrm{CH}_{3}\right), 2.63\left(\mathrm{~s}, 3 \mathrm{H}, \mathrm{SCH}_{3}\right), 6.43$ (s, 1 H, CH), 12.3 - 13.55 (m, 2 H, 2 NH) ppm. 4,5-Dimethyl-3-methylthio-pyrazole (21) and 4,5-Dimethyl-3(4-methylphenylsulfonyl)-pyrazole (22): Carbene $\underline{\mathbf{3 b}}$, generated from $2 \mathrm{~g}(8 \mathrm{mmol})$ chloro sulfone $\mathbf{4 b}$ under PTC-conditions equal to the trapping reaction with diazomethane (yielding $\underline{\mathbf{Z}-\mathbf{1 6} \mathbf{b}}$ ), was converted with diazoethane. During the removal of the ether solvent $80 \mathrm{mg}(4 \%)$ sulfonyl pyrazole 22 crystallized from the reaction mixture, pure enough to be washed only with pure ether for purification; colorless crystals, m.p. $230{ }^{\circ} \mathrm{C}$; $\mathrm{C}_{12} \mathrm{H}_{14} \mathrm{~N}_{2} \mathrm{O}_{2} \mathrm{~S}(250.3) ; \mathrm{MS}: \mathrm{m} / \mathrm{e}=250\left(\mathrm{M}^{+}\right)$; calc. C $57.57 \mathrm{H} 5.64 \mathrm{~N} 11.19$ found $\mathrm{C} 57.40 \mathrm{H} 5.56 \mathrm{~N} 11.00$; IR $(\mathrm{KBr}): 3295(\mathrm{NH}), 1598,1580(\mathrm{C}=\mathrm{N}), 1310,1295,1145\left(\mathrm{SO}_{2}\right) \mathrm{cm}^{-1} ;{ }^{1} \mathrm{H}-\mathrm{NMR}\left(\mathrm{CDCl}_{3} / \mathrm{TMS}\right): \delta=2.12(\mathrm{~s}, 3 \mathrm{H}$, $\left.\mathrm{CH}_{3}\right), 2.18\left(\mathrm{~s}, 3 \mathrm{H}, \mathrm{CH}_{3}\right), 2.43\left(\mathrm{~s}, 3 \mathrm{H}, \mathrm{ArCH}_{3}\right), 7.30-7.95\left(\mathrm{~m}, 4 \mathrm{H}_{\mathrm{ar}}\right), 13.1(\mathrm{~s}, 1 \mathrm{H}, \mathrm{NH}) \mathrm{ppm}$. The mother liquor was evaporated to dryness, the residue was treated with $1 \mathrm{ml}$ conc. hydrochloric acid in a sublimation apparatus. Sublimation $\left(95{ }^{\circ} \mathrm{C} / 0.18\right.$ Torr) yielded $1.1 \mathrm{~g} \mathbf{2 1} \times \mathrm{HCl}(77 \%)$, m.p. $133{ }^{\circ} \mathrm{C}$ after resublimation at 40-50 ${ }^{\circ} \mathrm{C} / 7.10^{-3}$ Torr; $\mathrm{C}_{6} \mathrm{H}_{11} \mathrm{ClN}_{2} \mathrm{~S}$ (178.7), MS: $\mathrm{m} / \mathrm{e}=178\left(\mathrm{M}^{+}\right)$and $142\left(\mathrm{M}^{+}-\mathrm{HCl}\right)$; calc. $\mathrm{C} 40.33 \mathrm{H} 6.21 \mathrm{~N}$ 15.68 found $\mathrm{C} 40.40 \mathrm{H} 6.15 \mathrm{~N}$ 15.40; IR $(\mathrm{KBr}): 2800-2600\left(\mathrm{NH}^{+}\right), 1573,1568(\mathrm{C}=\mathrm{N})$; ${ }^{1} \mathrm{H}-\mathrm{NMR}$ $\left(\mathrm{CDCl}_{3} / \mathrm{TMS}\right): \delta=2.02\left(\mathrm{~s}, 3 \mathrm{H}, \mathrm{CH}_{3}\right), 2.48\left(\mathrm{~s}, 3 \mathrm{H}, \mathrm{CH}_{3}\right), 2.72\left(\mathrm{~s}, 3 \mathrm{H}, \mathrm{SCH}_{3}\right), 14.48(\mathrm{~s}, 2 \mathrm{H}, \mathrm{NH}) \mathrm{ppm}$.

1-Arylsulfonyl-1-organylthio-2-alkoxy resp. 2-aryl-cyclopropanes $\underline{\mathbf{7}}$ via cyclopropanation of enol ethers or styrenes $\underline{\mathbf{6}}$ with carbenes $\underline{\mathbf{3}}$ under PTC-conditions; general procedure: A two- phase mixture of $30 \mathrm{mmol}$ olefin and 10-80 mg (1-6 mol per cent) 18-crown-6 in $100 \mathrm{ml}$ ether and $10 \mathrm{ml} 50$-per cent potassium hydroxide was prepared. Under vigorous stirring a solution of $5 \mathrm{mmol}$ of the corresponding $\alpha$-chloro- $\alpha$-sulfonyl thioether $\underline{4}$ dissolved in $10 \mathrm{ml}$ THF and $40 \mathrm{ml}$ ether was dropped into the two-phase mixture and the reaction was continuously monitored by TLC. Volatile olefins were converted at $0{ }^{\circ} \mathrm{C}$, while in other cases reactions were carried out at room temperature; most reactions were finished within $24 \mathrm{~h}$, in some cases stirring had to be maintained up to $7 \mathrm{~d}$. After $\underline{4}$ had disappeared, $100 \mathrm{ml}$ water was added, the phases were separated, and the aqueous phase was extracted twice with $100 \mathrm{ml}$ ether. The combined ether phases were dried over $\mathrm{MgSO}_{4}$, solvent was distilled off, and in cases in which styrenes had been applied as trapping agents, the residue was evaporated i. vac. at $30{ }^{\circ} \mathrm{C}$. In most cases crystallization and recrystallization of the residue from methanol was sufficient to get pure cyclopropanes $\underline{\mathbf{7}}$. Obstinate impurities could be removed via column chromatography on silica gel. 
Table III: Physical data of obtained cyclopropanes $\underline{\mathbf{7 a}-\mathbf{x}}$

\begin{tabular}{|c|c|c|}
\hline -cyclopropane $\underline{7}$ & & $\operatorname{NMR}\left(\mathrm{CDCl}_{3} / \mathrm{TMS}\right), \delta(\mathrm{ppm})$ \\
\hline $\begin{array}{l}\text { a r-1-(4-methylphenylsulfonyl)-t-2- } \\
\text { methoxy-t-1-methylthio- } \\
\qquad \mathrm{C}_{12} \mathrm{H}_{16} \mathrm{O}_{3} \mathrm{~S}_{2} \\
\quad(272.4) \\
\text { m.p. }\left(\mathrm{CH}_{3} \mathrm{OH}\right): 83^{\circ} \mathrm{C}\end{array}$ & $\begin{array}{l}\text { calc. C } 52.92 \text { H } 5.92 \\
\text { found C } 52.98 \text { H } 5.72 \\
\\
\text { IR }(\mathrm{KBr})\left(\mathrm{cm}^{-1}\right): \\
1300,1150\left(\mathrm{SO}_{2}\right)\end{array}$ & $\begin{array}{l}1 \mathrm{H}: 1.506\left(\mathrm{dd}, \mathrm{J}=5.1 /-7 \mathrm{~Hz}, 1 \mathrm{H}, \mathrm{H}_{\mathrm{B}}\right), 2.153(\mathrm{dd}, \mathrm{J}=7.6 /-7 \mathrm{~Hz}, 1 \mathrm{H}, \\
\left.\mathrm{H}_{\mathrm{A}}\right), 2.201\left(\mathrm{~s}, 3 \mathrm{H}, \mathrm{SCH}_{3}\right), 2.459\left(\mathrm{~s}, 3 \mathrm{H}, \mathrm{ArCH}_{3}\right), 3.271\left(\mathrm{~s}, 3 \mathrm{H}, \mathrm{OCH}_{3}\right), \\
4.158\left(\mathrm{dd}, \mathrm{J}=5.1 / 7.6 \mathrm{~Hz}, 1 \mathrm{H}, \mathrm{H}_{\mathrm{x}}\right), 7.363,7.856(\mathrm{~d}, \mathrm{~J}=8.2 \mathrm{~Hz}, 4 \\
\left.\mathrm{H}_{\mathrm{ar}}\right) .{ }^{13} \mathrm{C}: 16.28\left(\mathrm{SCH}_{3}\right), 21.65\left(\mathrm{ArCH}_{3}\right), 22.23(\mathrm{C}-3), 50.92(\mathrm{C}-1), \\
59.44\left(\mathrm{OCH}_{3}\right), 66.18(\mathrm{C}-2), 129.39,129.40,134.66,144.72\left(\mathrm{C}_{\mathrm{ar}}\right) .\end{array}$ \\
\hline $\begin{array}{l}\underline{\mathbf{b}} \text { r-1-(4-chlorophenylsulfonyl)-t-2- } \\
\text { methoxy-t-1-methylthio- } \\
\qquad \mathrm{C}_{11} \mathrm{H}_{13} \mathrm{ClO}_{3} \mathrm{~S}_{2} \\
(292.8) \\
\text { m.p. }\left(\mathrm{CH}_{3} \mathrm{OH}\right): 78{ }^{\circ} \mathrm{C}\end{array}$ & $\begin{array}{c}\text { calc. C } 45.12 \mathrm{H} 4.47 \\
\text { found C } 44.91 \mathrm{H} 4.41 \\
\quad \mathrm{IR}(\mathrm{KBr})\left(\mathrm{cm}^{-1}\right) \text { : } \\
1318,1302,1158 \\
1150\left(\mathrm{SO}_{2}\right)\end{array}$ & $\begin{array}{l}{ }^{1} \mathrm{H}: 1.54\left(\mathrm{dd}, \mathrm{J}=5.5 /-7.5 \mathrm{~Hz}, 1 \mathrm{H}, \mathrm{H}_{\mathrm{B}}\right), 2.17\left(\mathrm{t}, \mathrm{J}=7.5 \mathrm{~Hz}, 1 \mathrm{H}, \mathrm{H}_{\mathrm{A}}\right), \\
2.26(\mathrm{~s}, 3 \mathrm{H}, \mathrm{SCH}), 3.31\left(\mathrm{~s}, 3 \mathrm{H}, \mathrm{OCH}_{3}\right), 4.20(\mathrm{dd}, \mathrm{J}=5.5 / 7.5 \mathrm{~Hz}, 1 \mathrm{H}, \\
\left.\mathrm{H}_{\mathrm{x}}\right), 7.53-8.08\left(\mathrm{~m}, 4 \mathrm{H}_{\mathrm{ar}}\right)\end{array}$ \\
\hline $\begin{array}{l}\text { c r-1-(4-methylphenylsulfonyl)-t-2- } \\
\text { ethoxy-t-1-methylthio- } \\
\qquad \mathrm{C}_{13} \mathrm{H}_{18} \mathrm{O}_{3} \mathrm{~S}_{2} \\
\quad(286.4) \\
\text { m.p. }\left(\mathrm{CH}_{3} \mathrm{OH}\right): 80{ }^{\circ} \mathrm{C}\end{array}$ & $\begin{array}{l}\text { calc. C } 54.52 \mathrm{H} 6.33 \\
\text { found C } 54.50 \mathrm{H} 6.19 \\
\\
\quad \mathrm{IR}(\mathrm{KBr})\left(\mathrm{cm}^{-1}\right) \text { : } \\
1300,1285,1150 \\
\qquad\left(\mathrm{SO}_{2}\right)\end{array}$ & $\begin{array}{l}1 \mathrm{H}: 1.164\left(\mathrm{t}, \mathrm{J}=7 \mathrm{~Hz}, 3 \mathrm{H}, \mathrm{CH}_{3}\right), 1.517\left(\mathrm{dd}, \mathrm{J}=5.2 /-6.9 \mathrm{~Hz}, 1 \mathrm{H}, \mathrm{H}_{\mathrm{B}}\right), \\
2.163\left(\mathrm{dd}, \mathrm{J}=7.8 /-6.9 \mathrm{~Hz}, 1 \mathrm{H}, \mathrm{H}_{\mathrm{A}}\right), 2.186\left(\mathrm{~s}, 3 \mathrm{H}, \mathrm{SCH}_{3}\right), 2.460(\mathrm{~s}, \\
\left.3 \mathrm{H}, \mathrm{ArCH}_{3}\right), 3.281,3.504\left(\mathrm{qd}, \mathrm{J}=7 /-9.3 \mathrm{~Hz}, 2 \mathrm{H}, \mathrm{OCH}_{2}\right), 4.206(\mathrm{dd}, \mathrm{J} \\
\left.=5.2 / 7.8 \mathrm{~Hz}, 1 \mathrm{H}, \mathrm{H}_{\mathrm{x}}\right), 7.360,7.850\left(\mathrm{~d}, \mathrm{~J}=8.2 \mathrm{~Hz}, 4 \mathrm{H}_{\mathrm{ar}}\right) .{ }^{\mathbf{1 3}} \mathrm{C}: 14.70 \\
\left(\mathrm{CH}_{3}\right), 16.26\left(\mathrm{SCH}_{3}\right), 21.65\left(\mathrm{ArCH}_{3}\right), 22.23(\mathrm{C}-3), 51.03(\mathrm{C}-1), 64.70 \\
(\mathrm{C}-2), 67.78\left(\mathrm{OCH}_{2}\right), 129.39,134.86,144.65\left(\mathrm{C}_{\mathrm{ar}}\right) .\end{array}$ \\
\hline $\begin{array}{l}\mathbf{d} \mathbf{r} \text {-1-(4-chlorophenylsulfonyl)-t-2- } \\
\text { ethoxy-t-1-methylthio- } \\
\qquad \mathrm{C}_{12} \mathrm{H}_{15} \mathrm{ClO}_{3} \mathrm{~S}_{2} \\
\qquad(306.8) \\
\text { m.p. }\left(\mathrm{CH}_{3} \mathrm{OH}\right): 60^{\circ} \mathrm{C}\end{array}$ & $\begin{array}{l}\text { calc. C } 46.98 \mathrm{H} 4.93 \\
\text { found } \mathrm{C} 46.79 \mathrm{H} 4.85 \\
\\
\mathrm{IR}(\mathrm{KBr})\left(\mathrm{cm}^{-1}\right): \\
1300,1310,1155 \\
\left(\mathrm{SO}_{2}\right)\end{array}$ & $\begin{array}{l}{ }^{1} \mathbf{H}: 1.172\left(\mathrm{t}, \mathrm{J}=7.1 \mathrm{~Hz}, 3 \mathrm{H}, \mathrm{CH}_{3}\right), 1.549\left(\mathrm{dd}, \mathrm{J}=7.8 /-7 \mathrm{~Hz}, 1 \mathrm{H}, \mathrm{H}_{\mathrm{B}}\right), \\
2.168\left(\mathrm{dd}, \mathrm{J}=5.2 /-7 \mathrm{~Hz}, 1 \mathrm{H}, \mathrm{H}_{\mathrm{A}}\right), 2.231\left(\mathrm{~s}, 3 \mathrm{H}, \mathrm{SCH}_{3}\right), 3.269,3.504 \\
\left(\mathrm{qd}, \mathrm{J}=7.1 /-9.3 \mathrm{~Hz}, 1 \mathrm{H}, \mathrm{OCH}_{2}\right), 4.192\left(\mathrm{dd}, \mathrm{J}=5.2 / 7.8 \mathrm{~Hz}, 1 \mathrm{H}, \mathrm{H}_{\mathrm{x}}\right), \\
7.545,7.911\left(\mathrm{~d}, \mathrm{~J}=8.7 \mathrm{~Hz}, 4 \mathrm{H}_{\mathrm{ar}}\right){ }^{13} \mathrm{C}: 14.67\left(\mathrm{CH}_{3}\right), 16.27\left(\mathrm{SCH}_{3}\right), \text {, } \\
22.26(\mathrm{C}-3), 50.92(\mathrm{C}-1), 64.73(\mathrm{C}-2), 67.82\left(\mathrm{OCH}_{2}\right), 129.05, \\
130.75,136.21,140.44\left(\mathrm{C}_{\mathrm{ar}}\right) .\end{array}$ \\
\hline $\begin{array}{l}\text { e r-1-(4-methylphenylsulfonyl)-t-2- } \\
\text { butoxy-t-1-methylthio- } \\
\qquad \mathrm{C}_{15} \mathrm{H}_{22} \mathrm{O}_{3} \mathrm{~S}_{2} \\
\quad(314.5) \\
\text { m.p. }\left(\mathrm{CH}_{3} \mathrm{OH}\right): 60{ }^{\circ} \mathrm{C}\end{array}$ & $\begin{array}{l}\text { calc. C } 57.29 \text { H } 7.05 \\
\text { found C } 57.28 \text { H } 6.92 \\
\\
\text { IR }(\mathrm{KBr})\left(\mathrm{cm}^{-1}\right): \\
1290,1300,1155 \\
\left(\mathrm{SO}_{2}\right)\end{array}$ & $\begin{array}{l}{ }^{1} \mathrm{H}: 0.864\left(\mathrm{t}, \mathrm{J}=7.4 \mathrm{~Hz}, 3 \mathrm{H}, \mathrm{CH}_{3}\right), 1.309\left(\mathrm{~m}, 2 \mathrm{H}, \mathrm{CH}_{2}-\mathrm{Me}\right), 1.494(\mathrm{~m}, \\
\left.2 \mathrm{H}, \mathrm{CH}_{2} \mathrm{CH}_{2} \mathrm{O}\right), 1.511\left(\mathrm{dd}, \mathrm{J}=5.2 /-6.8 \mathrm{~Hz}, 1 \mathrm{H}, \mathrm{H}_{\mathrm{B}}\right), 2.153(\mathrm{dd}, \mathrm{J}= \\
\left.7.6 /-7 \mathrm{~Hz}, 1 \mathrm{H}, \mathrm{H}_{\mathrm{A}}\right), 2.183\left(\mathrm{~s}, 3 \mathrm{H}, \mathrm{SCH}_{3}\right), 3.229,3.408(\mathrm{td}, \mathrm{J}= \\
\left.6.6 /-9.4 \mathrm{~Hz}, 2 \mathrm{H}, \mathrm{OCH}_{2}\right), 4.192\left(\mathrm{dd}, \mathrm{J}=5.2 / 7.6 \mathrm{~Hz}, 1 \mathrm{H}, \mathrm{H}_{\mathrm{x}}\right), 7.355, \\
7.848\left(\mathrm{~d}, \mathrm{~J}=8.3 \mathrm{~Hz}, 4 \mathrm{H}_{\mathrm{ar}}\right) .{ }^{13} \mathrm{C}: 13.68\left(\mathrm{CH}_{2}\right), 16.25\left(\mathrm{SCH}_{3}\right), 19.11 \\
\left(\mathrm{CH}_{2} \mathrm{Me}\right), 21.60\left(\mathrm{ArCH}_{3}\right), 22.29(\mathrm{C}-3), 31.24\left(\mathrm{CH}_{2} \mathrm{CH}_{2} \mathrm{O}\right), 51.06 \\
(\mathrm{C}-1), 64.86(\mathrm{C}-2), 72.09\left(\mathrm{OCH}_{2}\right), 129.34,134.95,144.59\left(\mathrm{C}_{\mathrm{ar}}\right) .\end{array}$ \\
\hline $\begin{array}{l}\text { f r-1-(4-chlorophenylsulfonyl)-t-2- } \\
\text { butoxy-t-1-methylthio- } \\
\qquad \begin{array}{l}\mathrm{C}_{14} \mathrm{H}_{19} \mathrm{ClO}_{3} \mathrm{~S}_{2} \\
(334.9) \\
\text { m.p. }\left(\mathrm{CH}_{3} \mathrm{OH}\right): 50{ }^{\circ} \mathrm{C}\end{array}\end{array}$ & $\begin{array}{l}\text { calc. C } 50.21 \mathrm{H} 5.72 \\
\text { found C } 50.15 \mathrm{H} 5.64 \\
\\
\quad \mathrm{IR}(\mathrm{KBr})\left(\mathrm{cm}^{-1}\right) \text { : } \\
1310,1155\left(\mathrm{SO}_{2}\right)\end{array}$ & $\begin{array}{l}1 \mathrm{H}: 0.872\left(\mathrm{t}, \mathrm{J}=7.3 \mathrm{~Hz}, 3 \mathrm{H}, \mathrm{CH}_{3}\right), 1.318\left(\mathrm{~m}, 2 \mathrm{H}, \mathrm{CH}_{2} \mathrm{CH}_{3}\right), 1.499 \\
\left(\mathrm{~m}, 2 \mathrm{H}, \mathrm{CH}_{2} \mathrm{CH}_{2} \mathrm{O}\right), 1.540\left(\mathrm{dd}, \mathrm{J}=5.3 /-7,1 \mathrm{H}, \mathrm{H}_{\mathrm{B}}\right), 2.157(\mathrm{dd}, \mathrm{J}= \\
\left.7.8 /-7 \mathrm{~Hz}, 1 \mathrm{H}, \mathrm{H}_{\mathrm{A}}\right), 2.229\left(\mathrm{~s}, 3 \mathrm{H}, \mathrm{SCH}_{3}\right), 3.219-3.412,(\mathrm{td}, \mathrm{J}= \\
\left.6.6 /-9.3 \mathrm{~Hz}, 2 \mathrm{H}, \mathrm{OCH})_{2}\right), 4.180\left(\mathrm{dd}, \mathrm{J}=5.3 / 7.8 \mathrm{~Hz}, 1 \mathrm{H}, \mathrm{H}_{\mathrm{x}}\right), 7.543, \\
7.911\left(\mathrm{~d}, \mathrm{~J}=8.8 \mathrm{~Hz}, 4 \mathrm{H}_{\mathrm{ar}}\right){ }^{13} \mathrm{C}: 13.68\left(\mathrm{CH}_{3}\right), 16.31\left(\mathrm{SCH}_{3}\right), 19.11 \\
\left(\mathrm{CH}_{2} \mathrm{Me}\right), 22.36(\mathrm{C}-3), 31.24\left(\mathrm{CH}_{2} \mathrm{CH}_{2} \mathrm{O}\right), 50.97(\mathrm{C}-1), 64.92(\mathrm{C}-2), \\
72.18\left(\mathrm{OCH}_{2}\right), 129.03,130.76,136.29,140.44\left(\mathrm{C}_{\mathrm{ar}}\right) .\end{array}$ \\
\hline $\begin{array}{l}\text { g r-1-phenylsulfonyl-t-3-ethylene- } \\
\text { t-2-oxy-t-1-methylthio-s5 } \\
\qquad \begin{array}{c}\mathrm{C}_{12} \mathrm{H}_{14} \mathrm{O}_{3} \mathrm{~S}_{2} \\
(270.4)\end{array} \\
\text { m.p. }\left(\mathrm{CH}_{3} \mathrm{OH}\right): 100-102{ }^{\circ} \mathrm{C} \\
\end{array}$ & $\begin{array}{l}\text { calc. C } 53.31 \text { H } 5.22 \\
\text { found C } 53.10 \text { H } 5.11\end{array}$ & $\begin{array}{l}{ }^{1} \mathrm{H}: 2.084-2.142\left(\mathrm{~m}, 1 \mathrm{H}, \mathrm{CH}_{2}\right), 2.154\left(\mathrm{~s}, 3 \mathrm{H}, \mathrm{SCH}_{3}\right), 2.393(\mathrm{~m}, 1 \mathrm{H}, \\
\left.\mathrm{CH}_{2}\right), 2.871\left(\mathrm{t}, \mathrm{J}=6.5 \mathrm{~Hz}, 1 \mathrm{H}, \mathrm{CHCH}_{2}\right), 4.136\left(\mathrm{~m}, 1 \mathrm{H}, \mathrm{OCH}_{2}\right), 4.210 \\
\left(\mathrm{~m}, 1 \mathrm{H}, \mathrm{CH}_{2} \mathrm{O}\right), 4.874(\mathrm{~d}, \mathrm{~J}=6.2 \mathrm{~Hz}, 1 \mathrm{H}, \mathrm{OCH}), 7.553(\mathrm{t}, \mathrm{J}=7.3 \mathrm{~Hz}, \\
\left.2 \mathrm{H}_{\mathrm{ar}}\right), 7.648\left(\mathrm{t}, \mathrm{J}=7.7 \mathrm{~Hz}, 1 \mathrm{H}_{\mathrm{ar}}\right), 7.956\left(\mathrm{~d}, \mathrm{~J}=8.3 \mathrm{~Hz}, 2 \mathrm{H}_{\mathrm{ar}}\right) .{ }^{13} \mathrm{C}: \\
15.38\left(\mathrm{SCH}_{3}\right), 26.41(\mathrm{C}-4), 32.44(\mathrm{C}-5), 56.15(\mathrm{C}-6), 69.62(\mathrm{C}-1), \\
74.30(\mathrm{C}-3), 128.76,129.30,133.54,138.23\left(\mathrm{C}_{\mathrm{ar}}\right) .\end{array}$ \\
\hline $\begin{array}{l}\text { h r-1-(4-methylphenylsulfonyl)-t-3- } \\
\text { ethylene-t-2-oxy-t-1-methylthio- }-{ }^{55} \\
\qquad \mathrm{C}_{{ }_{13} \mathrm{H}_{16} \mathrm{O}_{3} \mathrm{~S}_{2}} \\
\quad(284.4) \\
\text { m.p. }\left(\mathrm{CH}_{3} \mathrm{OH}\right): 85^{\circ} \mathrm{C}\end{array}$ & $\begin{array}{l}\text { calc. C } 54.91 \mathrm{H} 5.76 \\
\text { found C } 54.89 \mathrm{H} 5.63 \\
\\
\begin{array}{c}\text { IR }(\mathrm{KBr})\left(\mathrm{cm}^{-1}\right): \\
1295,1280,1150 \\
\left(\mathrm{SO}_{2}\right)\end{array}\end{array}$ & 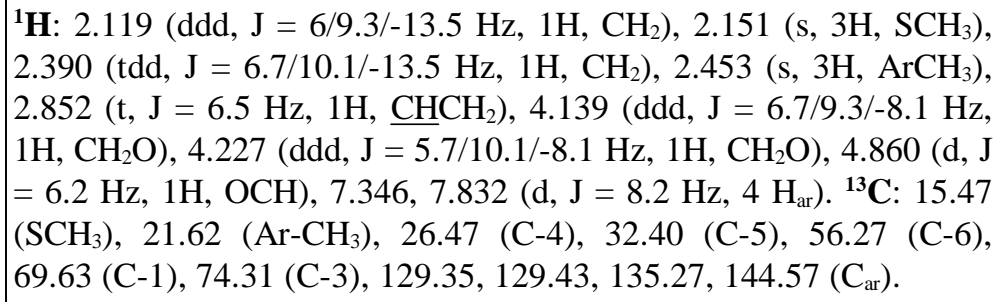 \\
\hline
\end{tabular}


i r-1-(4-chlorophenylsulfonyl)-t-3ethylene-t-2-oxy-t-1-methylthio- ${ }^{55}$

calc. C $47.29 \mathrm{H} 4.30$ found $\mathrm{C} 47.09 \mathrm{H} 4.19$

$\mathrm{C}_{12} \mathrm{H}_{13} \mathrm{ClO}_{3} \mathrm{~S}_{2}$ (304.8)

\begin{tabular}{l|l} 
& $\left(\mathrm{SO}_{2}\right)$ \\
m.p. $\left(\mathrm{CH}_{3} \mathrm{OH}\right): 112{ }^{\circ} \mathrm{C}$ & \\
\hline
\end{tabular}

\begin{tabular}{|l|l|}
\hline \multicolumn{1}{|c|}{-cyclopropane $\underline{7}$} & \\
\hline $\begin{array}{l}\text { j r-1-(4-methylphenylsulfonyl)-t-1- } \\
\text { (4-methylphenylthio)-t-3-propylene- } \\
\text { t-2-oxy-56 }\end{array}$ & $\begin{array}{l}\text { calc. C 64.17 H 5.88 } \\
\text { found C 64.01 H 5.85 }\end{array}$ \\
\hline
\end{tabular}
t-2-oxy- ${ }^{56}$

$$
\mathrm{C}_{20} \mathrm{H}_{22} \mathrm{O}_{3} \mathrm{~S}_{2}
$$$$
\text { (374.5) }
$$

m.p. $\left(\mathrm{CH}_{3} \mathrm{OH}\right): 133{ }^{\circ} \mathrm{C}$

$\underline{\text { k }}$ r-1-(4-methylphenylsulfonyl)-t-1methylthio-t-2-phenyl-

$$
\mathrm{C}_{17} \mathrm{H}_{18} \mathrm{O}_{2} \mathrm{~S}_{2}
$$

(318.5)

m.p. $\left(\mathrm{CH}_{3} \mathrm{OH}\right): 128^{\circ} \mathrm{C}$

I r-1-(4-methylphenylsulfonyl)-t-2(4-methoxyphenyl-t-1-methylthio-

$\mathrm{C}_{18} \mathrm{H}_{20} \mathrm{O}_{3} \mathrm{~S}_{2}$

(348.5)

m.p. $\left(\mathrm{CH}_{3} \mathrm{OH}\right): 120{ }^{\circ} \mathrm{C}$

$\underline{\mathbf{m}}$ r-1-(4-methylphenylsulfonyl)-t1-(4-methylphenylthio)-t-2-phenyl$\mathrm{C}_{23} \mathrm{H}_{22} \mathrm{O}_{2} \mathrm{~S}_{2}$ (394.6)

m.p. $\left(\mathrm{CH}_{3} \mathrm{OH}\right): 105^{\circ} \mathrm{C}$

n r-1-(4-methylphenylsulfonyl)-t-2(4-methoxyphenyl)-t-1-(4-methylphenylthio)-

$$
\mathrm{C}_{24} \mathrm{H}_{24} \mathrm{O}_{3} \mathrm{~S}_{2}
$$

(424.6)

m.p. $\left(\mathrm{CH}_{3} \mathrm{OH}\right): 148^{\circ} \mathrm{C}$

o r-1-(4-methylphenylsulfonyl)-t-2-, calc. C 50.33 H 4.94 t-3-methylendioxy-t-1-methylthio- ${ }^{57}$ found C $50.51 \mathrm{H} 4.93$ $\mathrm{C}_{12} \mathrm{H}_{14} \mathrm{O}_{4} \mathrm{~S}_{2}$ (286.4)

m.p. $\left(\mathrm{CH}_{3} \mathrm{OH}\right): 108^{\circ} \mathrm{C}$
$\mathrm{M}^{+}$at $\mathrm{m} / \mathrm{z} 374$

calc. C 64.15 H 5.66 found $\mathrm{C} 64.11 \mathrm{H} 5.76$

$\mathrm{M}^{+}$at $\mathrm{m} / \mathrm{z} 318$

calc. C62.07 H 5.75

found C $61.60 \mathrm{H} 5.67$

$\mathrm{M}^{+}$at $\mathrm{m} / \mathrm{z} 348$

calc. C 70.05 H 5.58

found $\mathrm{C} 70.06 \mathrm{H} 5.57$

$\mathrm{M}^{+}$at $\mathrm{m} / \mathrm{z} 394$

calc. C 67.92 H 5.66 found C $67.60 \mathrm{H} 5.56$

$\mathrm{M}^{+}$at $\mathrm{m} / \mathrm{z} 424$
$\operatorname{IR}(\mathrm{KBr})\left(\mathrm{cm}^{-1}\right)$ : $1300,1140\left(\mathrm{SO}_{2}\right)$
${ }^{1} \mathbf{H}: 2.117$ (ddd, J = 5.7/9.3/-13.2 Hz, $\left.1 \mathrm{H}, \mathrm{CH}_{2}\right), 2.200\left(\mathrm{~s}, 3 \mathrm{H}, \mathrm{SCH}_{3}\right)$, 2.402 (dddd, J = 6.7/6.7/10.1/-13.2 Hz, 1H, $\mathrm{CH}_{2}$ ), $2.837(\mathrm{t}, \mathrm{J}=6.3$ $\left.\mathrm{Hz}, 1 \mathrm{H}, \underline{\mathrm{CHCH}_{2}}\right), 4.149\left(\mathrm{ddd}, \mathrm{J}=6.7 / 9.3 /-8.1 \mathrm{~Hz}, 1 \mathrm{H}, \mathrm{CH}_{2} \mathrm{O}\right), 4.240$ $\left(\mathrm{ddd}, \mathrm{J}=5.7 / 10.1 /-8.1 \mathrm{~Hz}, 1 \mathrm{H}, \mathrm{CH}_{2} \mathrm{O}\right), 4.875(\mathrm{~d}, \mathrm{~J}=6.2 \mathrm{~Hz}, 1 \mathrm{H}$, $\mathrm{OCH}), 7.535,7.892\left(\mathrm{~d}, \mathrm{~J}=8.4 \mathrm{~Hz}, 4 \mathrm{H}_{\mathrm{ar}}\right) \cdot{ }^{13} \mathrm{C}: 15.63\left(\mathrm{SCH}_{3}\right), 26.47$ (C-4), 32.76 (C-5), 56.16 (C-6), 69.70 (C-1), 74.41 (C-3), 129.15, $130.80,136.64,140.45\left(\mathrm{C}_{\mathrm{ar}}\right)$.

$\mathrm{NMR}\left(\mathrm{CDCl}_{3} / \mathrm{TMS}\right), \delta(\mathrm{ppm})$

${ }^{1} \mathrm{H}: 1.92-2.19\left(\mathrm{~m}, 2 \mathrm{H}, \mathrm{C}^{4} \mathrm{H}_{2}\right), 2.288\left(\mathrm{~s}, 3 \mathrm{H}, \mathrm{CH}_{3} \mathrm{C}_{6} \mathrm{H}_{4} \mathrm{~S}\right), 2.389$ (s, 3H, $\left.\mathrm{CH}_{3} \mathrm{C}_{6} \mathrm{H}_{4} \mathrm{SO}_{2}\right), 2.667-2.817\left(\mathrm{~m}, 2 \mathrm{H}, \mathrm{C}^{5} \mathrm{H}_{2}\right), 3.449-3.73(\mathrm{~m}, 2 \mathrm{H}$, $\left.\mathrm{C}^{3} \mathrm{H}_{2}\right), 3.75$ - $3.85\left(\mathrm{~m}, 1 \mathrm{H}, \mathrm{C}^{6} \mathrm{H}\right), 4.577\left(\mathrm{~d}, \mathrm{~J}=9.2 \mathrm{~Hz}, \mathrm{C}^{1} \mathrm{H}\right), 7.026-$ $7.97\left(\mathrm{~m}, 8 \mathrm{H}_{\mathrm{ar}}\right) .{ }^{13} \mathrm{C}: 15.72(\mathrm{C}-5), 20.85\left(\mathrm{ArCH}_{3}\right), 21.30(\mathrm{C}-4), 24.10$ (C-6), 52.57 (C-7), 60.82 (C-1), 64.53 (C-3).

${ }^{1} \mathrm{H}: 1.666-1.821\left(\mathrm{~m}, 1 \mathrm{H}, \mathrm{H}_{\mathrm{B}}\right), 1.876\left(\mathrm{~s}, 3 \mathrm{H}, \mathrm{SCH}_{3}\right), 2.155-2.355$ $\left(\mathrm{m}, 1 \mathrm{H}, \mathrm{H}_{\mathrm{A}}\right), 2.474\left(\mathrm{~s}, 3 \mathrm{H}, \mathrm{ArCH}_{3}\right), 3.466-3.732\left(\mathrm{~m}, 1 \mathrm{H}, \mathrm{H}_{\mathrm{x}}\right), 7.23-$ $7.857\left(\mathrm{~m}, 9 \mathrm{H}_{\mathrm{ar}}\right) .{ }^{13} \mathrm{C}: 15.39\left(\mathrm{SCH}_{3}\right), 20.27\left(\mathrm{CH}_{2}\right), 21.76\left(\mathrm{ArCH}_{3}\right)$, $31.77(\mathrm{CH}), 52.44\left(\mathrm{C}_{\text {quart. }}\right)$.

${ }^{1} \mathbf{H}: 1.554-1.710\left(\mathrm{~m}, 1 \mathrm{H}, \mathrm{H}_{\mathrm{B}}\right), 1.887\left(\mathrm{~s}, 3 \mathrm{H}, \mathrm{SCH}_{3}\right), 2.131-2.253$ $\left(\mathrm{m}, 1 \mathrm{H}, \mathrm{H}_{\mathrm{A}}\right), 2.468\left(\mathrm{~s}, 3 \mathrm{H}, \mathrm{ArCH}_{3}\right), 3.445-3.623\left(\mathrm{~m}, 1 \mathrm{H}, \mathrm{H}_{\mathrm{x}}\right), 3.78$ (s, 3H, $\left.\mathrm{OCH}_{3}\right), 6.85-7.942\left(\mathrm{~m}, 8 \mathrm{H}_{\mathrm{ar}}\right) .{ }^{13} \mathrm{C}: 15.34\left(\mathrm{SCH}_{3}\right), 20.34$ $\left(\mathrm{CH}_{2}\right), 21.64\left(\mathrm{ArCH}_{3}\right), 31.20(\mathrm{CH}), 52.25\left(\mathrm{C}_{\text {quart. }}\right), 55.18\left(\mathrm{OCH}_{3}\right)$.

${ }^{1} \mathbf{H}: 1.565-1.724\left(\mathrm{~m}, 1 \mathrm{H}, \mathrm{H}_{\mathrm{B}}\right), 2.275\left(\mathrm{~s}, 3 \mathrm{H}, \mathrm{CH}_{3} \mathrm{C}_{6} \mathrm{H}_{4} \mathrm{~S}\right), 2.360-$ $2.539\left(\mathrm{~m}, 1 \mathrm{H}, \mathrm{H}_{\mathrm{A}}\right), 2.51\left(\mathrm{~s}, 3 \mathrm{H}, \mathrm{CH}_{3} \mathrm{C}_{6} \mathrm{H}_{4} \mathrm{SO}_{2}\right), 3.482-3.681(\mathrm{~m}, 1 \mathrm{H}$, $\left.\mathrm{H}_{\mathrm{X}}\right), 6.921-7.971\left(\mathrm{~m}, 13 \mathrm{H}_{\mathrm{ar}}\right) .{ }^{13} \mathrm{C}: 19.81\left(\underline{C H}_{3} \mathrm{C}_{6} \mathrm{H}_{4} \mathrm{~S}\right), 20.98$ $\left(\mathrm{CH}_{3} \mathrm{C}_{6} \mathrm{H}_{4} \mathrm{SO}_{2}\right), 21.57\left(\mathrm{CH}_{2}\right), 34.17(\mathrm{CH}), 54.45\left(\mathrm{C}_{\text {quart. }}\right)$.

${ }^{1} \mathrm{H}: 1.532-1.672\left(\mathrm{~m}, 1 \mathrm{H}, \mathrm{H}_{\mathrm{B}}\right), 2.288\left(\mathrm{~s}, 3 \mathrm{H}, \mathrm{CH}_{3} \mathrm{C}_{6} \mathrm{H}_{4} \mathrm{~S}\right), 2.335$ $2.503\left(\mathrm{~m}, 1 \mathrm{H}, \mathrm{H}_{\mathrm{A}}\right), 2.464\left(\mathrm{~s}, 3 \mathrm{H}, \mathrm{CH}_{3} \mathrm{C}_{6} \mathrm{H}_{4} \mathrm{SO}_{2}\right), 3.439-3.635(\mathrm{~m}$, $\left.1 \mathrm{H}, \mathrm{H}_{\mathrm{X}}\right), 3.772\left(\mathrm{~s}, 3 \mathrm{H}, \mathrm{OCH}_{3}\right), 6.83-7.968\left(\mathrm{~m}, 12 \mathrm{H}_{\mathrm{ar}}\right) .{ }^{13} \mathrm{C}: 20.71$ $\left(\mathrm{CH}_{3} \mathrm{C}_{6} \mathrm{H}_{4} \mathrm{~S}\right), 20.98\left(\mathrm{CH}_{3} \mathrm{C}_{6} \mathrm{H}_{4} \mathrm{SO}_{2}\right), 21.57\left(\mathrm{CH}_{2}\right), 33.72(\mathrm{CH}), 54,45$ $\left(\mathrm{C}_{\text {quart. }}\right), 55.17\left(\mathrm{OCH}_{3}\right)$.

${ }^{1} \mathrm{H}: 2.245$ (s, 3H, $\left.\mathrm{SCH}_{3}\right), 2.463$ (s, 3H, $\left.\mathrm{ArCH}_{3}\right), 4.984$ (s, 2H, $2 \mathrm{CH}$ ), $5.413,5.492\left(2 \mathrm{~d}, \mathrm{~J}=-2.1 \mathrm{~Hz}, 2 \mathrm{H}, \mathrm{CH}_{2}\right), 7.369,7.834(2 \mathrm{~d}, \mathrm{~J}=8.2 \mathrm{~Hz}$, $\left.4 \mathrm{H}_{\mathrm{ar}}\right) .{ }^{13} \mathrm{C}: 15.61\left(\mathrm{SCH}_{3}\right), 21.68\left(\mathrm{ArCH}_{3}\right), 58.51(\mathrm{C}-6), 65.44(\mathrm{C}-1$, C-5), 105.40 (C-3), 129.28, 129.57, 145.09 ( $\left.\mathrm{C}_{\text {ar }}\right)$.

p r-1-phenylsulfonyl-t-2,t-3-dimeth- $\quad$ calc. C 49.98 H 5.59 oxy-t-1-methylthiofound C 49.90 H 5.51 $\mathrm{C}_{12} \mathrm{H}_{16} \mathrm{O}_{4} \mathrm{~S}_{2}$ (288.4)

IR $(\mathrm{KBr})\left(\mathrm{cm}^{-1}\right)$ : 1335, 1320, 1300, $1150\left(\mathrm{SO}_{2}\right)$

m.p. $\left(\mathrm{CH}_{3} \mathrm{OH}\right): 80^{\circ} \mathrm{C}$ q r-1-(4-methylphenylsulfonyl)-t-2, t-3-dimethoxy-t-1-methylthio$\mathrm{C}_{13} \mathrm{H}_{18} \mathrm{O}_{4} \mathrm{~S}_{2}$ (302.4)

m.p. $\left(\mathrm{CH}_{3} \mathrm{OH}\right): 137^{\circ} \mathrm{C}$ calc. C 51.63 H 6.00 S 21.21 found C 51.99 H 6.17 S 20.80

$\mathrm{M}^{+}$at $\mathrm{m} / \mathrm{z} 302$ IR $(\mathrm{KBr})\left(\mathrm{cm}^{-1}\right)$ : $1288,1275,1140$, $1135\left(\mathrm{SO}_{2}\right)$
${ }^{1} \mathrm{H}: 2.289\left(\mathrm{~s}, 3 \mathrm{H}, \mathrm{SCH}_{3}\right), 3.395\left(\mathrm{~s}, 6 \mathrm{H}, \mathrm{OCH}_{3}\right), 4.077(\mathrm{~s}, 2 \mathrm{H}, \mathrm{CH})$, $7.579\left(\mathrm{t}, \mathrm{J}=7.2 \mathrm{~Hz}, 2 \mathrm{H}_{\mathrm{ar}}\right), 7.666\left(\mathrm{t}, \mathrm{J}=7.4 \mathrm{~Hz}, 1 \mathrm{H}_{\mathrm{ar}}\right), 7.989(\mathrm{~d}, \mathrm{~J}=$ $\left.7.8 \mathrm{~Hz}, 2 \mathrm{H}_{\mathrm{ar}}\right) .{ }^{13} \mathrm{C}: 15.10\left(\mathrm{SCH}_{3}\right), 52.34(\mathrm{C}-1), 60.04\left(\mathrm{OCH}_{3}\right), 66.12$ (C-2,C-3), 128.71, 129.24, 133.76, $137.54\left(\mathrm{C}_{\mathrm{ar}}\right)$.

${ }^{1} \mathbf{H}: 2.292\left(\mathrm{~s}, 3 \mathrm{H}, \mathrm{SCH}_{3}\right), 2.450\left(\mathrm{~s}, 3 \mathrm{H}, \mathrm{ArCH}_{3}\right), 3.400\left(\mathrm{~s}, 6 \mathrm{H}, \mathrm{OCH}_{3}\right)$, $4.062(\mathrm{~s}, 2 \mathrm{H}, \mathrm{CH}), 7.277-7.913\left(\mathrm{~m}, 4 \mathrm{H}_{\mathrm{ar}}\right){ }^{\mathbf{1 3}} \mathrm{C}: 14.94\left(\mathrm{SCH}_{3}\right), 21.37$ $\left(\mathrm{ArCH}_{3}\right), 52.24(\mathrm{C}-1), 59.85\left(\mathrm{OCH}_{3}\right), 65.89(\mathrm{C}-2, \mathrm{C}-3)$. 


\begin{tabular}{|c|c|c|}
\hline $\begin{array}{l}\underline{\mathbf{r}} \mathrm{r} \text {-1-(4-chlorophenylsulfonyl)-t-2, } \\
\text { t-3-dimethoxy-t-1-methylthio- } \\
\qquad \mathrm{C}_{12} \mathrm{H}_{15} \mathrm{ClO}_{4} \mathrm{~S}_{2} \\
\quad(322.8) \\
\text { m.p. }\left(\mathrm{CH}_{3} \mathrm{OH}\right): 107^{\circ} \mathrm{C}\end{array}$ & $\begin{array}{l}\text { calc. C } 44.65 \mathrm{H} 4.68 \\
\text { found C } 44.52 \mathrm{H} 4.68 \\
\operatorname{IR~}\left(\mathrm{KBr}\left(\mathrm{cm}^{-1}\right):\right. \\
1320,1310,1295 \\
1155\left(\mathrm{SO}_{2}\right) \\
\end{array}$ & $\begin{array}{l}{ }^{1} \mathbf{H}: 2.331\left(\mathrm{~s}, 3 \mathrm{H}, \mathrm{SCH}_{3}\right), 3.407\left(\mathrm{~s}, 6 \mathrm{H}, \mathrm{OCH}_{3}\right), 4.056(\mathrm{~s}, 2 \mathrm{H}, \mathrm{CH}), \\
7.555,7.922\left(\mathrm{~d}, \mathrm{~J}=8.6 \mathrm{~Hz}, 4 \mathrm{H}_{\mathrm{ar}}\right) .{ }^{13} \mathrm{C}: 15.24\left(\mathrm{SCH}_{3}\right), 52.44(\mathrm{C}-1), \\
60.17\left(\mathrm{OCH}_{3}\right), 66.25(\mathrm{C}-2, \mathrm{C}-3), 129.08,130.68,135.98,140.65 \\
\left(\mathrm{C}_{\mathrm{ar}}\right) .\end{array}$ \\
\hline $\begin{array}{l}\underline{\mathbf{s}} \mathrm{r} \text {-1-(methylphenylsulfonyl)-t-2,t-3- } \\
\text { dimethoxy-t-(4-methylphenylthio)- } \\
\qquad \mathrm{C}_{19} \mathrm{H}_{22} \mathrm{O}_{4} \mathrm{~S}_{2} \\
(378.5)\end{array}$ & $\begin{array}{l}\text { calc. C } 60.30 \text { H } 5.82 \\
\text { found C } 59.93 \text { H } 5.84 \\
\qquad \mathrm{M}^{+} \text {at } \mathrm{m} / \mathrm{z} 378\end{array}$ & 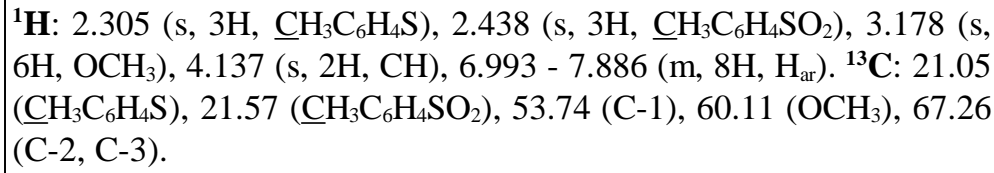 \\
\hline m.p. $\left(\mathrm{CH}_{3} \mathrm{C}\right.$ & & \\
\hline
\end{tabular}

continued

\begin{tabular}{|c|c|c|}
\hline -cyclopropane $\underline{7}$ & & $\mathrm{NMR}\left(\mathrm{CDCl}_{3} / \mathrm{TMS}\right), \delta(\mathrm{ppm})$ \\
\hline $\begin{array}{l}\text { t r-1-(4-methylphenylsulfonyl)-c-2, } \\
\text { t-3-dimethoxy-t-1-methylthio- } \\
\qquad \mathrm{C}_{13} \mathrm{H}_{18} \mathrm{O}_{4} \mathrm{~S}_{2} \\
(302.4) \\
\text { m.p. }\left(\mathrm{CH}_{3} \mathrm{OH}\right): 118^{\circ} \mathrm{C}\end{array}$ & $\begin{array}{l}\text { calc. C } 51.63 \text { H } 6.00 \\
\text { found C } 51.1 \text { H } 5.79 \\
\qquad \mathrm{M}^{+} \text {at } \mathrm{m} / \mathrm{z} 302\end{array}$ & $\begin{array}{l}{ }^{1} \mathbf{H}: 2.298\left(\mathrm{~s}, 3 \mathrm{H}, \mathrm{SCH}_{3}\right), 2.455\left(\mathrm{~s}, 3 \mathrm{H}, \mathrm{ArCH}_{3}\right), 3.240\left(\mathrm{~s}, 3 \mathrm{H}, \mathrm{OCH}_{3}\right), \\
3.586\left(\mathrm{~s}, 3 \mathrm{H}, \mathrm{OCH}_{3}\right), 3.848(\mathrm{~d}, \mathrm{~J}=3.8 \mathrm{~Hz}, 1 \mathrm{H}, \mathrm{CH}), 4.628(\mathrm{~d}, \mathrm{~J}=3.8 \\
\mathrm{Hz}, 1 \mathrm{H}, \mathrm{CH}), 7.260-7.948\left(\mathrm{~m}, 4 \mathrm{H}_{\mathrm{ar}}\right) .\end{array}$ \\
\hline $\begin{array}{l}\text { u् r-1-(4-methylphenylsulfonyl)-c-2, } \\
\text { t-3-dimethoxy-t-1-(4-methylphenyl- } \\
\text { thio)- } \\
\qquad \mathrm{C}_{19} \mathrm{H}_{22} \mathrm{O}_{4} \mathrm{~S}_{2} \\
\quad(378.5) \\
\text { m.p. }\left(\mathrm{CH}_{3} \mathrm{OH}\right): 177^{\circ} \mathrm{C}\end{array}$ & $\mathrm{M}^{+}$at $\mathrm{m} / \mathrm{z} 378$ & $\begin{array}{l}1 \mathbf{H}: 2.334\left(\mathrm{~s}, 3 \mathrm{H}, \underline{\mathrm{CH}}_{3} \mathrm{C}_{6} \mathrm{H}_{4} \mathrm{~S}\right), 2.455\left(\mathrm{~s}, 3 \mathrm{H}, \underline{\mathrm{CH}}_{3} \mathrm{C}_{6} \mathrm{H}_{4} \mathrm{SO}_{2}\right), 3.240(\mathrm{~s}, \\
\left.3 \mathrm{H}, \mathrm{OCH}_{3}\right), 3.276\left(\mathrm{~s}, 3 \mathrm{H}, \mathrm{OCH}_{3}\right), 3.637(\mathrm{~d}, \mathrm{~J}=3.9 \mathrm{~Hz}, 1 \mathrm{H}, \mathrm{CH}), \\
4.690(\mathrm{~d}, \mathrm{~J}=3.9 \mathrm{~Hz}, 1 \mathrm{H}, \mathrm{CH}), 7.055-7.988\left(\mathrm{~m}, 8 \mathrm{H}_{\mathrm{ar}}\right) .\end{array}$ \\
\hline $\begin{array}{l}\text { v spiro-(r-1-sulfonylethylene-t-1- } \\
\text { thio)-t-2,t-3-dimethoxy- } \\
+ \text { spiro-(r-1-sulfonylethylene-t-1- } \\
\text { thio)-c-2, t-3-dimethoxy (mixture of } \\
\text { isomers) } \\
\qquad \mathrm{C}_{7} \mathrm{H}_{12} \mathrm{O}_{4} \mathrm{~S}_{2} \\
(224.3)\end{array}$ & $\begin{array}{l}\text { oil } \\
\text { IR }(\text { neat })\left(\mathrm{cm}^{-1}\right): \\
1340,1330,1320 \\
1170,1150,1135 \\
\left(\mathrm{SO}_{2}\right)\end{array}$ & $\begin{array}{l}{ }^{1} \mathbf{H}: 3.266-3.300\left(\mathrm{~m}, 1 \mathrm{H}, \mathrm{CH}_{2}\right), 3.432-3.540\left(\mathrm{~m}, 3 \mathrm{H}, \mathrm{CH}_{2}\right), 3.577 \\
\left(\mathrm{~s}, 6 \mathrm{H}, \mathrm{OCH}_{3}\right), 3.768(\mathrm{~s}, 2 \mathrm{H}, \mathrm{CH}) .\end{array}$ \\
\hline $\begin{array}{l}\text { w spiro-(r-1-sulfonylpropylene-t-1- } \\
\text { thio)-t-2,t-3-dimethoxy- } \\
\qquad \mathrm{C}_{8} \mathrm{H}_{14} \mathrm{O}_{4} \mathrm{~S}_{2} \\
(238.3) \\
\text { m.p. }\left(\mathrm{CH}_{3} \mathrm{OH}\right): 105^{\circ} \mathrm{C}\end{array}$ & $\begin{array}{l}\mathrm{IR}(\mathrm{KBr})\left(\mathrm{cm}^{-1}\right): \\
1310,1295,1140 \\
1115\left(\mathrm{SO}_{2}\right)\end{array}$ & $\begin{array}{l}{ }^{1} \mathbf{H}: 2.609-2.680\left(\mathrm{~m}, 2 \mathrm{H}, \mathrm{CH}_{2}\right), 2.760-2.787\left(\mathrm{~m}, 2 \mathrm{H}, \mathrm{CH}_{2}\right), 3.110- \\
3.140\left(\mathrm{~m}, 2 \mathrm{H}, \mathrm{CH}_{2}\right), 3.576\left(\mathrm{~s}, 6 \mathrm{H}, \mathrm{OCH}_{3}\right), 3.976(\mathrm{~s}, 2 \mathrm{H}, \mathrm{CH}) .{ }^{13} \mathrm{C}: \\
28.20\left(\mathrm{C}-\mathrm{CH}_{2}-\mathrm{C}\right), 28.81\left(\mathrm{CH}_{2} \mathrm{~S}\right), 47.10\left(\mathrm{CH}_{2} \mathrm{SO}_{2}\right), 52.04(\mathrm{C}-1), 60.09 \\
\left(\mathrm{OCH}_{3}\right), 63.42(\mathrm{C}-2, \mathrm{C}-3) .\end{array}$ \\
\hline $\begin{array}{c}\underline{\mathbf{x}} \text { spiro-(r-1-sulfonyl-o-phenylene-t- } \\
\text { 1-thio)-t-2,t-3-dimethoxy- } \\
\quad \mathrm{C}_{11} \mathrm{H}_{12} \mathrm{O}_{4} \mathrm{~S}_{2} \\
(272.3) \\
\text { m.p. }\left(\mathrm{CH}_{3} \mathrm{OH}\right): 139^{\circ} \mathrm{C}\end{array}$ & $\begin{array}{l}\text { calc. C } 48.51 \mathrm{H} 4.44 \\
\text { found } \mathrm{C} 48.40 \mathrm{H} 4.40 \\
\mathrm{M}^{+} \text {at } \mathrm{m} / \mathrm{z} 272 \\
\mathrm{IR}(\mathrm{KBr})\left(\mathrm{cm}^{-1}\right) \text { : } \\
1300,1165,1140 \\
\left(\mathrm{SO}_{2}\right)\end{array}$ & $\begin{array}{l}{ }^{1} \mathbf{H}: 3.553\left(\mathrm{~s}, 6 \mathrm{H}, \mathrm{OCH}_{3}\right), 4.034(\mathrm{~s}, 2 \mathrm{H}, \mathrm{CH}), 7.373-7.420\left(\mathrm{~m}, 2 \mathrm{H}_{\mathrm{ar}}\right), \\
7.521-7.564\left(\mathrm{~m}, 1 \mathrm{H}_{\mathrm{ar}}\right), 7.707-7.727\left(\mathrm{~m}, 1 \mathrm{H}_{\mathrm{ar}}\right) .{ }^{13} \mathrm{C}: 50.67(\mathrm{C}-1), \\
59.74\left(\mathrm{OCH}_{3}\right), 62.44(\mathrm{C}-2, \mathrm{C}-3), 123.10,125.42,133.56,134.42 \\
\left(\mathrm{C}_{\mathrm{ar}}\right) .\end{array}$ \\
\hline
\end{tabular}

Trithioorthoformate S,S, $S^{\prime}, S^{\prime}$-tetroxides 28: During the aforementioned cyclopropanations depending on the relative reactivities of generated carbenes $\underline{\mathbf{3}}$ and of applied alkenes $\underline{\mathbf{6}}$ larger or minor amounts of $\beta$-disulfones $\underline{\mathbf{2 8}}$ (7-12\% yield, calculation basis $1 \mathrm{~mol} \mathbf{2 8}$ from $2 \mathrm{~mol} \underline{4}$ according to Scheme 9) were isolated as highly crystalline compounds. Without addition of a trapping olefin $\beta$-disulfone $\mathbf{2 8 a}$ could be obtained from $\alpha$-chloro- $\alpha$-sulfonyl thioether 4b in 57.7\% yield: Bis(4-methylphenylsulfonyl) methylthio-methane (28a): m.p. $139{ }^{\circ} \mathrm{C}$ (methanol), $\mathrm{C}_{16} \mathrm{H}_{18} \mathrm{O}_{4} \mathrm{~S}_{3}$ (370.5) calc. C $51.87 \mathrm{H} 4.90$ found C 52.31 H 4.83; IR (KBr): 1326, 1318, $1148,1140\left(\mathrm{SO}_{2}\right) \mathrm{cm}^{-1}$; ${ }^{1} \mathrm{H}-\mathrm{NMR}\left(\mathrm{CDCl}_{3} / \mathrm{TMS}\right): \delta=2.318\left(\mathrm{~s}, 3 \mathrm{H}, \mathrm{SCH}_{3}\right), 2.471\left(\mathrm{~s}, 6 \mathrm{H}, \mathrm{ArCH}_{3}\right), 4.880(\mathrm{~s}, 1 \mathrm{H}, \mathrm{CH}), 7.260$ - $7.961(\mathrm{~m}$, $8 \mathrm{H}_{\text {ar }}$ ) ppm. Bis(4-chlorophenylsulfonyl) methylthio-methane (28b): m.p. 195-196 ${ }^{\circ} \mathrm{C}$ (methanol), $\mathrm{C}_{14} \mathrm{H}_{12} \mathrm{Cl}_{2} \mathrm{O}_{4} \mathrm{~S}_{3}$ (411.4) calc. C $40.88 \mathrm{H} 2.94$ found C $41.20 \mathrm{H} \mathrm{2.91;} \mathrm{IR} \mathrm{(KBr):} \mathrm{1330,} \mathrm{1325,} 1150\left(\mathrm{SO}_{2}\right) \mathrm{cm}^{-1}$; ${ }^{1} \mathrm{H}-\mathrm{NMR}$ $\left(\mathrm{d}_{6} \text {-DMSO/TMS }\right)^{48}: \delta=2.27\left(\mathrm{~s}, 3 \mathrm{H}, \mathrm{SCH}_{3}\right), 6.93(\mathrm{~s}, 1 \mathrm{H}, \mathrm{CH}), 7.68-8.15\left(\mathrm{~m}, 8 \mathrm{H}_{\mathrm{ar}}\right)$ ppm. Bis $(4-$ methylphenylsulfonyl)-(4-methylphenylthio)-methane (28c): m.p. $158{ }^{\circ} \mathrm{C}$ (methanol), $\mathrm{C}_{22} \mathrm{H}_{22} \mathrm{~S}_{3} \mathrm{O}_{4}(446.6)$, $\mathrm{M}^{+}$at $\mathrm{m} / \mathrm{z}$ 446; ${ }^{1} \mathrm{H}-\mathrm{NMR}\left(\mathrm{CDCl}_{3} / \mathrm{TMS}\right): \delta=2.297\left(\mathrm{~s}, 3 \mathrm{H}, \mathrm{CH}_{3} \mathrm{C}_{6} \mathrm{H}_{4} \mathrm{~S}\right), 2.481\left(\mathrm{~s}, 6 \mathrm{H}, \mathrm{CH}_{3} \mathrm{C}_{6} \mathrm{H}_{4} \mathrm{SO}_{2}\right), 5.037(\mathrm{~s}, 1 \mathrm{H}$, $\mathrm{CH}), 7.019$ - $7.966\left(\mathrm{~m}, 12 \mathrm{H}_{\mathrm{ar}}\right) \mathrm{ppm}$. 
$\underline{\text { Preparation of organylsulfonyl-organylthio-trimethylsilyl methanes }} \underline{\mathbf{3 3}}$ according to a known procedure described for $\mathbf{3 3 b}^{31}: 41 \mathrm{ml}(0.106 \mathrm{~mol}) 2.5$ molar $\mathrm{n}-\mathrm{BuLi}$ in hexane were added dropwise under nitrogen and stirring to a solution of $0.1 \mathrm{~mol}$ sulfonylthioether $\underline{\mathbf{2 a}-\mathbf{c}}$ in $300 \mathrm{ml}$ absol. THF at $-78^{\circ} \mathrm{C}$. Stirring was continued for $90 \mathrm{~min}, 16$ $\mathrm{ml}(0.126 \mathrm{~mol})$ trimethylsilyl chloride were added at once, and the cooling bath was removed after 5 min. After 2 $\mathrm{h}$ stirring at room temperature the reaction mixture was poured onto 11 ice water and extracted with three 250 $\mathrm{ml}$ portions of dichloromethane. After washing with water $(250 \mathrm{ml})$ and drying over $\mathrm{MgSO}_{4}$ the solvent was evaporated at $30{ }^{\circ} \mathrm{C}$. The oily residue crystallized spontaneously after addition of a few ml of dry ether. Phenyl-[trimethylsilyl-methylthiomethyl] sulfone (33a): $86.5 \%$, colorless crystals, m.p. $84.5{ }^{\circ} \mathrm{C} ; \mathrm{C}_{11} \mathrm{H}_{18} \mathrm{O}_{2} \mathrm{SiS}_{2}$ (274.5), calc. C $48.14 \mathrm{H} 6.61$ found $\mathrm{C} 48.20 \mathrm{H}$ 6.44; IR (KBr): 1300, $1145\left(\mathrm{SO}_{2}\right), 850\left(\mathrm{SiMe}_{3}\right) \mathrm{cm}^{-1}$; ${ }^{1} \mathrm{H}-\mathrm{NMR}$ $\left(\mathrm{CDCl}_{3} / \mathrm{TMS}\right): \delta=0.327\left(\mathrm{t}, \mathrm{J}=3.3 \mathrm{~Hz}, 9 \mathrm{H}, \mathrm{Si}\left(\mathrm{CH}_{3}\right)_{3}\right), 1.675\left(\mathrm{~s}, 3 \mathrm{H}, \mathrm{SCH}_{3}\right), 3.197(\mathrm{~s}, 1 \mathrm{H}, \mathrm{CH}), 7.527-7.565$ $\left(\mathrm{m}, 2 \mathrm{H}_{\mathrm{ar}}\right), 7.623\left(\mathrm{~d}, \mathrm{~J}=7.5 \mathrm{~Hz}, 1 \mathrm{H}_{\mathrm{ar}}\right), 8.003\left(\mathrm{~d}, \mathrm{~J}=7 \mathrm{~Hz}, 2 \mathrm{H}_{\mathrm{ar}}\right)$ ppm. ${ }^{13} \mathrm{C}-\mathrm{NMR}\left(\mathrm{CDCl}_{3} / \mathrm{TMS}\right): \delta=-1.22$ $\left(\mathrm{Si}\left(\mathrm{CH}_{3}\right)_{3}\right), 18.24\left(\mathrm{SCH}_{3}\right), 61.43(\mathrm{CH}), 128.63,129.15,133.29,139.72\left(\mathrm{C}_{\mathrm{ar}}\right)$ ppm. 4-Chlorophenyl[trimethylsilyl-methylthiomethyl] sulfone $(\underline{\mathbf{3 3 c}}): \sim 100 \%$, colorless crystals, m.p. $112{ }^{\circ} \mathrm{C} ; \mathrm{C}_{11} \mathrm{H}_{17} \mathrm{ClO}_{2} \mathrm{SiS}_{2}(308.1)$; IR (KBr): 1305, $1140\left(\mathrm{SO}_{2}\right), 840\left(\mathrm{SiMe}_{3}\right) \mathrm{cm}^{-1} ;{ }_{1}^{1} \mathrm{H}-\mathrm{NMR}\left(\mathrm{CDCl}_{3} / \mathrm{TMS}\right): \delta=0.333\left(\mathrm{t}, \mathrm{J}=3.2 \mathrm{~Hz}, 9 \mathrm{H}, \mathrm{Si}\left(\mathrm{CH}_{3}\right)_{3}\right)$, $1.749\left(\mathrm{~s}, 3 \mathrm{H}, \mathrm{SCH}_{3}\right), 3.177(\mathrm{~s}, 1 \mathrm{H}, \mathrm{CH}), 7.528\left(\mathrm{~d}, \mathrm{~J}=8.5 \mathrm{~Hz}, 2 \mathrm{H}_{\mathrm{ar}}\right), 7.945(\mathrm{~d}, \mathrm{~J}=8.7 \mathrm{~Hz}, 2 \mathrm{H}$ ar $) \mathrm{ppm}$; ${ }^{13} \mathrm{C}-\mathrm{NMR}\left(\mathrm{CDCl}_{3} / \mathrm{TMS}\right): \delta=-1.14\left(\mathrm{Si}\left(\mathrm{CH}_{3}\right)_{3}\right), 18.24\left(\mathrm{SCH}_{3}\right), 61.52(\mathrm{CH}), 128.97,130.68,138.28,140.09\left(\mathrm{C}_{\mathrm{ar}}\right)$

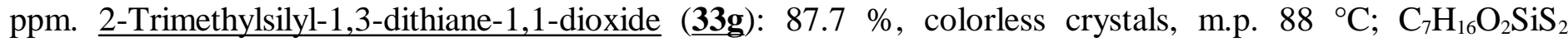
(224.4), $\mathrm{M}^{+}$at $\mathrm{m} / \mathrm{z}$ 224; IR (KBr): 1320, 1300, 1280, $1140\left(\mathrm{SO}_{2}\right) \mathrm{cm}^{-1} ;{ }^{1} \mathrm{H}-\mathrm{NMR}\left(\mathrm{CDCl}_{3} / \mathrm{TMS}\right): \delta=0.333(\mathrm{~s}, 9$ $\left.\mathrm{H}, \mathrm{Si}\left(\mathrm{CH}_{3}\right)_{3}\right), 2.450-2.518\left(\mathrm{~m}, 1 \mathrm{H}, \mathrm{CH}_{2}\right), 2.599-2.670\left(\mathrm{~m}, 1 \mathrm{H}, \mathrm{CH}_{2}\right), 2.683-2.774\left(\mathrm{~m}, 1 \mathrm{H}, \mathrm{CH}_{2}\right), 2.966-$ $3.078\left(\mathrm{~m}, 1 \mathrm{H}, \mathrm{CH}_{2}\right), 3.106-3.199\left(\mathrm{~m}, 1 \mathrm{H}, \mathrm{CH}_{2}\right), 3.690$ (s, $\left.1 \mathrm{H}, \mathrm{CH}\right)$ ppm.; ${ }^{13} \mathrm{C}-\mathrm{NMR}\left(\mathrm{CDCl}_{3} / \mathrm{TMS}\right): \delta=-1.40$ $\left(\mathrm{Si}\left(\mathrm{CH}_{3}\right)_{3}\right), 28.87\left(\mathrm{C}^{\left.-\mathrm{CH}_{2}-\mathrm{C}\right),} 28.99\left(\mathrm{CH}_{2} \mathrm{~S}\right), 53.72\left(\mathrm{CH}_{2} \mathrm{SO}_{2}\right), 54.52\left(\mathrm{~S}-\mathrm{CH}-\mathrm{SO}_{2}\right)\right.$ ppm. 2-Trimethylsilyl-

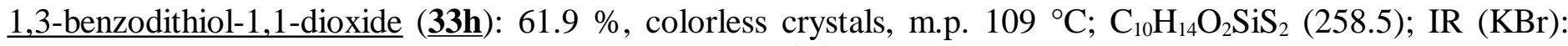
$1580\left(\mathrm{C}=\mathrm{C}_{\mathrm{ar}}\right), 1315,1180,1155\left(\mathrm{SO}_{2}\right), 850\left(\mathrm{SiMe}_{3}\right) \mathrm{cm}^{-1} ;{ }^{1} \mathrm{H}-\mathrm{NMR}\left(\mathrm{CDCl}_{3} / \mathrm{TMS}\right): \delta=0.381\left(\mathrm{~s}, 9 \mathrm{H}, \mathrm{Si}\left(\mathrm{CH}_{3}\right)_{3}\right)$, $4.023(\mathrm{~s}, 1 \mathrm{H}, \mathrm{CH}), 7.301-7.345\left(\mathrm{~m}, 2 \mathrm{H}_{\mathrm{ar}}\right), 7.470-7.511\left(\mathrm{~m}, 1 \mathrm{H}_{\mathrm{ar}}\right), 7.674\left(\mathrm{~d}, \mathrm{~J}=7.9 \mathrm{~Hz}, 1 \mathrm{H}_{\mathrm{ar}}\right) \mathrm{ppm}$; ${ }^{13} \mathrm{C}-\mathrm{NMR}\left(\mathrm{CDCl}_{3} / \mathrm{TMS}\right): \delta=-2.22\left(\mathrm{Si}\left(\mathrm{CH}_{3}\right)_{3}\right), 52.76(\mathrm{CH}), 122.87,125.13,126.31,133.18,135.70,136.48$ $\left(\mathrm{C}_{\mathrm{ar}}\right) \mathrm{ppm}$.

Generation of carbene dimers 35 . Method I: $2.6 \mathrm{ml}(6.5 \mathrm{mmol})$ 2.5-molar n-BuLi in hexane were added dropwise under nitrogen to a solution of $6.4 \mathrm{mmol}$ silylsulfone $\underline{\mathbf{3 3 a}-\mathbf{c}}$ in $50 \mathrm{ml}$ absol. THF and the mixture was stirred for $90 \mathrm{~min}, 0.83 \mathrm{~g}(7.6 \mathrm{mmol})$ tert. butylhypochlorite was added at once; after removal of the cooling bath the mixture was stirred for $16 \mathrm{~h}$ at room temperature. Work-up as mentioned in the preceding section yielded the corresponding carbene dimer as the Z-isomer. Z-1,2-Bis(phenylsulfonyl)-1,2-bis(methylthio)-ethylene ( $35.7 \%$, yellowish crystals, m.p. $205{ }^{\circ} \mathrm{C}$ (glacial acetic acid), $\mathrm{C}_{16} \mathrm{H}_{16} \mathrm{O}_{4} \mathrm{~S}_{4}$ (400.6) calc. $\mathrm{C} 47.98 \mathrm{H} 4.03$ found $\mathrm{C}$ $48.0 \mathrm{H}$ 4.01, $\mathrm{M}^{+}$at $\mathrm{m} / \mathrm{z} 400 ; \mathrm{IR}(\mathrm{KBr})$ : no olefinic $\mathrm{C}=\mathrm{C}$, only $1585\left(\mathrm{C}=\mathrm{C}_{\mathrm{ar}}\right), 1325,1150\left(\mathrm{SO}_{2}\right) \mathrm{cm}^{-1} ; \mathrm{UV}$ $\left(\mathrm{CH}_{2} \mathrm{Cl}_{2}\right): \lambda(\log \varepsilon)=241(4.05), 357(3.94) \mathrm{nm} ;{ }^{1} \mathrm{H}-\mathrm{NMR}\left(\mathrm{CDCl}_{3} / \mathrm{TMS}\right): \delta=2.235\left(\mathrm{~s}, 6 \mathrm{H}, \mathrm{SCH}_{3}\right), 7.583(\mathrm{t}, \mathrm{J}$ $\left.=7.2 \mathrm{~Hz}, 4 \mathrm{H}_{\mathrm{ar}}\right), 7.670\left(\mathrm{t}, \mathrm{J}=7.5 \mathrm{~Hz}, 2 \mathrm{H}_{\mathrm{ar}}\right), 8.333\left(\mathrm{~d}, \mathrm{~J}=7.5 \mathrm{~Hz}, 4 \mathrm{H}_{\mathrm{ar}}\right)$ ppm. ${ }^{13} \mathrm{C}-\mathrm{NMR}\left(\mathrm{CDCl}_{3} / \mathrm{TMS}\right): \delta=$ $16.85\left(\mathrm{SCH}_{3}\right), 128.67,130.00,133.90,139.39,151.34\left(\mathrm{C}_{\mathrm{ar}}\right) \mathrm{ppm}$. Z-1,2-Bis(4-methylphenylsulfonyl)-1,2-bis (methylthio)-ethylene (Z-35b): $3.6 \%$, yellow crystals, m.p. $198{ }^{\circ} \mathrm{C}$ (glacial acetic acid), $\mathrm{C}_{18} \mathrm{H}_{20} \mathrm{O}_{4} \mathrm{~S}_{4}$ (428.6) calc. C $50.44 \mathrm{H} 4.70$ found $\mathrm{C} 50.50 \mathrm{H} \mathrm{4.71;} \mathrm{M}^{+}$at m/z 428; IR $(\mathrm{KBr})$ : no olefinic $\mathrm{C}=\mathrm{C}$, only $1595\left(\mathrm{C}=\mathrm{C}_{\mathrm{ar}}\right), 1310$, $1150\left(\mathrm{SO}_{2}\right) \mathrm{cm}^{-1} ; \mathrm{UV}\left(\mathrm{CH}_{2} \mathrm{Cl}_{2}\right): \lambda(\log \varepsilon)=265(3.96), 352(3.96) \mathrm{nm} ;{ }^{1} \mathrm{H}-\mathrm{NMR}\left(\mathrm{CDCl}_{3} / \mathrm{TMS}\right): \delta=2.221(\mathrm{~s}, 6$ $\left.\mathrm{H}, \mathrm{SCH}_{3}\right), 2.463\left(\mathrm{~s}, 6 \mathrm{H}, \mathrm{ArCH}_{3}\right), 7.365\left(\mathrm{~d}, \mathrm{~J}=8.4 \mathrm{~Hz}, 4 \mathrm{H}_{\mathrm{ar}}\right), 8.203\left(\mathrm{~d}, \mathrm{~J}=8.4 \mathrm{~Hz}, 4 \mathrm{H}_{\mathrm{ar}}\right) \mathrm{ppm} ;{ }^{13} \mathrm{C}-\mathrm{NMR}$ $\left(\mathrm{CDCl}_{3} / \mathrm{TMS}\right): \delta=16.91\left(\mathrm{SCH}_{3}\right), 21.71\left(\mathrm{ArCH}_{3}\right), 129.32,130.14,136.47,144.99,151.38\left(\mathrm{C}_{\mathrm{ar}}\right) \mathrm{ppm}$. Z-1.2-Bis(4-chlorophenylsulfonyl)-1.2-bis(methylthio)-ethylene (Z-35c): $23.3 \%$, yellow crystals, m.p. $200.5{ }^{\circ} \mathrm{C}$

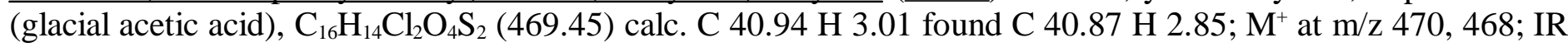
(KBr): no olefinic $\mathrm{C}=\mathrm{C}$, only $1575\left(\mathrm{C}=\mathrm{C}_{\mathrm{ar}}\right), 1315,1155\left(\mathrm{SO}_{2}\right) \mathrm{cm}^{-1} ; \mathrm{UV}\left(\mathrm{CH}_{2} \mathrm{Cl}_{2}\right): \lambda(\log \varepsilon)=231$ (4.16), 264 (4.08), $357(3.95) \mathrm{nm} ;{ }^{1} \mathrm{H}-\mathrm{NMR}\left(\mathrm{CDCl}_{3} / \mathrm{TMS}\right): \delta=2.222\left(\mathrm{~s}, 6 \mathrm{H}, \mathrm{SCH}_{3}\right), 7.549(\mathrm{~d}, \mathrm{~J}=8.7 \mathrm{~Hz}, 4 \mathrm{H}$ ar $), 8.260$ (d, $\left.\mathrm{J}=8.8 \mathrm{~Hz}, 4 \mathrm{H}_{\mathrm{ar}}\right) \mathrm{ppm} ;{ }^{13} \mathrm{C}-\mathrm{NMR}\left(\mathrm{CDCl}_{3} / \mathrm{TMS}\right): \delta=16.60\left(\mathrm{SCH}_{3}\right), 129.09,131.56,137.50,140.88,151.07$ $\left(\mathrm{C}_{\mathrm{ar}}\right) \mathrm{ppm}$. Application of hexachloroethane or tetrachloromethane instead of tert. butylhypochlorite afforded the same compounds in minor yields.

Method II: $7.4 \mathrm{ml}$ (18.5 mmol) 2.5 molar $\mathrm{n}-\mathrm{BuLi}$ in hexane were added dropwise under nitrogen to a solution of $18.4 \mathrm{mmol}$ chlorosulfone $\underline{4 a-c}$ in $60 \mathrm{ml}$ absol. THF at $-78{ }^{\circ} \mathrm{C}$. The solution was stirred for $90 \mathrm{~min}, 2.5 \mathrm{ml}(20$ $\mathrm{mmol}$ ) chlorotrimethylsilane were added and after removal of the cooling bath stirring was continued for $2 \mathrm{~h}$ at room temperature. Further work-up was carried out as described before. Obtained yields of Z-dimers $\underline{\mathbf{3 5}}$ were rather poor: Z-35a $1.6 \%$, Z-35b $5.8 \%$, Z-35c $17.4 \%$.

Use of dichlorosulfones 12a-c under corresponding conditions afforded the following yields: Z-35a $2.5 \%$, Z-35b $3.4 \%$, Z-35c $5.6 \%$. Similar results were obtained under corresponding conditions starting from bromo- 
chlorosulfones 13a-c: Z-35a 7.5\%, Z-35b $4.2 \%$, Z-35c 10.2\%. Z-1.2-Bis(4-methylphenylsulfonyl)-1.2-bis (4-methylphenylthio)-ethylene (Z-35e): $2 \%$ from 4e, $11.2 \%$ from $\underline{\mathbf{1 2 e}}$, yellow crystals, m.p. $208{ }^{\circ} \mathrm{C}$ (methanol), $\mathrm{C}_{30} \mathrm{H}_{28} \mathrm{O}_{4} \mathrm{~S}_{4}(580.8)$ calc. $\mathrm{C} 62.04 \mathrm{H} 4.86$ found C $62.48 \mathrm{H}$ 5.02; IR (KBr): $1595\left(\mathrm{C}=\mathrm{C}_{\mathrm{ar}}\right), 1335,1160\left(\mathrm{SO}_{2}\right) \mathrm{cm}^{-1}$; ${ }^{1} \mathrm{H}-\mathrm{NMR}\left(\mathrm{CDCl}_{3} / \mathrm{TMS}\right): \delta=2.327\left(\mathrm{~s}, 6 \mathrm{H}, \underline{\mathrm{CH}}_{3} \mathrm{C}_{6} \mathrm{H}_{4} \mathrm{~S}\right), 2.449\left(\mathrm{~s}, 6 \mathrm{H}, \mathrm{CH}_{3} \mathrm{C}_{6} \mathrm{H}_{4} \mathrm{SO}_{2}\right), 6.835(\mathrm{~d}, \mathrm{~J}=8.2 \mathrm{~Hz}, 4$ $\left.\mathrm{H}_{\mathrm{ar}}\right), 7.092\left(\mathrm{~d}, \mathrm{~J}=8.1 \mathrm{~Hz}, 4 \mathrm{H}_{\mathrm{ar}}\right), 7.343\left(\mathrm{~d}, \mathrm{~J}=8.1 \mathrm{~Hz}, 4 \mathrm{H}_{\mathrm{ar}}\right), 8.195\left(\mathrm{~d}, \mathrm{~J}=8.4 \mathrm{~Hz}, 4 \mathrm{H}_{\mathrm{ar}}\right)$ ppm; ${ }^{13} \mathrm{C}-\mathrm{NMR}$

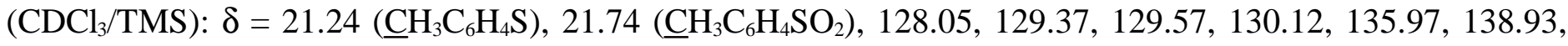
$145.11,151.06\left(\mathrm{C}_{\text {olefin }}+\mathrm{C}_{\mathrm{ar}}\right) \mathrm{ppm}$.

Method III: Solutions of $20 \mathrm{mmol}$ sulfonylthioether $\underline{\mathbf{2 c}}$, respectively $\underline{\mathbf{4 c}}$, in $60 \mathrm{ml}$ absol. THF were lithiated with $8.8 \mathrm{ml}(22 \mathrm{mmol}) 2.5$ molar $\mathrm{n}-\mathrm{BuLi}$ in hexane at $-78^{\circ} \mathrm{C}$ as described before. Oxidation was carried out by dropwise addition of a solution of $2.54 \mathrm{~g}(20 \mathrm{mmol})$ iodine resp. $2.7 \mathrm{~g}(20 \mathrm{mmol})$ phenyl glyoxal in $20 \mathrm{ml}$ absol. THF and stirring for $3 \mathrm{~h}$ at $-78{ }^{\circ} \mathrm{C}$, finally $12 \mathrm{~h}$ at room temperature. Similar work-up as described before afforded Z-35c in $15 \%$ from $\underline{\mathbf{2 c}}$ resp. in $7.5 \%$ from $\underline{\mathbf{4 c}}$ via iodine oxidation, whereas phenyl glyoxal oxidation of $\underline{\mathbf{4 c}}$ afforded Z-35c in $17 \%$ yield, properties as described before.

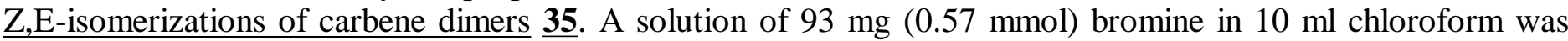
dropped to a stirred solution of $0.5 \mathrm{mmol}$ dimer $\underline{\mathbf{Z}-35 \mathrm{a}-\mathrm{c}}$ in $40 \mathrm{ml}$ chloroform at $0{ }^{\circ} \mathrm{C}$ and stirring was continued for $6 \mathrm{~h}$ at $0{ }^{\circ} \mathrm{C}$ and $12 \mathrm{~h}$ at room temperature. The solution was washed with $50 \mathrm{ml}$ water, the separated water phase was extracted twice with $30 \mathrm{ml}$ chloroform, the combined chloroform phases were dried over $\mathrm{MgSO}_{4}$ and the solvent was removed by destillation under reduced pressure. The oily residue was caused to crystallize after addition of a few ml methanol; $92.5 \% \underline{\mathbf{3 5 a}}$ as $\mathrm{E} / \mathrm{Z}=12.9: 1$-mixture, $64.4 \% \underline{\mathbf{3 5 b}}$ as $\mathrm{E} / \mathrm{Z}=23: 1$-mixture, $90 \%$ $\underline{35 c}$ as $E / Z=10.6 / 1$-mixture, melting points of E-isomers after recrystallization from chloroform have been found to be higher than those of corresponding Z-isomers as had been found in other cases too ${ }^{58}$. E-35a: m.p. $210{ }^{\circ} \mathrm{C}, \mathrm{UV}\left(\mathrm{CH}_{2} \mathrm{Cl}_{2}\right): \lambda(\log \varepsilon)=230$ (3.96), 366 (3.38) nm; ${ }^{1} \mathrm{H}-\mathrm{NMR}\left(\mathrm{CDCl}_{3} / \mathrm{TMS}\right): \delta=2.213\left(\mathrm{~s}, 6 \mathrm{H}, \mathrm{SCH}_{3}\right)$, $7.551\left(\mathrm{t}, \mathrm{J}=7.7 \mathrm{~Hz}, 4 \mathrm{H}_{\mathrm{ar}}\right), 7.659\left(\mathrm{t}, \mathrm{J}=7.3 \mathrm{~Hz}, 4 \mathrm{H}_{\mathrm{ar}}\right), 7.985\left(\mathrm{~d}, \mathrm{~J}=7.6 \mathrm{~Hz}, 4 \mathrm{H}_{\mathrm{ar}}\right) \mathrm{ppm} ;{ }^{13} \mathrm{C}-\mathrm{NMR}$ $\left(\mathrm{CDCl}_{3} / \mathrm{TMS}\right): \delta=20.44\left(\mathrm{SCH}_{3}\right), 128.74,128.89,133.85,140.08,156.96\left(\mathrm{C}_{\mathrm{ar}}\right)$ ppm. E-35b: m.p. $206{ }^{\circ} \mathrm{C}$; UV $\left(\mathrm{CH}_{2} \mathrm{Cl}_{2}\right): \lambda(\log \varepsilon)=231(4.35), 365(3.59) \mathrm{nm} ;{ }^{1} \mathrm{H}-\mathrm{NMR}\left(\mathrm{CDCl}_{3} / \mathrm{TMS}\right): \delta=2.233\left(\mathrm{~s}, 6 \mathrm{H}, \mathrm{SCH}_{3}\right), 2.455(\mathrm{~s}, 6$ $\left.\mathrm{H}, \mathrm{ArCH}_{3}\right), 7.333\left(\mathrm{~d}, \mathrm{~J}=8.1 \mathrm{~Hz}, 4 \mathrm{H}_{\mathrm{ar}}\right), 7.864\left(\mathrm{~d}, \mathrm{~J}=8.3 \mathrm{~Hz}, 4 \mathrm{H}_{\mathrm{ar}}\right)$ ppm; ${ }^{13} \mathrm{C}-\mathrm{NMR}\left(\mathrm{CDCl}_{3} / \mathrm{TMS}\right): \delta=20.59$ $\left(\mathrm{SCH}_{3}\right), 21.69\left(\mathrm{ArCH}_{3}\right), 129.08,129.34,136.97,145.06,156.91\left(\mathrm{C}_{\mathrm{ar}}\right)$ ppm. E-35c: m.p. $234{ }^{\circ} \mathrm{C} ; \mathrm{M}^{+}$at $\mathrm{m} / \mathrm{z} 468$, 470, 472; ${ }^{1} \mathrm{H}-\mathrm{NMR}\left(\mathrm{CDCl}_{3} / \mathrm{TMS}\right): \delta=2.335\left(\mathrm{~s}, 6 \mathrm{H}, \mathrm{SCH}_{3}\right), 7.523\left(\mathrm{~d}, \mathrm{~J}=8.6 \mathrm{~Hz}, 4 \mathrm{H}_{\mathrm{ar}}\right), 7.930(\mathrm{~d}, \mathrm{~J}=8.6 \mathrm{~Hz}, 4$ $\left.\mathrm{H}_{\mathrm{ar}}\right) \mathrm{ppm} ;{ }^{13} \mathrm{C}-\mathrm{NMR}\left(\mathrm{CDCl}_{3} / \mathrm{TMS}\right): \delta=20.98\left(\mathrm{SCH}_{3}\right), 129.11,130.42,138.22,140.88,156.79\left(\mathrm{C}_{\mathrm{ar}}\right)$ ppm. Only E-35c could be obtained completely free of its Z-isomer: At room temperature however, all E-isomers isomerized to their Z-forms either as crystals or as solutions.

Acknowledgement: Financial Support of these investigations by the Deutsche Forschungsgemeinschaft and the Egyptian Government is gratefully acknowledged. We are indebted to Thomas Heisel for drawings in this manuscript, to Professor Kevin Bell from the University of Newcastle (Australia) for helpful comments as well as to Professor Regitz and Mrs. Allester from University of Kaiserslautern for a great number of microanalyses.

\section{Dedicated to Professor U. Schöllkopf, the pioneer of thiocarbene chemistry.}

\section{REFERENCES AND NOTES}

1. Schank, K.; Abdel Wahab, A.A.; Eigen, P.; Jager, J. Tetrahedron 1989, 45, 6667; Presented in part at the $9^{\text {th }}$ International Symposium on the Organic Chemistry of Sulfur (ISOCS) in Riga (UdSSR) 1980.

2. For a comprehensive survey on thiosubstituted carbenes cf.: Schank, K. in Methoden der Organischen Chemie (Houben-Weyl), $4^{\text {th }}$ Edit., Vol. E19b, Carbene (Carbenoide) (Ed. M. Regitz), p. 1682-1745, G. Thieme, Stuttgart 1989.

3. Schöllkopf, U.; Lehmann, G.J.; Paust, J.; Härtel, H.-D. Chem. Ber. 1964, 97, 1527.

4. Schöllkopf, U.; Paust, J. Chem. Ber. 1965, 98, 2221; Schöllkopf, U.; Woerner, F.P.; Wiskott, E. ibid. 1966, 99, 806.

5. Schöllkopf, U.; Wiskott, E. Liebigs Ann. Chem. 1966, 694, 44.

6. cf. $\alpha$ sulfonyl ethers, 17: Schank, K.; Schröder, F.; Weber, A. Liebigs Ann. Chem. 1979, 547.

7. cf. Schank, K. Phosphorus, Sulfur, and Silicon 1991, 58, 207 on p. 218; ref. 2, p. 1731, 1735-1738.

8. Moss, R.A. Acc. Chem. Res. 1980, 13, 58.

9. Abdel Wahab, A.A. unpublished results, Saarbrücken 1984.

10. Regitz, M. Chem. Ber. 1964, 97, 2742. 
11. Hadjiarapoglou, L.; Spyroudis, S.; Varvoglis, A. J. Am. Chem. Soc. 1985, 107, 7178 on p. 7179.

12. a) Jost, K. Ph. D. Dissertation, Saarbrücken 1988; b) Vilsmaier, E.; Sprügel, W. Liebigs Ann. Chem. 1971, $747,151$.

13. For a summary of literature known methods cf. ref. 12a).

14. a) Gibson, D.T. J. Chem. Soc. 1931, 2637; b) Gibson, D.T. J. Chem. Soc. 1932, 1819; c)

Gibson, D.T. J. Chem. Soc. 1937, 1509; cf. Freeman, F. Chem. Rev. 1984, 84, 117.

15. For a methodical summary cf. ref. 7).

16. Most favorable method: Ogura, K.; Yahata, N.; Watanabe, J.; Takahashi, K.; Iida, H. Bull. Chem. Soc. Jpn. 1983, 56, 3543 .

17. a) Bordwell, F.G.; Jarvis, B.B. J. Org. Chem. 1968, 33, 1182; b) Robson, P.; Speakman, P.R.H.; Stewart, D.G. J. Chem. Soc. C 1968, 2180; c) Schank, K.; Schmitt, H.-G. unpublished results; Schmitt, H.-G.

Ph. D. Dissertation, Saarbrücken 1977; d) Kay, D.G.; Langler, R.F.; Trenholm, J.E. Can. J. Chem. 1979, $57,2185$.

18. Schank, K.; Bügler, S. presented at ISOCS 12, Nijmegen - The Netherlands 1986.

19. Philbin, E.M.; Stuart, E.R.; Timoney, R.F.; Wheeler, T.S. J. Chem. Soc. 1957, 2338.

20. Schank, K.; Bügler, S. Sulfur Lett. 1982, 1, 63; cf. Russell, G.A.; Sabourin, E.T. J. Org. Chem. 1969, 34, 2336.

21. a) Jager, J. Ph. D. Dissertation, Saarbrücken 1980; b) Schank, K.; Bügler, S. unpublished results; Bügler, S. Ph. D. Dissertation, Saarbrücken 1983; c) Ogura, K.; Kiuchi, S.; Takahashi, K.; Iida, H. Synthesis 1985, 524; d) for an independent synthesis from $\alpha$-diazomethylsulfones and sulfenyl chlorides see: Strating, J.; Reitsema, J. Rec. Trav. Chim. Pays-Bas 1966, 85, 421.

22. Preparation of $\mathbf{1 4}$ according to an olefination method published earlier by us: a) Ferdinand, G.; Schank, K.; Weber, A. Liebigs Ann. Chem. 1975, 1484; b) Ferdinand, G.; Schank, K. Synthesis 1976, 408; c) cf. ref. 21b).

23. a) Saunders, W.H., Jr.; Cockerill A.F. in Mechanisms of elimination reactions, p. 570, J. Wiley, N.Y. 1973; b) Christol, S.J.; Harrington, J.K.; Singer, M.S. J. Am. Chem. Soc. 1966, 88, 1529; c) Similar oberservations have been described with structurally related cyclopropanes: Bailey, P.L.; Hewkin, C.T.; Clegg, W.; Jackson, R.F.W. J. Chem. Soc. Perkin Trans. I 1993, 577.

24. a) Schank, K.; Schroeder, F.; Weber, A. Liebigs Ann. Chem. 1973, 553; b) Schank, K.; Schroeder, F. Liebigs Ann. Chem. 1977, 1676; c) cf. ref. 6); d) cf. ref. 1).

25. cf. Krauch, H.;Kunz W. in Reaktionen der Organischen Chemie, 5. Aufl., S. 356, Hüthig, Heidelberg 1976: a) Stuffer, E. Chem. Ber. 1890, 23, 3226; b) Backer, H.J. Rec. Trav. Chim. Bays-Bas 1951, $70,92$.

26. Gehlhaus, J.; Hoffmann, R.W. Tetrahedron 1970, 26, 5901.

27. a) Nozaki, H.; Noyori, R.; Sisido, K. Tetrahedron 1964, 20, 1125; b) Wilt, J.W.; Schneider, E.; Dabek, Jr.,H.F.; Kraemer, J.F.; Wagner, W.J. J. Org. Chem. 1966, 31, 1543.

28. Hoppmann, A.; Weyerstahl, P.; Zummack, W. Liebigs Ann. Chem. 1977, 1547.

29. Gibson, D.T. J. Chem. Soc. 1930, 12.

30. Bennett, G.M.; Statham, F.S. J. Chem. Soc. 1931, 1684.

30a. Morrill T.C. in Lanthanide Shift Reagents in Stereochemical Analysis, Methods in Stereochemical Analysis Vol. 5 (ed. A.P. Marchand), VCH Publishers Inc. 1986.

31. Schubert, H.H.; Stang, P.J. J. Org. Chem. 1984, 49, 5087.

32. a) Schroth, W.; Schädler, K.-D.; Kemmler, R. Z. Chem. 1985, 25, 170;

33. Radeglia, R.; Poleschner, H.; Schroth, W. J. Prakt. Chem. 1985, 327, 523.

34. cf. Davis, F.A.; Rizvi, S.Q.A.; Ardecky, R.; Gosciniak, D.J.; Friedman, A.J.; Yocklovich, S.G. J. Org. Chem. 1980, 45, 1650.

35. Walisch, W.; Eberle, H.G. Microchim. Acta 1967, 1031.

36. According to Walisch, W. Chem. Ber. 1961, 94, 2314.

37. Troeger, J.; Beck, O. J. Prakt. Chem. 1913, [2] 87, 289.

38. Troeger, J.;Müller, W. Arch. Pharm. 1914, 252, 39.

39. Commercially available.

40. Nakayama, J. Synthesis 1975, 38: Synthesis of 2-iso-amyloxy-benzodithiol which was reduced with DIBAL under usual conditions to 1.3 -benzodithiol in $78 \%$ yield; colorless oil, identified via oxidation.

41. Carlson, R.M.; Helquist, P.M. J. Org. Chem. 1968, 33, 2586.

42. Auret, B.J.; Boyd, D.R.; Breen, F.; Greene, R.M.E.; Robinson, P.M. J. Chem. Soc., Perkin Trans. I 1981, 930. 
43. Bordwell, F.G.; Cooper, G.D. J. Am. Chem. Soc. 1951, 73, 5184.

44. a) Hardstaff, W.R.; Langler, R.F.; Leahy, J.; Newman, M.J. Can. J. Chem. 1975, 53, 2664; b) Phillips, W.G.; Ratts, K.W. J. Org. Chem. 1971, 36, 3145; c) Goralski, C.T.; Klingler, T.C. J. Chem. Engng. Data 1976, 21, 237.

45. Bordwell, F.G.; Clemens, A.H.; Smith, D.E. J. Org. Chem. 1985, 50, 155.

46. Müller, W.; Schank, K. Chem. Ber. 1978, 111, 2870.

47. Veenstra, G.E.; Zwanenburg, B. Recl. Trav. Chim. Pays-Bas 1976, 95, 28.

48. Values from ref. 21a), recorded by a Varian A60.

49. Ferdinand, G. Ph. D. Dissertation, Saarbrücken 1976, p. 94, 118.

50. For mechanistic details cf.: Ferdinand, G.; Schank, K.; Weber, A. Liebigs Ann. Chem. 1975, 1484.

51. Values from ref. 49, recorded by a Varian A60.

52. Organikum, 15. Aufl. VEB Deutscher Verlag der Wissenschaften, Berlin 1976.

53. Beyer, H.; Bulka, E.; Beckhaus, F.-W. Chem. Ber. 1959, 92, 2593.

54. Sandstöm, J. Ark. Kemi 1955, 8, 523.

55. Used numbering for ${ }^{13} \mathrm{C}$-assignment from synonymous 6-methylthio-6-phenylsulfonyl-2- oxabicyclo[3.1.0] hexane.

56. Equal to 6-(4-methylphenylsulfonyl)-6-(4-methylphenylthio)-2-oxabicyclo[4.1.0] heptane.

57. Equal to 6-(4-methylphenylsulfonyl)-6-methylthio-2.4-dioxabicyclo[3.1.0] hexane.

58. Truce, W.E.; Mc Manimie, R.R. J. Am. Chem. Soc. 1954, 76, 5745.

* footnote: The atomic co-ordinates for this work are available on request from the Director of the Cambridge Crystallographic Data Centre, University Chemical Laboratory, Lensfield Road, Cambridge CB2 1EW. Any request should be accompanied by the full Literature citation for this communication. 